\title{
Pseudospectrum and Black Hole Quasinormal Mode Instability
}

\author{
José Luis Jaramillo®, ${ }^{1}$ Rodrigo Panosso Macedo๑, ${ }^{2}$ and Lamis Al Sheikh $\oplus^{1}$ \\ ${ }^{1}$ Institut de Mathématiques de Bourgogne (IMB), UMR 5584, CNRS, \\ Université de Bourgogne Franche-Comté, F-21000 Dijon, France \\ ${ }^{2}$ School of Mathematical Sciences, Queen Mary, University of London, \\ Mile End Road, London E1 4NS, United Kingdom
}

(Received 14 April 2020; accepted 3 May 2021; published 6 July 2021)

\begin{abstract}
We study the stability of quasinormal modes (QNM) in asymptotically flat black hole spacetimes by means of a pseudospectrum analysis. The construction of the Schwarzschild QNM pseudospectrum reveals the following: (i) the stability of the slowest-decaying QNM under perturbations respecting the asymptotic structure, reassessing the instability of the fundamental QNM discussed by Nollert [H. P. Nollert, About the Significance of Quasinormal Modes of Black Holes, Phys. Rev. D 53, 4397 (1996)] as an "infrared" effect; (ii) the instability of all overtones under small-scale ("ultraviolet") perturbations of sufficiently high frequency, which migrate towards universal QNM branches along pseudospectra boundaries, shedding light on Nollert's pioneer work and Nollert and Price's analysis [H. P. Nollert and R. H. Price, Quantifying Excitations of Quasinormal Mode Systems, J. Math. Phys. (N.Y.) 40, 980 (1999)]. Methodologically, a compactified hyperboloidal approach to QNMs is adopted to cast QNMs in terms of the spectral problem of a non-self-adjoint operator. In this setting, spectral (in)stability is naturally addressed through the pseudospectrum notion that we construct numerically via Chebyshev spectral methods and foster in gravitational physics. After illustrating the approach with the Pöschl-Teller potential, we address the Schwarzschild black hole case, where QNM (in)stabilities are physically relevant in the context of black hole spectroscopy in gravitational-wave physics and, conceivably, as probes into fundamental highfrequency spacetime fluctuations at the Planck scale.
\end{abstract}

DOI: 10.1103/PhysRevX.11.031003

\section{INTRODUCTION: QNMS AND (IN)STABILITY}

\section{A. Black hole QNM stability problem and the pseudospectrum}

Structural stability is essential in the modeling and understanding of physical phenomena. In the context of spectral problems pervading physics, often related to wave phenomena in both classical and quantum theories, this concerns, in particular, the basic question about the stability of the physical spectrum of the system. Thereupon, one needs to assess the following questions: How does the spectrum react to small changes of the underlying system? Is the spectrum stable; i.e., do small perturbations lead to tiny deviations? Or is it unstable, with small changes in the system leading to drastic modifications of the spectrum? In the present work, we study such spectral stability questions

Published by the American Physical Society under the terms of the Creative Commons Attribution 4.0 International license. Further distribution of this work must maintain attribution to the author(s) and the published article's title, journal citation, and DOI.
Subject Areas: Astrophysics, Gravitation, Interdisciplinary Physics, Optics in the setting of black hole (BH) spacetimes. Specifically, the problem we address here is the spectral robustness of BH QNMs, namely, the stability of the resonant frequencies of BHs under perturbations. From a methodological perspective, our spectral (in)stability analysis is built upon the notion of the so-called pseudospectrum.

\section{Spectral instability and pseudospectrum}

The physical status of spectral stability depends crucially on whether the underlying system is conservative or not. In particular, conservative systems have stable spectra, and therefore, the spectral instability question, being solved from scratch, is not relevant. Such spectral stability is familiar in (standard) quantum mechanics, where (timeindependent) perturbation theory precisely relies on it. It is the self-adjoint nature of the relevant operators (namely, "Hermitian matrices" in the finite-dimensional case) that accounts for such spectral stability. More systematically, it is a consequence of the so-called "spectral theorem" for self-adjoint operators: Eigenvectors form an orthogonal and complete set, whereas eigenvalues are real and stable. This theorem provides the mathematical background for the key 
physical notion of normal mode, associated with the characteristic (real) vibrating frequencies of a conservative system and its natural oscillation modes.

The situation is more delicate for nonconservative systems, modeled in terms of non-self-adjoint operators (non-Hermitian matrices). Such systems occur naturally whenever there exist flows (e.g., energy, particle, information) into external degrees of freedom that are out of the (Hilbert) space under consideration (see Ref. [1] for a recent and extensive review on non-Hermitian physics; cf. e.g., Table 1 in that work for a list of several classical and quantum systems described by non-Hermitian operators). In this setting, the spectral theorem is lost: Eigenvectors are, in general, neither complete nor orthogonal, and eigenvalues (now, in general, complex) are potentially unstable. Here, we focus on this latter point, namely, the potential spectral instability of a class of nonself-adjoint operators associated with the nonconservative system defined by the scattering of fields by BHs where, critically, the field leaks away from the system at far distances and through the $\mathrm{BH}$ horizon.

The notion of pseudospectrum [2-10] provides a powerful tool for the analysis of the properties of non-self-adjoint operators. In particular, its use is widespread whenever stability issues of nonconservative systems are addressed, from pioneering applications in hydrodynamics [2] to recent advances [11] covering a wide range in physics. Broadly speaking, in order to gain some first intuition, the pseudospectrum provides a visualization (and actually a characterization) of the spectral instability of our operator in terms of a kind of topographic map on the complex plane, where the peaks (actually end points of infinitely high throats) are at the complex eigenvalues of the nonperturbed operator. With this picture in mind, spectral stability is assessed in terms of the "thickness" of the throats: Very thin throats quickly decreasing from the eigenvalues correspond to spectral stability, whereas broad, slowly decreasing throats indicate spectral instability. In terms of level sets, contour lines corresponding to heights $1 / \epsilon$ define a family of nested sets around eigenvalues, referred to as $\epsilon$-pseudospectra, which determine the regions in which eigenvalues can potentially "migrate" under a system perturbation of size $\epsilon$. The (nonperturbed) spectrum corresponds to the set defined by $\epsilon \rightarrow 0$. Therefore, tightly packed contour lines around eigenvalues corresponding to strong gradients indicate spectral stability, whereas contour lines with low gradients extending far from the eigenvalues signal spectral instability.

\section{Black hole QNMs in gravitational physics}

$\mathrm{BH}$ QNMs encode the resonant response to (linear) perturbations of the $\mathrm{BH}$ spacetime. In spite of being triggered by perturbations, QNMs constitute an intrinsic property of the background, and therefore, QNM frequencies encode crucial geometric information about BHs and their environment. Thus, they have become a fundamental tool in astrophysics, fundamental gravitational physics, and mathematical relativity in their attempts to probe spacetime geometry through perturbation theory and scattering methods (see, e.g., Refs. [12-16] for systematic presentations and reviews).

Upon perturbation, and after an initial transient, the perturbative field propagating on the background spacetime shows an exponentially damped, oscillatory behavior. QNM frequencies are the set of complex numbers encoding the oscillatory frequencies and decaying timescales of the propagating linear (scattered) field. To fix ideas, this picture is illustrated by the $\mathrm{BH}$ formed after the merger of a compact binary, in the emerging setting of gravitationalwave $(\mathrm{GW})$ astronomy. After the transient merger phase, the resulting perturbed $\mathrm{BH}$ evolves towards stationarity in a linear ringdown phase dominated by QNMs. In particular, the late-time behavior of the GW signal is controlled by the fundamental or slowest-decaying QNM mode, namely, the QNM frequency with the smallest (in absolute value) imaginary part, which is, therefore, closest to the real axis. Nonetheless, QNMs with larger imaginary parts, referred to as overtones-with different oscillatory frequencies and faster decaying time scales than the fundamental QNM - are also present in the GW signal, its analysis being at the basis of the BH spectroscopy research program [17-23]. Beyond GW physics, QNMs play a key role in gravitational physics as a crossroads among different limits and regimes of the theory, encompassing problems in the referred GW astrophysical setting, in semiclassical gravity (e.g., Ref. [24]) and gravity-fluid (AdS/CFT) dualities (e.g., Ref. [25]), in analogue gravity [26] or in foundational questions in mathematical relativity (e.g., Refs. [27-29]), among other problems ranging from the classical to the quantum regime. In this latter setting, and given the importance they will have in our present discussion, it is worthwhile to signal that QNM overtones have been proposed as a possible probe into the quantum aspects of spacetime [16,30-43].

To be more specific, the discussion of BH QNMs is set in terms of the wave equations arising when general relativity is considered at linear order for fields propagating over a fixed $\mathrm{BH}$ background. In this work, we focus on the asymptotically flat case, where geometry approaches Minkowski spacetime (in an appropriate sense) at large distances. One must then impose appropriate boundary conditions on the underlying wave equations: As one moves far away from the source, the waves must propagate out to infinity, whereas they must propagate to the interior of the black hole at the horizon. The resulting outgoing boundary conditions define a leaky system. QNMs are obtained from the spectral problem associated with this system. A crucial point in the present discussion is that such a spectral problem can be cast as a proper "eigenvalue problem" for a certain non-self-adjoint operator. At this point, we make contact with the potential spectral 
instability that we have discussed above, associated with non-self-adjoint operators. The ultimate significance of QNM frequencies depends directly on the understanding and control of their spectral stability.

\section{B. BH QNM instability: Nollert and Price's pioneering work}

Nollert's groundbreaking work on BH QNM spectral stability [44], complemented by the analysis in Nollert and Price's work [45], shows evidence of an overall instability of the Schwarzschild QNM spectrum-affecting both the fundamental QNM and the overtones-under a class of small-scale perturbations (see also the recent works Refs. [46,47]). The analysis by Nollert and Price, both numerical and analytic in an asymptotic treatment, demonstrates the robustness of such QNM instability. However, it remains to be elucidated if such an instability corresponds to the specific form of the considered perturbations and, therefore, whether it could be an artifact of the employed approach or if it, rather, responds to a structural feature within the theory with actual implications on the physics of BH QNMs.

More specifically, these works consider a family of steplike approximations to the Schwarzschild curvature potential. In a first step, the authors calculated the QNMs corresponding to the steplike approximation for the potential (perturbed QNMs), finding a strong deviation from the original values (nonperturbed QNMs), with a clear and systematic pattern: Perturbed QNMs distribute along new QNM branches with a qualitative structure dramatically distinct from that of nonperturbed QNMs. In a second step, they performed time evolutions of the wave equation under the steplike approximated potential in a bid to identify and extract the perturbed QNMs from the wave signal. In contrast to the spectral problem, time evolutions presented an overall stable behavior under perturbations of the potential. Specifically, Nollert and Price's work demonstrates the following for the class of perturbations studied:

(i) QNM overtones are strongly unstable, with their instability increasing with their damping.

(ii) The fundamental, slowest-decaying QNM is unstable.

(iii) The black hole rings down, at intermediate late times, according to the nonperturbed fundamental mode. Only at very late times is the ringdown frequency controlled by the perturbed fundamental QNM mode.

These results have been confirmed and expanded in Refs. [46,47] to perturbations of the scattering potential, extending the steplike approximation but still sharing the feature of presenting a discontinuity at the potential or some of its derivatives.

Beyond Nollert and Price's works, research in BH QNM spectral (in)stability has been further pursued in different gravitational physics settings. In astrophysics, the understanding of possible environmental observational signatures in "dirty" BH scenarios has prompted a research line $[48,49]$ that has been significantly intensified recently [46,47, 50-52]. On the other hand, regarding investigations on the fundamental structure of spacetime, the perspective of accessing quantum scales through high-frequency instabilities of QNM overtones has also brought about systematic research [16,30-43].

In spite of these efforts, a comprehensive picture of $\mathrm{BH}$ QNM (in)stability seems to be lacking. At this point, it is worthwhile to explicitly distinguish between the instability in QNM frequencies and the instability in late ringdown frequencies. The former refers to the spectral instability in the "frequency domain" approach, when solving the spectral problem associated with the wave equation. The latter refers to a dynamical instability in the "time domain" approach, when solving the initial data dynamical problem. Both problems are intimately related but are indeed different. In particular, it is known that the two sets of frequencies can indeed decouple (e.g., Refs. [44-47,53-56] in the gravitational context). Still, the separation between QNM and ringdown frequencies signals an "anomaly" and, therefore, pinpoints a structural feature in the physical system, requiring a specific study. In the present work, we focus on QNM instability in the spectral sense.

In this context, the stability status of the slowestdecaying QNM-presenting precisely the tension described above between calculated spectral instability and observed robustness in the ringdown signal-remains unclear, whereas the elucidation of the lowest overtone subject to high-frequency instability is an open problem. In light of the discussion above on the fundamental role of $\mathrm{BH}$ QNMs in different settings of gravitational physics, the clarification of these two points is a first-order problem from a strictly physical perspective. Moreover, if establishing the stability status of BH QNMs is key in general $\mathrm{BH}$ physics, the problem is actually urgent in gravitationalwave astrophysics. Indeed, in the era of gravitational-wave astronomy, the stability of the fundamental QNM and the overtones is paramount for $\mathrm{BH}$ spectroscopy.

The implementation of an analysis based on the pseudospectrum allows us to address these questions systematically and to provide sound answers to points (i) and (ii) above. In short, and anticipating the results discussed later in detail, such an analysis confirms the instability behavior of QNM overtones-point (i) - and provides a framework for its systematic study, whereas it disproves the instability of the fundamental QNM - point (ii) - if asymptotic properties of the spacetime are respected, with its unstable behavior in Ref. [44] resulting in an artifact as a consequence of "cutting" the effective potential at a finite distance. Regarding point (iii), from the stability of the fundamental QNM, we conclude that the late-time ringdown is indeed dominated by the unperturbed slowest-decaying QNM (without any very late transition to a "perturbed ringdown" frequency), but the systematic analysis of the detailed relation between QNM frequencies and $\mathrm{BH}$ ringdown frequencies is beyond the 
scope of the present work and will be the subject of a specifically targeted research focused on the potential implications of $\mathrm{BH}$ QNM instability on GW astrophysics [57].

\section{Present approach}

\section{Basic ingredients: Hyperboloidal approach and pseudospectrum}

The calculation of BH QNMs has been the subject of systematic study in gravitational physics, and there exists a variety of standard approaches to address this problem (cf. e.g., Refs. [13-16]). From a methodological perspective, our discussion relies on two main ingredients at a conceptual level:

(i) A hyperboloidal approach to QNMs: This geometric approach casts the QNM calculation as a proper eigenvalue problem of a particular non-self-adjoint operator.

(ii) Pseudospectrum: Together with related spectral tools, this notion provides the key instrument to study the potential spectral instability of the relevant non-self-adjoint operator.

The combination of these two elements allows us to develop a systematic treatment of the problem. To the best of our knowledge, no systematic treatment of BH QNM (in) stability based on the pseudospectrum exists in the literature. At a first exploratory stage, prior to a full analytical study, the present work addresses pseudospectra using a numerical approach. This sets a challenging numerical problem demanding high accuracy, which is addressed here by introducing a third key ingredient in our approach: the use of spectral numerical methods.

\section{Beyond gravity: QNMs, pseudospectrum, and interdisciplinary physics}

Before entering into the detailed discussion of BH QNM stability, let us stress that both QNMs and the pseudospectrum provide independent, but indeed complementary, arenas for interdisciplinary research in physics and related disciplines.

Regarding QNMs, beyond the present gravitational context, the notion of QNM spreads in physics, e.g., in electromagnetism and optics, acoustics, or-under the related notion of resonance in quantum mechanics-in atomic, nuclear, and molecular physics. Beyond physics, QNMs enter into the discussion of scattering problems in geometry [58] and chaotic dynamics (see Refs. [59,60] for a systematic review of scattering resonances or QNMs from a mathematical perspective). Together with the extent of the applicability of the QNM notion, an important aspect concerns timing. Indeed, the synergy observed in this sense in recent years among different subdisciplines in the gravitational setting (namely, GW astrophysics, AdS/ CFT dualities, and mathematical relativity) remarkably extends to other fields in physics, as perfectly illustrated by recent breakthroughs in optical nanoresonator QNMs, namely, photonic and plasmonic resonances $[61,62]$.

Regarding the pseudospectrum, its use in physics naturally occurs in the study of stability and spectral problems in nonconservative systems, from which we highlight its applications in hydrodynamics [2] and in non-Hermitian quantum mechanics [8]. Beyond physics, systematic applications are found in numerical analysis, the original context where the notion was formulated. This wide range of applications becomes intertwined methodologically by the pseudospectrum. The present approach to $\mathrm{BH}$ QNM stability, which introduces (to the best of our knowledge) the pseudospectrum to gravity, incorporates gravitational physics into this multifaceted research scheme. When combined with the large range of applicability of the QNM notion in physics, it outlines a robust and potentially rich frame for interdisciplinary research in physics. The last section of this article, Sec. VII B 4, expands on this perspective.

The article is structured as follows. Section II presents a qualitative description of the hyperboloidal approach to scattering problems and reviews the literature on this geometrical framework. Beyond reviewing the main concepts, with a focus on QNMs, this section identifies and constructs the appropriate scalar product in the problem. Section III introduces the basic elements to study spectral instability of non-self-adjoint operators, in particular, the notion of pseudospectrum. Section IV presents the numerical spectral tools that will be employed in the present approach. Then, Sec. V illustrates all the previous elements in the toy model provided by the Pöschl-Teller potential, which also anticipates some of the main results in the $\mathrm{BH}$ setting. Section VI contains the main contribution in the present work, namely, the construction of the Schwarzschild QNM pseudospectrum and the consequent analysis of BH QNM (in)stability. Finally, conclusions and perspectives are presented in Sec. VII. Four Appendixes complete some points in the technical discussion of the main text. Throughout this work, we adopt units in which the speed of light and the gravitational constant are $c=G=1$.

\section{HYPERBOLOIDAL APPROACH TO QNMS}

\section{A. Hyperboloidal approach: Heuristic introduction}

Our approach to QNMs strongly relies on casting the discussion in terms of the spectral problem of a (non-selfadjoint) operator. In our scheme, this is achieved by means of a so-called hyperboloidal approach to wave propagation, which provides a systematic framework exploiting the geometric asymptotics of the spacetime, in particular, enforcing the relevant outgoing boundary conditions in a geometric way. We start with a heuristic discussion of the basics, aiming at providing an intuitive picture and explicitly sacrificing rigor. 
The notion of wave zone is a familiar concept in physics. It describes a region far away from a source where the degrees of freedom of a given field (not necessarily linear) propagate as a free wave, independently of their interior sources and obeying the superposition principle. Roughly speaking, this region is characterized by $r / R \gg 1$, where $r$ is the location of a distant observer and $R$ is a typical length scale of the source. This concept is addressed formally by taking the appropriate limit $r \rightarrow \infty$ or $1 / r \rightarrow 0$. From a spacetime perspective, however, such a limit must be carefully understood.

To fix ideas, let us consider a physical scenario in spherical symmetry, where a wave propagating at finite speed is described in a standard spherical coordinate system $(t, r, \theta, \varphi)$ (for simplicity, let us momentarily consider a flat spacetime where we ignore the effects of gravity). The retarded time coordinate $u=t-r$ corresponds to the time at which an outgoing wave, passing by the observer at $r$ at time $t$, was emitted by a source located at the origin. Crucially, "light rays" propagate along (characteristic) curves satisfying $u=$ const. In this setting, and as illustrated in Fig. 1, taking the limit $r \rightarrow \infty$ corresponds to completely different geometric statements depending on whether one stays at the hypersurface $t=$ const or rather on $u=$ const. The limit attained by "spacelike" (geodesic) curves satisfying the

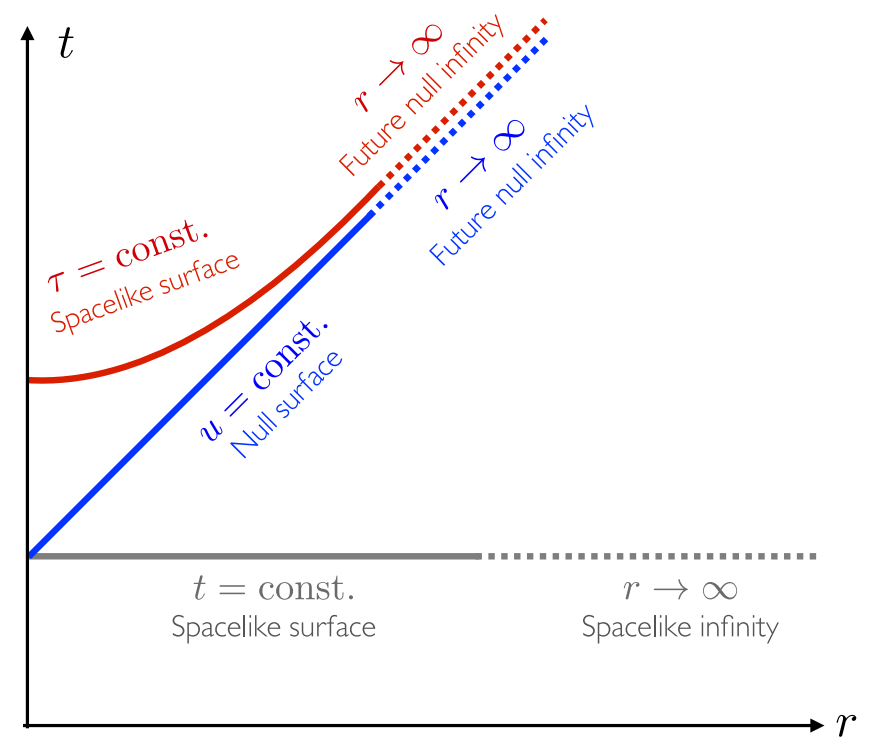

FIG. 1. Schematic representation of the different " $r \rightarrow \infty$ " limits along curves within different types of spacetime hypersurfaces. Cauchy hypersurfaces, of spacelike character and represented by the " $t=$ const" condition in the figure, are such that this limit attains the so-called spatial infinity $i^{0}$, whereas in null hypersurfaces satisfying " $u=$ const" (with $u=t-r$ a null "retarded time") the limit attains the outgoing wave zone modeled by future null infinity $\mathcal{I}^{+}$. The hyperboloidal approach offers an intermediate possibility, where the limit is taken along spacelike hypersurfaces, formally represented by the " $\tau=$ const" but still reaching $\mathcal{I}^{+}$asymptotics. former condition $(t=$ const) is referred to as "spacelike infinity" and denoted $i^{0}$, whereas lightlike or null (future geodesic) curves satisfying the latter condition $(u=$ const) attain a limit referred to as future null infinity, denoted as $\mathcal{I}^{+}$. It is future null infinity $\mathcal{I}^{+}$that formally captures the intuitive notion of outgoing wave zone.

Other alternatives to the $t=$ const and $u=$ const hypersurfaces are possible, which is natural in a general relativistic context implementing freedom of coordinate choice. A particularly convenient possibility in our present problem consists in choosing a third alternative: to keep spacelike hypersurfaces defined as level sets of an appropriate time function $\tau$ while reaching future null infinity as $r \rightarrow \infty$ so as to enforce the outgoing character of the radiation. Such a third option is displayed in Fig. 1 as a $\tau=$ const hypersurface. The asymptotic geometry of such hypersurfaces is that of a hyperboloid, a feature that gives the resulting hyperboloidal approach its name.

The previous heuristic picture of spacetime asymptotics is formalized in the geometric notion of conformal infinity [63-69], which provides a rigorous and geometrically welldefined strategy to deal with radiation problems of compact isolated bodies. A conformal compactification maps the infinities of the physical spacetime into a finite region delimited by the boundaries of a conformal manifold. Specifically, $\mathcal{I}^{+}$corresponds to the future endpoints of null geodesics, whereas a time function $\tau$ will be referred to as hyperboloidal if hypersurfaces $\tau=$ const intersect $\mathcal{I}^{+}$, therefore being adapted to the geometrical structure at the infinitely far-away wave zone.

The hyperboloidal formulation has proved to be a powerful tool in mathematical and numerical relativity, allowing to obtain existence results in the nonlinear treatment of Einstein equations, as illustrated in the semiglobal result in Ref. [70], or providing a natural framework for the extraction of the GW waveform in numerical dynamical evolutions of GW sources. Together with those fully nonlinear studies, over the last decade, the hyperboloidal approach has been successfully applied to problems defined on fixed spacetime backgrounds (see, e.g., Ref. [71] and references therein). In particular, Ref. [72] proposed a hyperboloidal approach to $\mathrm{BH}$ perturbation theory.

This is our setting for QNMs, where the hyperboloidal framework allows us to geometrically implement the outgoing boundary conditions at $\mathcal{I}^{+}$, in a strategy first proposed by Schmidt in Ref. [73]. The adopted (compactified) hyperboloidal approach provides a geometric framework to study QNMs that characterizes resonant frequencies in terms of an eigenvalue problem [28,71-81]. As explained above, the scheme geometrically imposes QNM outgoing boundary conditions by adopting a spacetime slicing that intersects future null infinity $\mathcal{I}^{+}$and, in the BH setting, penetrates the horizon. Since light cones point outwards at the boundary of the domain, outgoing boundary conditions are automatically 
imposed for propagating physical degrees of freedom. Along these lines, our scheme to address the BH QNM (in)stability problem strongly relies on the hyperboloidal approach since it provides the rationale to define the nonself-adjoint operator on which a pseudospectrum analysis is then performed.

\section{B. Wave equation in the compactified hyperboloidal approach}

We focus on the propagation and, more generally, the scattering problem of (massless) linear fields on stationary, spherically symmetric, BH backgrounds. For concreteness, let us first consider a scalar field $\Phi$, satisfying the wave equation

$$
\square \Phi=\nabla^{a} \nabla_{a} \Phi=0 .
$$

We adopt standard Schwarzschild coordinates

$$
d s^{2}=-f(r) d t^{2}+f(r)^{-1} d r^{2}+r^{2}\left(d \theta^{2}+\sin ^{2} \theta d \varphi^{2}\right)
$$

and emphasize that $t=$ const slices correspond to Cauchy hypersurfaces intersecting both the horizon bifurcation sphere and spatial infinity $i^{0}$. If we consider the rescaling

$$
\Phi=\frac{1}{r} \phi,
$$

then Eq. (1) is rewritten, expanding $\phi$ in spherical harmonics with $\phi_{\ell m}$ modes and using the tortoise coordinate defined by $\left(d r / d r_{*}\right)=f(r)$ (with the appropriate integration constant), as

$$
\left(\frac{\partial^{2}}{\partial t^{2}}-\frac{\partial^{2}}{\partial r_{*}^{2}}+V_{\ell}\right) \phi_{\ell m}=0
$$

where now $\left.r_{*} \in\right]-\infty, \infty[$. Remarkably, when considering electromagnetic and (linearized) gravitational fields, the respective geometric wave equations corresponding to Eq. (1) can be cast in the form of Eq. (4) for appropriate effective scalar potentials. Specifically, two scalar fields with different parity can be introduced, satisfying Eq. (4) with suitable potentials $V_{\ell}$. In the gravitational case, the axial parity is subject to the so-called Regge-Wheeler potential, whereas the polar one is controlled by the Zerilli potential (cf. e.g., Refs. [12,13,82]).

The $\mathrm{BH}$ event horizon and (spatial) infinity correspond, respectively, to $r_{*} \rightarrow-\infty$ and $r_{*} \rightarrow+\infty$. We extend the domain of $r_{*}$ to $[-\infty, \infty]$ and introduce the dimensionless quantities

$$
\bar{t}=\frac{t}{\lambda}, \quad \bar{x}=\frac{r_{*}}{\lambda}, \quad \hat{V}_{\ell}=\lambda^{2} V_{\ell},
$$

for an appropriate length scale $\lambda$ to be chosen in each specific setting. More importantly, we consider coordinates $(\tau, x)$ that implement the compactified hyperboloidal approach

$$
\left\{\begin{array}{l}
\bar{t}=\tau-h(x) \\
\bar{x}=g(x)
\end{array}\right.
$$

Specifically (see Fig. 2), we consider the following:

(i) The height function $h(x)$ implements the hyperboloidal slicing; i.e., $\tau=$ const is a horizon-penetrating hyperboloidal slice $\Sigma_{\tau}$ intersecting future $\mathcal{I}^{+}$.

(ii) The function $g(x)$ introduces a spatial compactification from $\bar{x} \in[-\infty, \infty]$ to a compact interval $[a, b]$.

We note that the compactification is performed only in the spatial direction along the hyperboloidal slice, and not in time, so the latter can be Fourier transformed in an unbounded domain. The relevant compactification here is a partial one and not the total spacetime compactification leading to Carter-Penrose diagrams. The choice of $h(x)$ and $g(x)$ is, as we comment below, subject to certain restrictions. Under transformation (6), the wave equation (4) is written

$$
\begin{aligned}
& {\left[\left(1-\left(\frac{h^{\prime}}{g^{\prime}}\right)^{2}\right) \partial_{\tau}^{2}-\frac{2}{g^{\prime}}\left(\frac{h^{\prime}}{g^{\prime}}\right) \partial_{\tau} \partial_{x}-\frac{1}{g^{\prime}}\left(\frac{h^{\prime}}{g^{\prime}}\right)^{\prime} \partial_{\tau}\right.} \\
& \left.-\frac{1}{g^{\prime}} \partial_{x}\left(\frac{1}{g^{\prime}} \partial_{x}\right)+\hat{V}_{\ell}\right] \phi_{\ell m}=0,
\end{aligned}
$$

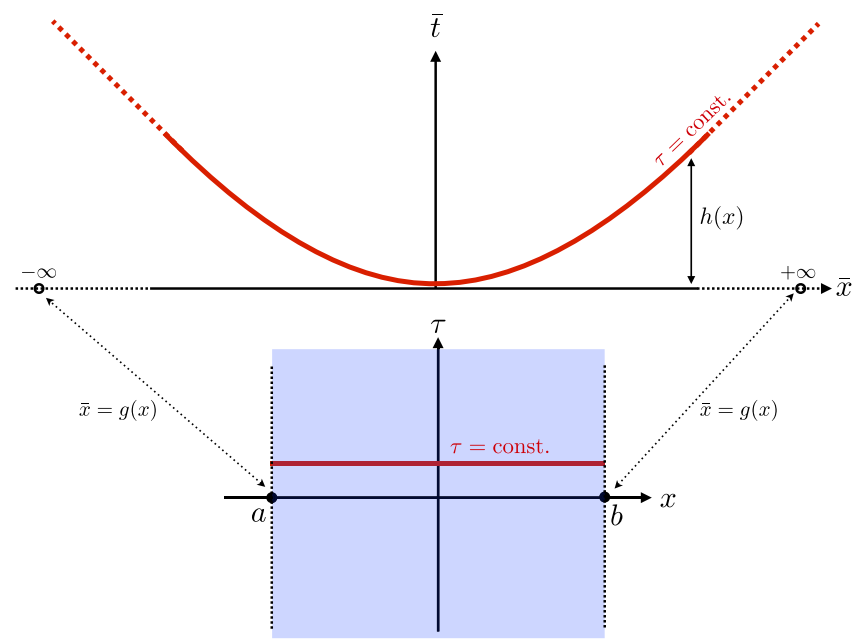

FIG. 2. Schematic representation of the hyperboloidal coordinate transformation in Eq. (6). Top panel: Dimensionless Schwarzschild time and tortoise coordinates $(\bar{t}, \bar{x})$. The height function $h(x)$ bends the time slices so that future null infinity (and/or the black hole horizon) is reached in the limit $\bar{x} \rightarrow \pm \infty$. Bottom panel: Spatially compactified hyperboloidal coordinates $(\tau, x)$. The compactification function $g(x)$ maps the infinite domain $\bar{x} \in]-\infty, \infty[$ onto the finite interval $x \in] a, b[$. Points $b$ and $a$ are added at the boundary, representing null infinity $\mathcal{I}^{+}$ and/or the $\mathrm{BH}$ horizon. The blue stripe shows the domain of integration of the wave equation in Eq. (7) in these compactified hyperboloidal coordinates, namely, $(\tau, x) \in]-\infty,+\infty[\times[a, b]$, corresponding to the full original domain $(\bar{t}, \bar{x}) \in \mathbb{R}^{2}$ of Eq. (4). 
where the prime denotes the derivative with respect to $x$. Admittedly, expression (7) appears to be more intricate than Eq. (4). However, this change encodes a neat geometric structure, and, as we shall argue, it plays a crucial role in our construction and discussion of the relevant spectral problem.

\section{First-order reduction in time and spectral problem}

The structure in Eq. (7) is made more apparent by performing a first-order reduction in time, by introducing

$$
\psi_{\ell m}=\partial_{\tau} \phi_{\ell m}, \quad u_{\ell m}=\left(\begin{array}{c}
\phi_{\ell m} \\
\psi_{\ell m}
\end{array}\right) .
$$

Then, Eq. (7) becomes

$$
\partial_{\tau} u_{\ell m}=i L u_{\ell m}
$$

where the operator $L$ is defined as

$$
L=\frac{1}{i}\left(\begin{array}{c|c}
0 & 1 \\
\hline L_{1} & L_{2}
\end{array}\right),
$$

with

$$
\begin{aligned}
& L_{1}=\frac{1}{w(x)}\left(\partial_{x}\left(p(x) \partial_{x}\right)-q_{\ell}(x)\right), \\
& L_{2}=\frac{1}{w(x)}\left(2 \gamma(x) \partial_{x}+\partial_{x} \gamma(x)\right),
\end{aligned}
$$

and

$$
\begin{aligned}
w(x) & =\frac{g^{\prime 2}-h^{\prime 2}}{\left|g^{\prime}\right|}, \quad p(x)=\frac{1}{\left|g^{\prime}\right|}, \quad q_{\ell}(x)=\left|g^{\prime}\right| \hat{V}_{\ell}, \\
\gamma(x) & =\frac{h^{\prime}}{\left|g^{\prime}\right|} .
\end{aligned}
$$

The structure of $L_{1}$ is that of a Sturm-Liouville operator. In particular, functions $h(x)$ and $g(x)$ are chosen such that they guarantee the positivity of the weight function $w(x)$, namely, $w(x)>0$. The operator $L_{2}$ also has a neat geometric and analytic structure adapted to the integration by parts, being symmetric in the following form: $L_{2}=\frac{1}{w(x)}\left\{\gamma(x) \partial_{x}+\partial_{x}[\gamma(x) \cdot]\right\}$.

A key property of coordinate transformation (6) is that it preserves, up to the overall constant $\lambda$, the timelike Killing vector $t^{a}$ controlling stationarity,

$$
t^{a}=\partial_{t}=\frac{1}{\lambda} \partial_{\bar{t}}=\frac{1}{\lambda} \partial_{\tau}
$$

In this sense, functions $t$ and $\lambda \tau$ "tick" at the same pace; namely, they are natural parameters of $t^{a}$, i.e., $t^{a}(t)=$ $t^{a}(\lambda \tau)=1$ (the role of the constant $\lambda$ being just that of keeping proper dimensions). This property is crucial for the consistent definition of QNM frequencies by Fourier (or Laplace) transformation from Eqs. (4) and (7) since the corresponding variables $\omega$ respectively conjugate to $t$ and $\tau$ then coincide (up to the constant $1 / \lambda$ ). In other words, the change of time coordinate in Eq. (6) does not affect the values of the obtained QNM frequencies.

Then, performing the Fourier transform in $\tau$ in the firstorder (in time) form (9) of the wave equation [with standard sign convention for the Fourier modes, $u_{\ell m}(\tau, x) \sim$ $\left.u_{\ell m}(x) e^{i \omega \tau}\right]$, we arrive at the spectral problem for the operator $L$,

$$
L u_{n, \ell m}=\omega_{n, \ell m} u_{n, \ell m},
$$

or, more explicitly,

$$
\left(\begin{array}{c|c}
0 & 1 \\
\hline L_{1} & L_{2}
\end{array}\right)\left(\begin{array}{l}
\phi_{\ell m} \\
\psi_{\ell m}
\end{array}\right)=i \omega_{n, \ell m}\left(\begin{array}{c}
\phi_{n, \ell m} \\
\psi_{n, \ell m}
\end{array}\right) .
$$

\section{Regularity and outgoing boundary conditions}

As emphasized at the beginning of this section, a major motivation for the adopted hyperboloidal approach is the geometric imposition of outgoing boundary conditions at future null infinity and at the event horizon: Being null hypersurfaces with light cones pointing outwards from the integration domain, the physical, causally propagating degrees of freedom (such as the scalar fields we consider here) should not admit boundary conditions, as long as they satisfy the appropriate regularity conditions. How does this translate into the analytic scheme resulting from the change of variables in Eq. (6)?

The key point is that transformation (6) must be such that $p(x)$ in the Sturm-Liouville operator $L_{1}$ in Eq. (11) vanishes at the boundaries of the compactified spatial domain $[a, b]$,

$$
p(a)=p(b)=0 .
$$

This point will be illustrated explicitly in the cases discussed later. Then, the elliptic operator $L_{1}$ is a "singular" Sturm-Liouville operator, which impacts directly on the boundary conditions it admits. Specifically, if (appropriate) regularity is enforced on eigenfunctions, then $L_{1}$ does not admit boundary conditions. Moreover, such absence of boundary conditions extends to the full operator $L$ in the hyperbolic problem. In brief, if sufficient regularity is imposed on the space of functions $u_{n, \ell m}$, then wave equations (7) and (9) and the spectral problem (14) do not admit boundary conditions, as a consequence of the vanishing of $p(x)$ at the boundaries of $[a, b]$.

This feature is the analytic counterpart of the geometric structure implemented in the compactified hyperboloidal approach. QNM boundary conditions are built into the 
"bulk" of the operator $L$ as regularity conditions in Eqs. (14) and (15).

\section{Scalar product: QNMs as a non-self-adjoint spectral problem}

The outgoing boundary conditions in the present setting define a leaky system, with a loss of energy through the boundaries-null infinity and the black hole horizon-so the system is not conservative, which suggests that the infinitesimal generator of the evolution in Eq. (7), namely, the operator $L$, should be non-self-adjoint. Thus, we need to introduce an appropriate scalar product in the problem. Moreover, such identification of the appropriate Hilbert space for solutions is also key for the regularity conditions evoked above.

Equation (7) describes the evolution of each mode $\phi_{\ell m}$ in a background $1+1$-Minkowski spacetime with a scattering potential $V_{\ell}$. A natural scalar product in this reduced problem (cf. Ref. [83] for an extended discussion in terms of the full problem), both from the physical and the analytical point of view, is given in terms of the energy associated with such a scalar field mode. In the context of the spectral problem (14), we must generically consider a complex scalar field $\phi_{\ell m}$, for which the associated stressenergy tensor [dropping $(\ell, m)$ indices] is

$T_{a b}=\frac{1}{2}\left(\nabla_{a} \bar{\phi} \nabla_{b} \phi-\frac{1}{2} \eta_{a b}\left(\nabla^{c} \bar{\phi} \nabla_{c} \phi+V_{e} \bar{\phi} \phi\right)+\right.$ c.c. $)$,

where $\eta_{a b}$ denotes the Minkowski metric in arbitrary coordinates and "c.c." indicates "complex conjugate." In a stationary situation, the "total energy" contained in the spatial slice $\Sigma_{\tau}$ and associated with the mode $\phi$ is given [67] by

$$
E=\int_{\Sigma_{\tau}} T_{a b}(\phi, \nabla \phi) t^{a} n^{b} d \Sigma_{\tau}
$$

where $t^{a}$ is, again, the timelike Killing vector associated with stationarity and $n^{a}$ denotes the unit timelike normal to the spacelike slice $\Sigma_{\tau}$. Explicitly writing the (dimensionless) energy in the compactified hyperboloidal coordinates $(\tau, x)$ in Eq. (6), we get

$$
\begin{aligned}
E\left(\phi, \partial_{\tau} \phi\right)= & \int_{\Sigma_{\tau}} T_{a b}\left(\phi, \partial_{\tau} \phi\right) t^{a} n^{b} d \Sigma_{\tau} \\
= & \frac{1}{2} \int_{a}^{b}\left[\left(g^{\prime 2}-h^{\prime 2}\right) \partial_{\tau} \bar{\phi} \partial_{\tau} \phi+\partial_{x} \bar{\phi} \partial_{x} \phi+g^{\prime 2} \hat{V}_{\ell} \bar{\phi} \phi\right] \\
& \times \frac{1}{\left|g^{\prime}\right|} d x
\end{aligned}
$$

where we identify the functions appearing in the definition of the $L_{1}$ operator in Eqs. (11) and (12). In particular, if $g^{\prime 2}-h^{\prime 2}>0$ (as we have required above) and $\hat{V}_{\ell}>0$ (this is required for positivity of the norm), then, identifying $\partial_{\tau} \phi=\psi$ as in Eq. (8), we can write the following norm for the vector $u$ in Eq. (8):

$$
\begin{aligned}
\|u\|_{E}^{2} & =\left\|\left(\begin{array}{c}
\phi \\
\psi
\end{array}\right)\right\|_{E}^{2}:=E(\phi, \psi) \\
& =\frac{1}{2} \int_{a}^{b}\left(w(x)|\psi|^{2}+p(x)\left|\partial_{x} \phi\right|^{2}+q_{\ell}(x)|\phi|^{2}\right) d x .
\end{aligned}
$$

In the following, we refer to this norm as the "energy norm." We notice that $\gamma(x)$ in Eq. (12), associated with $L_{2}$, does not enter in the norm, that is, in the energy. This norm indeed comes from a scalar product. To make its role more apparent, we rewrite the $q_{\ell}(x)$ function as the rescaled potential $\tilde{V}_{\ell}$,

$$
\tilde{V}_{\ell}:=q_{\ell}(x)=\left|g^{\prime}(x)\right| \hat{V}_{\ell}=\frac{\hat{V}_{\ell}}{p(x)},
$$

and under the assumption above, $\tilde{V}_{\ell}>0$, we can introduce the "energy scalar product" for vector functions $u$ in Eq. (8) as

$$
\begin{aligned}
\left\langle u_{1}, u_{2}\right\rangle_{E}= & \left\langle\left(\begin{array}{l}
\phi_{1} \\
\psi_{1}
\end{array}\right),\left(\begin{array}{l}
\phi_{2} \\
\psi_{2}
\end{array}\right)\right\rangle_{E} \\
= & \frac{1}{2} \int_{a}^{b}\left(w(x) \bar{\psi}_{1} \psi_{2}+p(x) \partial_{x} \bar{\phi}_{1} \partial_{x} \phi_{2}\right. \\
& \left.+\tilde{V}_{\ell} \bar{\phi}_{1} \phi_{2}\right) d x .
\end{aligned}
$$

By construction, it holds that $\|u\|_{E}^{2}=\langle u, u\rangle_{E}$. This scalar product will be the relevant one in our discussion.

The full operator $L$ in Eq. (14) is not self-adjoint in the scalar product (22). In fact, the first-order operator $L_{2}$ stands for a dissipative term encoding the energy leaking at $\mathcal{I}^{+}$and the $\mathrm{BH}$ horizon [83]. At first glance, one could consider that this is related to the first-order character of $L_{2}$, which makes it antisymmetric when integrating by parts with an $L^{2}([a, b], w(x) d x)$ scalar product on $\psi$, in contrast with the self-adjoint character of the Sturm-Liouville operator $L_{1}$ in $L^{2}([a, b], w(x) d x)$ for $\phi$ functions. However, this is misleading and actually suggests a wrong bulk dissipation mechanism. When calculating the formal adjoint $L^{\dagger}$ of the full operator $L$ with the scalar product (22), one gets

$$
L^{\dagger}=L+L^{\partial},
$$

where $L^{\partial}$ is an operator with support only on the boundaries of the interval $[a, b]$, which we can formally write as

$$
L^{\partial}=\frac{1}{i}\left(\begin{array}{c|c}
0 & 0 \\
\hline 0 & L_{2}^{\partial}
\end{array}\right),
$$

with $L_{2}^{\partial}$ given by the expression 


$$
L_{2}^{\partial}=2 \frac{\gamma(x)}{w(x)}(\delta(x-a)-\delta(x-b))
$$

where $\delta(x)$ formally denotes a Dirac-delta distribution. This formal expression precisely underlines the need for a more careful treatment of the involved functional spaces, but it has the virtue of making it apparent that the obstruction to self-adjointness lies at the boundaries, as one expects in our QNM problem, and not in the bulk, as one could naively conclude from the presence of a firstorder operator $L_{2}$ (cf. discussion above): $L_{2}^{\partial}$ explicitly entails a boundary dissipation mechanism. In particular, we note that $L$ is self-adjoint in the nondissipative $L_{2}=0$ case, as expected, but this requires the introduction of a quite nontrivial scalar product.

As a bottom line, in this section we have cast the QNM problem as the eigenvalue problem of a non-self-adjoint operator. In the following section, we discuss the implications of this approach.

\section{SPECTRAL STABILITY AND PSEUDOSPECTRUM}

The spectrum of a non-self-adjoint operator is potentially unstable under small perturbations of the operator. Let us consider a linear operator $A$ on a Hilbert space with scalar product $\langle\cdot, \cdot\rangle$, and denote its adjoint by $A^{\dagger}$, satisfying $\left\langle A^{\dagger} u, v\right\rangle=\langle u, A v\rangle$. The operator $A$ is called normal if and only if $\left[A, A^{\dagger}\right]=0$. In particular, a self-adjoint operator $A^{\dagger}=A$ is normal. In this setting, the "spectral theorem" (under the appropriate functional space assumptions) states that a normal operator is characterized as being unitarily diagonalizable. The eigenfunctions of $A$ form an orthonormal basis, and crucially in the present discussion, the eigenvalues are stable under perturbations of $A$. The lack of such a spectral theorem for non-normal operators entails a severe loss of control on eigenfunction completeness and the potential instability of the spectrum of the operator $A$. Here, we focus on this second aspect.

\section{A. Spectral instability: Eigenvalue condition number}

Let us consider an operator $A$ and an eigenvalue $\lambda_{i}$. Left $w_{i}$ and right $v_{i}$ eigenvectors are characterized as [84]

$$
A^{\dagger} w_{i}=\bar{\lambda}_{i} w_{i}, \quad A v_{i}=\lambda_{i} v_{i},
$$

with $\bar{\lambda}_{i}$ the complex conjugate of $\lambda_{i}$. Let us consider, for $\epsilon>0$, the perturbation of $A$ by a (bounded) operator $\delta A$,

$$
A(\epsilon)=A+\epsilon \delta A, \quad\|\delta A\|=1 .
$$

The eigenvalues [85] in the perturbed spectral problem

$$
A(\epsilon) v_{i}(\epsilon)=\lambda_{i}(\epsilon) v_{i}(\epsilon)
$$

satisfy

$$
\begin{aligned}
\left|\lambda_{i}(\epsilon)-\lambda_{i}\right| & =\epsilon \frac{\left|\left\langle w_{i}, \delta A v_{i}(\epsilon)\right\rangle\right|}{\left|\left\langle w_{i}, v_{i}\right\rangle\right|}=\epsilon \frac{\left|\left\langle w_{i}, \delta A v_{i}\right\rangle\right|}{\left|\left\langle w_{i}, v_{i}\right\rangle\right|}+O\left(\epsilon^{2}\right) \\
& \leq \epsilon \frac{\left\|w_{i}\right\|\left\|\mid \delta A v_{i}\right\|}{\left|\left\langle w_{i}, v_{i}\right\rangle\right|}+O\left(\epsilon^{2}\right) \\
& \leq \epsilon \frac{\left\|w_{i}\right\|\left\|v_{i}\right\|}{\left|\left\langle w_{i}, v_{i}\right\rangle\right|}+O\left(\epsilon^{2}\right)
\end{aligned}
$$

where the first line generalizes $[6,86]$ the expression employed (for self-adjoint operators, where $w_{i}=v_{i}$ ) in quantum mechanics first-order perturbation theory, the first inequality in the second line is the Cauchy-Schwartz inequality, and in the second inequality we make explicit use of an operator norm $\|\cdot\|$ induced from that of the vector Hilbert space so that $\|\delta A v\| \leq\|\delta A\|\|v\|$ and $\|\delta A\|=1$ in Eq. (27). Then, defining the condition number $\kappa_{i}$ associated with the eigenvalue $\lambda_{i}$, we can write the bound for the perturbation of the eigenvalue $\lambda_{i}$,

$$
\left|\lambda_{i}(\epsilon)-\lambda_{i}\right| \leq \epsilon \kappa_{i}, \quad \kappa_{i}=\kappa\left(\lambda_{i}\right):=\frac{\left\|w_{i}\right\|\left\|v_{i}\right\|}{\left|\left\langle w_{i}, v_{i}\right\rangle\right|} .
$$

In the normal operator case, $w_{i}$ and $v_{i}$ are proportional (namely, since $A$ and $A^{\dagger}$ commute, they can be diagonalized in the same basis). Then, again by the Cauchy-Schwartz inequality, $\kappa_{i}=1$, and we encounter spectral stability: A small perturbation of order $\epsilon$ of the operator $A$ entails a perturbation of the same order $\epsilon$ in the spectrum. In contrast, in the non-normal case, $w_{i}$ and $v_{i}$ are not necessarily collinear. In the absence of a spectral theorem, nothing prevents $w_{i}$ and $v_{i}$ from becoming close to orthogonality, and $\kappa_{i}$ can become very large: Small perturbations of $A$ can produce large deviations in the eigenvalues. The relative values of $\kappa_{i}$ control the corresponding instability sensitivity of different $\lambda_{i}$ 's to an operator perturbation [87].

\section{B. Pseudospectrum}

A complementary approach to the study of the spectral (in)stability of the operator $A$ under perturbations consists in considering the following questions:

Given the operator $A$ and its spectrum $\sigma(A)$, which is the set formed by complex numbers $\lambda \in \mathbb{C}$ that are actual eigenvalues of "some" small perturbation $A+\delta A$, with $\|\delta A\|<\epsilon$ ? Does this set extend in $\mathbb{C}$ far from the spectrum of $A$ ?

In this setting, if we are dealing with an operator that is spectrally stable, we expect that the spectrum of $A+\delta A$ will not change strongly with respect to that of $A$, so the set of $\lambda \in \mathbb{C}$ corresponding to the first question above will not be far from $\sigma(A)$, staying in its vicinity at a maximum distance of order $\epsilon$. On the contrary, if we find a tiny perturbation $\delta A$ of order $\|\delta A\|<\epsilon$ such that the corresponding eigenvalues of $A+\delta A$ actually reach regions in $\mathbb{C}$ 
at distances far from $\sigma(A)$, namely, orders of magnitude above $\epsilon$, we conclude that our operator suffers from an actual spectral instability.

\section{Pseudospectrum and operator perturbations}

The previous discussion is formalized in the notion of pseudospectrum, leading to the following (first) definition [88].

Definition 1: (Pseudospectrum: perturbative approach). Given $A \in M_{n}(\mathbb{C})$ and $\epsilon>0$, the $\epsilon$-pseudospectrum $\sigma^{\epsilon}(A)$ of $A$ is

$$
\begin{aligned}
& \sigma^{\epsilon}(A) \\
& =\left\{\lambda \in \mathbb{C}, \exists \delta A \in M_{n}(\mathbb{C}),\|\delta A\|<\epsilon: \lambda \in \sigma(A+\delta A)\right\} .
\end{aligned}
$$

This notion of the $\epsilon$-pseudospectrum $\sigma^{\epsilon}(A)$ is a crucial one in our study of eigenvalue instability since it implies that points in $\sigma^{\epsilon}(A)$ are actual eigenvalues of some perturbation of $A$ of order $\epsilon$ : If $\sigma^{\epsilon}(A)$ extends far from the spectrum $\sigma(A)$ for a small $\epsilon$, then a small physical perturbation $\delta A$ of $A$ can produce large actual deviations in the perturbed physical spectrum. The pseudospectrum becomes a systematic tool to assess spectral (in)stability, as illustrated in the hydrodynamics context [2].

Although the characterization (31) of $\sigma^{\epsilon}(A)$ neatly captures the notion of (in)stability of $A$, from a pragmatic perspective it suffers from the drawback of not providing a constructive approach to build such sets $\sigma^{\epsilon}(A)$ for different $\epsilon$ 's (see, however, Sec. III C below for a further qualification of this question in terms of random perturbation probes).

\section{Pseudospectrum and operator resolvent}

To address the construction of pseudospectra, another characterization of the set $\sigma^{\epsilon}(A)$ in Eq. (31) of Definition 1 is very useful. Such a second characterization is based on the notion of the resolvent $R_{A}(\lambda)=(\lambda \mathrm{Id}-A)^{-1}$ of the operator $A$.

An eigenvalue $\lambda$ of $A$ is a complex number that makes the operator $(\lambda \mathrm{Id}-A)$ singular. More generally, the spectrum $\sigma(A)$ of $A$ is the set $\{\lambda \in \mathbb{C}\}$ for which the resolvent $R_{A}(\lambda)$ does not exist as a bounded operator (cf. details and subtleties on this notion in, e.g., Refs. [9,86]). This spectrum concept is a key notion for normal operators, but because of the spectral instabilities discussed above, $\sigma(A)$ is not necessarily a good object to consider for nonnormal operators, in our context. The notion of $\epsilon$ pseudospectrum then enters the scene. Specifically, an equivalent characterization of the $\epsilon$-pseudospectrum set $\sigma^{\epsilon}(A)$ in Definition 1 is given by the following definition [6,9].

Definition 2: (Pseudospectrum: resolvent norm approach). Given $A \in M_{n}(\mathbb{C})$, its resolvent $R_{A}(\lambda)=$ $(\lambda \mathrm{Id}-A)^{-1}$ and $\epsilon>0$, the $\epsilon$-pseudospectrum $\sigma^{\epsilon}(A)$ of $A$ is characterized as

$\sigma^{\epsilon}(A)=\left\{\lambda \in \mathbb{C}:\left\|R_{A}(\lambda)\right\|=\left\|(\lambda \mathrm{Id}-A)^{-1}\right\|>1 / \epsilon\right\}$.
This characterization captures the fact that, for nonnormal operators, the norm of the resolvent $R_{A}(\lambda)$ can be very large far from the spectrum $\sigma(A)$, which is in contrast with the normal-operator case, where (in the $\|\cdot\|_{2}$ norm)

$$
\left\|R_{A}(\lambda)\right\|_{2} \leq \frac{1}{\operatorname{dist}(\lambda, \sigma(A))} .
$$

In the non-normal case, one can only guarantee (e.g., Ref. [6]) that

$$
\left\|R_{A}(\lambda)\right\|_{2} \leq \frac{\kappa}{\operatorname{dist}(\lambda, \sigma(A))},
$$

where $\kappa$ is also a condition number, which is different from but related to the eigenvalue condition numbers $\kappa_{i}$ in Eq. (30) $(\kappa$, associated with the matrix diagonalizing $A$, provides an upper bound to the individual $\kappa_{i}$ 's; see Ref. [6] for details). In the non-normal case, $\kappa$ can become very large, and $\epsilon$-pseudospectra sets can extend far from the spectrum of $A$ for small values of $\epsilon$. The extension of $\sigma^{\epsilon}(A)$ far from $\sigma(A)$ is therefore a signature of strong nonnormality, and it indicates poor analytic behavior of $R_{A}(\lambda)$.

The important point here is that the characterization of the $\epsilon$-pseudospectrum in Definition 2, namely, Eq. (32), provides a practical way of calculating $\sigma^{\epsilon}(A)$. If we calculate the norm of the resolvent $\left\|R_{A}(\lambda)\right\|$ as a function of $\lambda=\operatorname{Re}(\lambda)+i \operatorname{Im}(\lambda) \in \mathbb{C}$, this provides a real function of two real variables $[\operatorname{Re}(\lambda), \operatorname{Im}(\lambda)]$ : The boundaries of the $\sigma^{\epsilon}(A)$ sets are just the "contour lines" of the plot of this function $\left\|R_{A}(\lambda)\right\|$. In particular, $\epsilon$-pseudospectra are nested sets in $\mathbb{C}$ around the spectrum $\sigma(A)$, with $\epsilon$ decreasing towards the "interior" of such sets, such that $\lim _{\epsilon \rightarrow 0} \sigma^{\epsilon}(A)=\sigma(A)$.

\section{Pseudospectrum and quasimodes}

For completeness, we provide a third equivalent characterization of the pseudospectrum in the spirit of characterizing $\lambda$ 's in the $\epsilon$-pseudospectrum set $\sigma^{\epsilon}(A)$ as "approximate eigenvalues" of $A$, "up to an error" $\epsilon$, with corresponding "approximate (right) eigenvectors" $v$. Specifically, it holds [6,9] that $\sigma^{\epsilon}(A)$ can also be characterized by the following (third) definition.

Definition 3 (Pseudospectrum: quasimode approach). Given $A \in M_{n}(\mathbb{C})$ and $\epsilon>0$, the $\epsilon$-pseudospectrum $\sigma^{\epsilon}(A)$ of $A$ and its associated $\epsilon$-quasimode $v \in \mathbb{C}^{n}$ are characterized by

$$
\sigma^{\epsilon}(A)=\left\{\lambda \in \mathbb{C}, \exists v \in \mathbb{C}^{n}:\|A v-\lambda v\|<\epsilon\right\} .
$$

This characterization introduces the notion of $\epsilon$-quasimode $v$ (referred to as a "pseudo-mode" in Ref. [6]), a key notion in the semiclassical analysis approach to the spectral study of $A$ [9]. On the other hand, this third characterization also clearly indicates the numerical difficulty that may occur when trying 
to determine the actual eigenvalues of $A$ since roundoff errors are unavoidable. This signals the need for a careful treatment when numerically addressing the spectral problem of a nonnormal operator $A$.

\section{Pseudospectrum and choice of norm}

In this subsection, we have presented the $\epsilon$ pseudospectrum as a notion that may be more adapted to the analysis of non-normal operators than that of the spectrum. We must emphasize, however, that the notion of spectrum $\sigma(A)$ is intrinsic to the operator $A$, whereas the $\epsilon$-pseudospectrum $\sigma^{\epsilon}(A)$ is not since it also depends on the choice of an operator norm. This fact is crucial since it determines what we mean by big or small when referring to the perturbation $\delta A$; therefore, it critically impacts the assessment of stability: A small operator perturbation $\delta A$ in a given norm can be a large one when considering another norm. In the first case, from a large variation $\delta \lambda$ in the eigenvalues, we can conclude instability, whereas, in the second case, such a variation could be consistent with stability.

In this sense, from a mathematical perspective, the study of spectral (in)stability through pseudospectra amounts to the identification of the proper scalar product determining the norm, that is, to the identification of the proper Hilbert space in which the operator $A$ acts. However, from a physical perspective, we might not have such freedom to choose the norm-conveniently rescaled on mathematical groundssince what we mean by large and small may be fixed by the physics of the problem, e.g., by the size of the involved amplitudes or intensities or the energy contained in the perturbations. Then, the choice of an appropriate norm, both from a mathematical and a physical perspective, is a fundamental step in the analysis (cf. discussion in Ref. [83]). This is the rationale behind the choice of the energy norm $\|\cdot\|_{E}$ in Eq. (20). Once the norm is chosen, the equivalent characterizations in Definitions 1-3, respectively, Eqs. (31), (32), and (35), emphasize the complementary aspects of the $\epsilon$-pseudospectrum notion and the $\sigma^{\epsilon}(A)$ sets.

\section{Pseudospectrum and random perturbations}

When considering the construction of pseudospectra, we have presented the characterization of $\sigma^{\epsilon}(A)$ in terms of the resolvent $R_{A}(\lambda)$ in Definition 2, Eq. (32), as better suited than the one in terms of spectra of perturbed operators in Definition 1, Eq. (31). The reason for this choice is that the former involves only the unperturbed operator $A$, whereas the latter demands a study of the spectral problem for any perturbed operator $A+\delta A$ with small $\delta A$ : A priori, the difficulty to explicitly control such space of possible $\delta A$ perturbations hinders an approach based on such characterization in Definition 1.

However, the very nature of the obstacle suggests a possible solution, namely, to consider the systematic study of the perturbed spectral problem under random perturbations $\delta A$ as an avenue to explore $\epsilon$-pseudospectra sets.
This heuristic expectation actually withstands a more careful analysis and constitutes the basis of a rigorous approach to the analysis of pseudospectra [9]. From a practical perspective, the systematic study of the spectral problem of $A+\delta A$ with (bounded) random $\delta A$, with $\|\delta A\| \leq \epsilon$, has proven to be an efficient tool to explore the "migration" of eigenvalues through the complex plane (inside the $\epsilon$-pseudospectra) [6]. This is complementary to (and technically independent from) the evaluation of $\sigma^{\epsilon}(A)$ from the contour lines of the norm $\left\|R_{A}(\lambda)\right\|$ of the resolvent. Such complementarity of approaches will be key later in our analysis of Nollert and Price's high-frequency perturbations of the Schwarzschild potential and the related QNMs.

Two important by-products of this random perturbation approach to the pseudospectrum are the following:

(i) Random perturbations help identify instabilitytriggering perturbations: $\epsilon$-pseudospectra and condition numbers $\kappa_{i}$ are efficient in identifying the instability of the spectrum and/or of a particular eigenvalue $\lambda_{i}$, respectively. However, they do not inform on the specific kind of perturbation actually triggering the instability, which can be crucial to assess the physical nature of the instability found. The use of families of random operators adapted to specific types of perturbations sheds light on this precise point. We will make critical use of this in our assessment of Schwarzschild's QNM (in)stability.

(ii) Random perturbations improve analyticity: A remarkable and apparently counterintuitive effect of random perturbations is the improvement of the analytic behavior of $R_{A}(\lambda)$ in $\lambda \in \mathbb{C}$ [9]. In particular, the norm $\left\|R_{A}(\lambda)\right\|$ is reduced away from $\sigma(A)$, as for normal operators [cf. Eq. (33)], so the $\epsilon$-pseudospectra sets pattern becomes "flattened" (a signature of good analytic behavior) below the random perturbation scale $\epsilon$.

To complement this perspective on the relation between the two given approaches to spectral (in)stability, namely, perturbation theory and $\epsilon$-pseudospectra (respectively, Secs. III A and III B), let us connect eigenvalue condition numbers $\kappa\left(\lambda_{i}\right)$ with $\epsilon$-pseudospectra $\sigma^{\epsilon}(A)$. The question we address is as follows: How far away can the $\epsilon$ pseudospectrum $\sigma^{\epsilon}(A)$ get from the spectrum $\sigma(A)$ ? The $\kappa_{i}^{\prime}$ 's provide the answer.

Let us define the "tubular neighborhood" $\Delta_{\epsilon}(A)$ of radius $\epsilon$ around the spectrum $\sigma(A)$ as

$$
\Delta_{\epsilon}(A)=\{\lambda \in \mathbb{C}: \operatorname{dist}(\lambda, \sigma(A))<\epsilon\},
$$

which is always contained in the $\epsilon$-pseudospectrum $\sigma^{\epsilon}(A)[6]$,

$$
\Delta_{\epsilon}(A) \subseteq \sigma^{\epsilon}(A) .
$$

The key question is about the inclusion in the other direction. Normal operators indeed satisfy [6] 


$$
\sigma_{2}^{\epsilon}(A)=\Delta_{\epsilon}(A)
$$

where $\sigma_{2}^{\epsilon}(A)$ indicates the use of a $\|\cdot\|_{2}$ norm. In other words, a $(\|\delta A\|<\epsilon)$ perturbed eigenvalue of a normal operator can move up to a distance $\epsilon$ from $\sigma(A)$. This is precisely what we mean by spectral stability, namely, that an operator perturbation of order $\epsilon$ induces an eigenvalue perturbation also of order $\epsilon$. However, in the non-normal case, where $\kappa\left(\lambda_{i}\right)>1$, it holds (for small $\epsilon$ ) [6] that

$$
\sigma^{\epsilon}(A) \subseteq \Delta_{\epsilon \kappa}(A):=\bigcup_{\lambda_{i} \in \sigma(A)} \Delta_{\epsilon \kappa\left(\lambda_{i}\right)+O\left(\epsilon^{2}\right)}\left(\left\{\lambda_{i}\right\}\right),
$$

so $\sigma^{\epsilon}(A)$ can extend into a much larger tubular neighborhood of radius about $\epsilon \kappa\left(\lambda_{i}\right)$ around each eigenvalue, signaling spectral instability if $\kappa\left(\lambda_{i}\right) \gg 1$. This bound is the essential content of the Bauer-Fike theorem relating pseudospectra and eigenvalue perturbations (cf. Ref. [6] for a precise formulation).

\section{NUMERICAL APPROACH: CHEBYSHEV'S SPECTRAL METHODS}

The present work is meant as a first assessment of $\mathrm{BH}$ QNM (in)stability by using pseudospectra. At this exploratory stage, we address the construction of pseudospectra in a numerical approach. As indicated in Sec. III B 3, the study of the spectral stability of non-normal operators is a challenging problem that demands high accuracy. Spectral methods provide well-adapted tools for these calculations $[6,89,90]$.

We discretize the differential operator $L$ in Eqs. (9)-(14) via Chebyshev differentiation matrices, built on Chebyshev-Lobatto $n$-point grids, producing $L^{N}$ matrix approximates (we note, systematically, $n=N+1$ in spectral grids, cf. Appendix C). Once the operator is discretized, the construction of the pseudospectrum requires the evaluation of matrix norms. A standard practical choice [6,89] involves the matrix norm induced from the Euclidean $L^{2}$ norm in the vector space $\mathbb{C}^{n}$ that, starting from Eq. (32) in Definition 2 of the pseudospectrum, leads to the following rewriting $[6,89]$ :

$$
\sigma_{2}^{\epsilon}(A)=\left\{\lambda \in \mathbb{C}: \sigma^{\min }(\lambda \mathrm{Id}-A)<\epsilon\right\},
$$

where $\sigma^{\min }(M)$ denotes the smallest singular value of $M$, that is, $\sigma^{\min }(M)=\min \left\{\sqrt{\lambda}: \lambda \in \sigma\left(M^{*} M\right)\right\}$, with $M \in$ $M_{n}(\mathbb{C})$ and $M^{*}$ its conjugate transpose $M^{*}=\bar{M}^{t}$.

Although Eq. (40) captures the spectral instability structure of $A$, the involved $L^{2}$ scalar product in $\mathbb{C}^{n}$ is neither faithful to the structure of the operator $L$ in Eq. (9) nor to the physics of the BH QNM problem (cf. discussion in Sec. III B 4). Instead, we use the natural norm in the problem, specifically, the Chebyshev-discretrized version of the "energy norm" (20), following from the Chebyshevdiscretized version of the scalar product (22). Specifically, we write the discretized scalar product in an appropriate basis as [we abuse the notation since we use $\langle\cdot, \cdot\rangle_{E}$ as in Eq. (22), although this is now a scalar product in a finitedimensional space $\mathbb{C}^{n}$ ]

$\langle u, v\rangle_{E}=\left(u^{*}\right)^{i} G_{i j}^{E} v^{j}=u^{*} \cdot G^{E} \cdot v, \quad u, v \in \mathbb{C}^{n}$,

where $G_{i j}^{E}$ is the Gram matrix corresponding to Eq. (22) (cf. Appendix C for its construction) and we note $u^{*}=\bar{u}^{t}$. The adjoint $A^{\dagger}$ of $A$ with respect to $\langle\cdot, \cdot\rangle_{E}$ is then written

$$
A^{\dagger}=\left(G^{E}\right)^{-1} \cdot A^{*} \cdot G^{E} .
$$

The vector norm $\|\cdot\|_{E}$ in $\mathbb{C}^{n}$ associated with $\langle\cdot, \cdot\rangle_{E}$ in Eq. (41) induces a matrix norm $\|\cdot\|_{E}$ in $M_{n}(\mathbb{C})$ [again, we abuse notation by using the same symbol for the norm in $\mathbb{C}^{n}$ and in $M_{n}(\mathbb{C})$ ]. Then (cf. Appendix B), the $\epsilon$ pseudospectrum $\sigma_{E}^{\epsilon}(A)$ of $A \in M_{n}(\mathbb{C})$ in the norm $\|\cdot\|_{E}$ is written

$$
\sigma_{E}^{\epsilon}(A)=\left\{\lambda \in \mathbb{C}: s_{E}^{\min }(\lambda \mathrm{Id}-A)<\epsilon\right\},
$$

where $s_{E}^{\min }$ is the smallest of the generalized singular values,

$$
s_{E}^{\min }(M)=\min \left\{\sqrt{\lambda}: \lambda \in \sigma\left(M^{\dagger} M\right)\right\},
$$

with $M \in M_{n}(\mathbb{C})$ and its adjoint $M^{\dagger}$ given by Eq. (42).

\section{TOY MODEL: PÖSCHL-TELLER POTENTIAL}

As presented in the previous sections, in our study of $\mathrm{BH}$ QNMs and their (in)stabilities, we exploit the geometrical framework of the hyperboloidal approach to analytically impose the physical boundary conditions at the BH horizon and at the radiation zone (future null infinity). As discussed in Sec. II, a crucial feature of such a strategy is that it allows us to cast the calculation of the QNM spectrum explicitly as the spectral problem of a non-self-adjoint differential operator, which is then the starting point for the tools used to assess spectral instabilities as presented in Sec. III, namely, the construction of the pseudospectrum and the analysis of random perturbations. Finally, spectral methods discussed in Sec. IV are employed to study these spectral issues through a discretization for the derivative operators. Prior to the study of the $\mathrm{BH}$ case, the goal of this section is to illustrate this strategy in a toy model, namely, the one given by the Pöschl-Teller potential.

\section{A. Hyperboloidal approach for the Pöschl-Teller potential}

The Pöschl-Teller potential [91], given by the expression

$\left.V(\bar{x})=\frac{V_{o}}{\cosh ^{2}(\bar{x})}=V_{o} \operatorname{sech}^{2}(\bar{x}), \quad \bar{x} \in\right]-\infty, \infty[$, 
has been widely used as a benchmark for the study of QNMs in the context of BH perturbation theory (e.g., Refs. [93-95]). Interestingly, QNMs of this potential have very recently been revisited, on the one hand, to illustrate the hyperboloidal approach to QNMs in a discussion much akin to the present one (cf. Ref. [81], cast in the setting of de Sitter spacetime) or, on the other hand, to illustrate functional analysis key issues related to the self-adjointness of the relevant operator [96]. Our interest in the PöschlTeller potential stems from the fact that it shares the fundamental behavior regarding QNM (in)stability to be encountered later in the $\mathrm{BH}$ context but in a mathematically much simpler setting. In particular, the Pöschl-Teller potential presents weaker singularities than the ReggeWheeler and Zerilli potentials in Schwarzschild spacetime, which translates to the absence of a continuous part of the spectrum of the relevant operator $L$ (corresponding to the "branch cut" in standard approaches to QNMs).

Let us consider the compactified hyperboloids given by Bizoń-Mach coordinates $[97,98]$ mapping $\mathbb{R}$ to $]-1,1[$,

$$
\left\{\begin{array}{l}
\tau=\bar{t}-\ln (\cosh \bar{x}) \\
x=\tanh \bar{x}
\end{array}\right.
$$

or, equivalently,

$$
\left\{\begin{array}{l}
\bar{t}=\tau-\frac{1}{2} \ln \left(1-x^{2}\right) \\
\bar{x}=\operatorname{arctanh}(x) .
\end{array}\right.
$$

In the spirit of the conformal compactification along the hyperboloids described in Sec. II B, we add the two points at (null) infinity (no BH horizon here), namely, $x= \pm 1$; thus, we work with the compact interval $[a, b]=[-1,1]$. Under this transformation, the wave equation (4) reads

$$
\left(\left(1-x^{2}\right)\left(\partial_{\tau}^{2}+2 x \partial_{\tau} \partial_{x}+\partial_{\tau}+2 x \partial_{x}-\left(1-x^{2}\right) \partial_{x}^{2}\right)+V\right) \phi=0,
$$

namely, the version of Eq. (7) corresponding to the transformation (46). We notice that angular labels $(\ell, m)$ are not relevant in the one-dimensional Pöschl-Teller problem. If $x \neq 1$, we can divide by $\left(1-x^{2}\right)$, and, defining

$$
\tilde{V}(x)=\frac{V}{\left(1-x^{2}\right)},
$$

we can write

$$
\left(\partial_{\tau}^{2}+2 x \partial_{\tau} \partial_{x}+\partial_{\tau}+2 x \partial_{x}-\left(1-x^{2}\right) \partial_{x}^{2}+\tilde{V}\right) \phi=0 .
$$

This expression is formally valid for any given potential $V(\bar{x})$ (although analyticity issues may appear if the asymptotic decay is not sufficiently fast, as is indeed the case for Schwarzschild potentials at $\mathcal{I}^{+}$). If we now insert the
Pöschl-Teller expression (45) and notice that $\operatorname{sech}^{2}(\bar{x})=$ $1-x^{2}$, we get a remarkably simple effective potential $\tilde{V}$, which is actually a constant:

$$
\tilde{V}(x)=V_{o} .
$$

In particular, the Pöschl-Teller wave equation (50) exactly corresponds to Eq. (4) in Ref. [81], so the Pöschl-Teller problem is equivalent to the Klein-Gordon equation in de Sitter spacetime with mass $m^{2}=V_{o}$. In the following, we choose $\lambda=1 / \sqrt{V_{o}}$ in the rescaling (5), so we can set

$$
\tilde{V}=1
$$

Now performing the first-order reduction in time [Eqs. (8) and (9)], for $w(x), p(x), q(x)$, and $\gamma(x)$ in Eq. (12), we get the values

$$
\begin{aligned}
& w(x)=1, \quad p(x)=\left(1-x^{2}\right), \\
& q(x)=\tilde{V}=1, \quad \gamma(x)=-x .
\end{aligned}
$$

Therefore, the operators $L_{1}$ and $L_{2}$ building the operator $L$ in Eq. (10) are written, in the Pöschl-Teller case, as

$$
\begin{aligned}
& L_{1}=\partial_{x}\left(\left(1-x^{2}\right) \partial_{x}\right)-1, \\
& L_{2}=-\left(2 x \partial_{x}+1\right) .
\end{aligned}
$$

As discussed in Sec. II C 1 , the function $p(x)=1-x^{2}$ vanishes at the boundaries of the interval $[a, b]=[-1,1]$, defining a singular Sturm-Liouville operator. This fact is at the basis of the absence of boundary conditions, if sufficient regularity is enforced on the eigenfunctions of the spectral problem. Therefore, regularity encodes the outgoing boundary conditions (see below). Finally, the scalar product (22) in this case is written as

$$
\begin{aligned}
\left\langle u_{1}, u_{2}\right\rangle_{E} & =\left\langle\left(\begin{array}{c}
\phi_{1} \\
\psi_{1}
\end{array}\right),\left(\begin{array}{c}
\phi_{2} \\
\psi_{2}
\end{array}\right)\right\rangle_{E} \\
& =\frac{1}{2} \int_{-1}^{1}\left(\bar{\psi}_{1} \psi_{2}+\left(1-x^{2}\right) \partial_{x} \bar{\phi}_{1} \partial_{x} \phi_{2}+\bar{\phi}_{1} \phi_{2}\right) d x .
\end{aligned}
$$

\section{B. Pöschl-Teller QNM spectrum}

\section{Exact Pöschl-Teller QNM spectrum}

The Pöschl-Teller QNM spectrum can be obtained by solving the eigenvalue problem in Eqs. (14) and (15), with operators $L_{1}$ and $L_{2}$ given by Eq. (54). As commented above, no boundary conditions need to be added if we enforce the appropriate regularity. In this particular case, the eigenvalue problem can be solved exactly. The resolution itself is informative since it illustrates this regularity issue concerning boundary conditions. 
If we substitute the first component of Eq. (15) into the second or, simply, if we take the Fourier transform in $\tau$ in Eq. (50) [with $\tilde{V}=1$ from the chosen $\lambda$ leading to Eq. (52)], we get

$$
\left[\left(1-x^{2}\right) \frac{d^{2}}{d x^{2}}-2(i \omega+1) x \frac{d}{d x}-i \omega(i \omega+1)-1\right] \phi=0 .
$$

This equation can be solved in terms of the hypergeometric function ${ }_{2} F_{1}(a, b ; c ; z)$, with $z=(1-x / 2)$ (see details in Appendix D). In particular, for each value of the spectral parameter $\omega$, we have a solution that can be written as a linear combination of linearly independent solutions obtained from ${ }_{2} F_{1}(a, b ; c ; z)$. Discrete QNMs are obtained only when we enforce the appropriate regularity that encodes the outgoing boundary conditions. In this case, it is obtained by enforcing the solution to be analytic in $x \in[-1,1]$ (corresponding, in $z$, to analyticity in the full closed interval $[0,1])$, which amounts to truncating the hypergeometric series to a polynomial. We emphasize that such a need for truncating the infinite series to a polynomial, a familiar requirement encountered in many different physical settings, embodies the enforcement of outgoing boundary conditions. In summary, this strategy leads to the Pöschl-Teller QNM frequencies (cf. e.g., Refs. $[92,94])$

$$
\omega_{n}^{ \pm}= \pm \frac{\sqrt{3}}{2}+i\left(n+\frac{1}{2}\right)
$$

with corresponding QNM eigenfunctions in this setting,

$$
\phi_{n}^{ \pm}(x)=P_{n}^{\left(i \omega_{n}^{ \pm}, i \omega_{n}^{ \pm}\right)}(x), \quad x \in[-1,1],
$$

where $P_{n}^{(\alpha, \beta)}$ are the Jacobi polynomials (see Appendix D). Two comments are in order here:

(i) QNMs are normalizable: QNM eigenfunctions $\phi_{n}^{ \pm}(x)$ are finite and regular when making $\bar{x} \rightarrow \pm \infty$, corresponding to $x= \pm 1$. This case is in contrast with the exponential divergence of QNM eigenfunctions in Cauchy approaches, where the time slices reach spatial infinity $i^{0}$. The regularity of $\phi_{n}^{ \pm}(x)$ is a direct consequence of the hyperboloidal approach with slices reaching $\mathcal{I}^{+}$. The resulting normalizability of the QNM eigenfunctions can be relevant in, e.g., resonant expansions (cf. e.g., discussion in Ref. [62]).

(ii) QNM regularity and outgoing conditions: In the present case, namely, the Pöschl-Teller potential in Bizon-Mach coordinates, analyticity (actually, the polynomial structure) implements the regularity enforcing outgoing boundary conditions. Analyticity is too strong in the general case. However, asking for smoothness is not enough (see, e.g., Ref. [75]). In Refs. [78-80], this problem is approached in terms of Gevrey classes, which interpolate between analytic and (smooth) $C^{\infty}$ functions, identifying the space of $(\sigma, 2)$-Gevrey functions as the proper regularity notion. The elucidation of the general adequate functional space for QNMs, tantamount to the consistent implementation of outgoing boundary conditions, is crucial for the characterization of QNMs in the hyperboloidal approach.

\section{Numerical Pöschl-Teller QNM spectrum}

Figure 3 shows the result of the numerical counterpart of the Pöschl-Teller eigenvalue calculation, whose exact discussion has been presented above, by using the discretized operators $L, L_{1}$, and $L_{2}$ described in Sec. IV and Appendix C,

$$
L^{N} v_{n}^{(N)}=\omega_{n}^{(N)} v_{n}^{(N)}
$$

Indeed, this approach numerically recovers the analytical result in Eq. (57) (we drop the " \pm " label, focusing on one of the branches that are symmetric with respect to the vertical axis).

We stress that the remarkable agreement between the numerical values from the bottom panel of Fig. 3 (see also Fig. 7 later) and the exact expression (57) is far from being a trivial result, as already illustrated in existing systematic numerical studies. In particular, this is the case of Ref. [99] (where the Pöschl-Teller potential is referred to as the
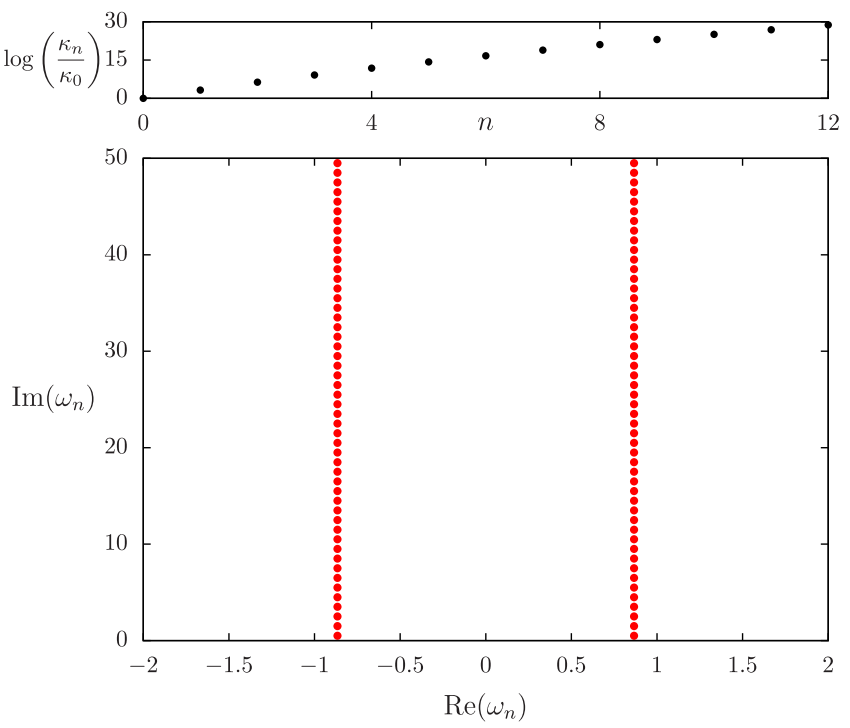

FIG. 3. Pöschl-Teller QNM problem. Bottom panel: QNM spectrum for the Pöschl-Teller potential, calculated in the hyperboloidal approach described in Sec. VA, with Chebyshev spectral methods and enhanced machine precision. Top panel: ratios of condition numbers $\kappa_{n}$ of the first QNMs over the condition number $\kappa_{0}$ of the fundamental QNM, indicating a growing spectral instability compatible with the need for using enhanced machine precision. 
Eckart barrier potential), where the fundamental mode $\omega_{0}^{ \pm}$ in Eq. (57) is stable and accurately recovered, whereas all overtones $\omega_{n \geq 1}^{ \pm}$suffer from a strong instability (triggered, according to the discussion in Refs. $[99,100]$, by the $C^{1}$ regularity of the approximation modeling the Pöschl-Teller potential) and cannot be recovered.

In our setting, a convergence study of the numerical values shows that the relative error

$$
\mathcal{E}_{n}^{(N)}=\left|1-\frac{\omega_{n}^{(N)}}{\omega_{n}}\right|
$$

between the exact QNM $\omega_{n}$ and the corresponding numerical approximation $\omega_{n}^{(N)}$ (obtained at a given truncation $N$ of the differential operator) actually increases with the resolution. This increase is a first hint of the instabilities to be discussed later. Indeed, the top panel of Fig. 4 displays the error for the fundamental mode $n=0$ and the first
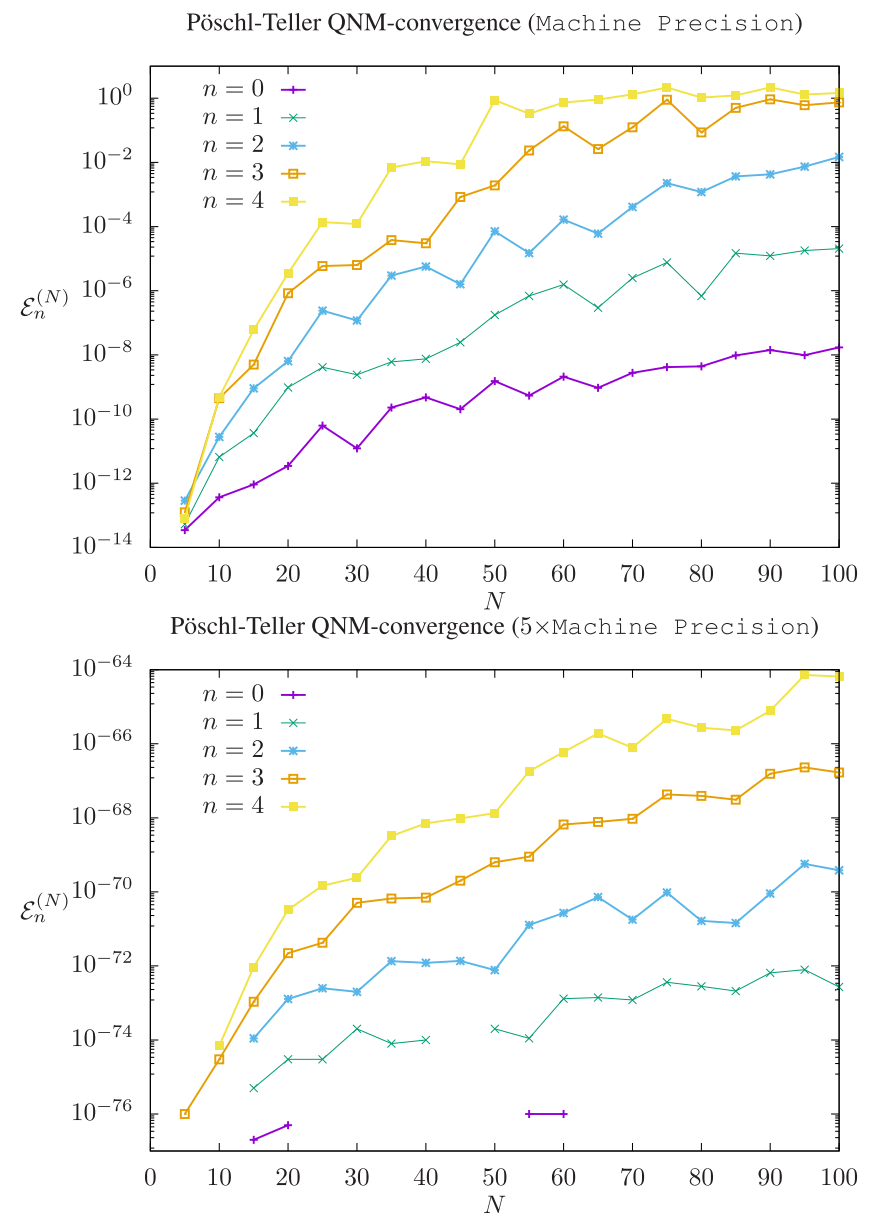

FIG. 4. Convergence test for the Pöschl-Teller QNM. Top panel: double floating point operations with internal roundoff error set to machine precision. Bottom panel: double floating point operations with internal roundoff error set to $5 \times$ machine precision. Note that missing points for $n=0$ correspond to errors that exactly vanish at the employed machine precision. overtones $n=1, \ldots, 4$ when the eigenvalue problem for the discretized operator is naively solved with the standard machine roundoff error for floating point operations (typically, about $10^{-16}$ for double precision).

It is astonishing how, despite the simplicity of the exact solution, the relative error grows significantly already for the first overtones and, crucially, more strongly as the damping grows with higher overtones. To mitigate such a drawback, one needs to modify the numerical treatment in order to allow for a smaller roundoff error in floating point operations. The bottom panel of Fig. 4 shows the error $\mathcal{E}_{n}^{(N)}$ when the calculations are performed with an internal roundoff error according to $5 \times$ machine precision, i.e., about $10^{-5 \times 16}$. In this case, the fundamental QNM $n=0$ is "exactly" calculated at the numerical level (i.e., the difference between its exact value and the numerical approximation vanishes at this precision). The error for the overtones still grows but in a safe range, for all practical purposes. The values displayed in the bottom panel of Fig. 3 were obtained with an internal roundoff error set to $10 \times$ machine precision, and we can assure that the errors of all overtones are smaller than $10^{-100}$.

\section{Condition numbers of QNM frequencies}

The growth in the relative error as we move to higher overtones in Fig. 4 suggests an increasing spectral instability in $n$ of eigenvalues $\omega_{n}^{ \pm}$, triggered by numerical errors related to machine precision. Thus, this instability can be reduced (but not eliminated) by improving the internal roundoff error.

At the level of the nonperturbed spectral problem (59), and in order to assess, more systematically, such spectral instability, we can apply the discussion in Sec. III A to the Pöschl-Teller approximates $L^{N}$. Namely, solving the righteigenvector problem (59), together with the left-eigenvector one,

$$
\left(L^{N}\right)^{\dagger} w_{n}^{(N)}=\bar{\omega}_{n}^{(N)} w_{n}^{(N)},
$$

we can compute the condition numbers $\kappa_{n}^{(N)}=\kappa\left(\omega_{n}^{(N)}\right)=$ $\left\|v_{n}^{(N)}\right\|_{E}\left\|w_{n}^{(N)}\right\|_{E} /\left|\left\langle v_{n}^{(N)}, w_{n}^{(N)}\right\rangle_{E}\right|$ introduced in Eq. (30). Notice that this calculation is quite nontrivial since it involves, first, the construction of the adjoint operator $\left(L^{N}\right)^{\dagger}=\left(G^{E}\right)^{-1} \cdot\left(L^{N}\right)^{*} \cdot G^{E}$ and, second, the calculation of scalar products $\langle\cdot, \cdot\rangle_{E}$ and (vector) energy norms $\|\cdot\|_{E}$. These calculations involve the determination of the Gram matrix $G^{E}$ associated with the energy scalar product (55) by implementing expression (C23) in Appendix C. These expressions are quite nontrivial, and in the following section, we provide a strong test for the associated analytical and numerical construction.

The result is shown in the top panel of Fig. 3. The ratio of the condition numbers $\kappa_{n}$, relative to the condition number of the fundamental mode $\kappa_{0}$, grows strongly with $n$, which 
indicates a strong and increasing spectral instability consistent with the error convergence displayed in Fig. 4. The rest of this section is devoted to addressing this spectral stability issue.

\section{Pöschl-Teller pseudospectrum}

\section{Motivating the pseudospectrum}

As the previous discussion makes apparent, a crucial question that arises after obtaining the QNM spectrum of the operator $L$ in Eq. (10), with $L_{1}$ and $L_{2}$ in Eq. (54), is whether such QNM eigenvalues are stable under small perturbations of $L$-more specifically for QNM physics, and in the context of the wave equation (4), whether the QNM spectrum is stable under small perturbations of the potential $V$. The latter is the specific type of perturbation we are assessing in this work.

In the numerical approach we have adopted, perturbations in the spectrum under small perturbations in $L$ may arise either from numerical noise resulting from the chosen discretization strategy, or they can originate from "realworld sources," namely, small physical perturbations of the considered potential $V$. Ultimately, in the BH setting for which the Pöschl-Teller potential provides a toy model, such physical perturbations could stem from a "dirty" environment surrounding a black hole and/or emergent fluctuations from quantum-gravity effects. Therefore, the question of whether QNM spectrum instability is a structural feature of the operator $L$-i.e., not just an artifact of a given numerical algorithm-is paramount for our understanding of the fundamental physics underlying the problem.

A pragmatic approach to address this question consists in explicitly introducing families of perturbations [101] and studying their effect on the QNM spectra themselves $[23,46-50,52]$. We discuss this approach later in Sec. V D, but first, we apply the pseudospectrum approach described in Sec. III B to the Pöschl-Teller problem. Indeed, one of the main goals of our present work is to bring attention to and emphasise the fact that the unperturbed operator already contains crucial information to assess such (in) stability features. We have already encountered this fact in the evaluation of the condition numbers $\kappa_{n}$ in Fig. 3, which only depends on the unperturbed operator $L$, but we develop this theme further with the help of the pseudospectrum notion. Indeed, pseudospectrum analysis provides a framework to identify the (potential) spectral instability, which is oblivious to the particular perturbation employed. Then, in a second stage, actual perturbations of the operators, with a particular emphasis on random perturbations along the lines in Sec. III C, can be used to complement and refine such pseudospectrum analysis.

Figure 5 shows the pseudospectrum for the Pöschl-Teller potential in the energy norm of Eq. (20) associated with the scalar product (55). Let us explain the content of such a figure. According to the characterization in Definition 1,

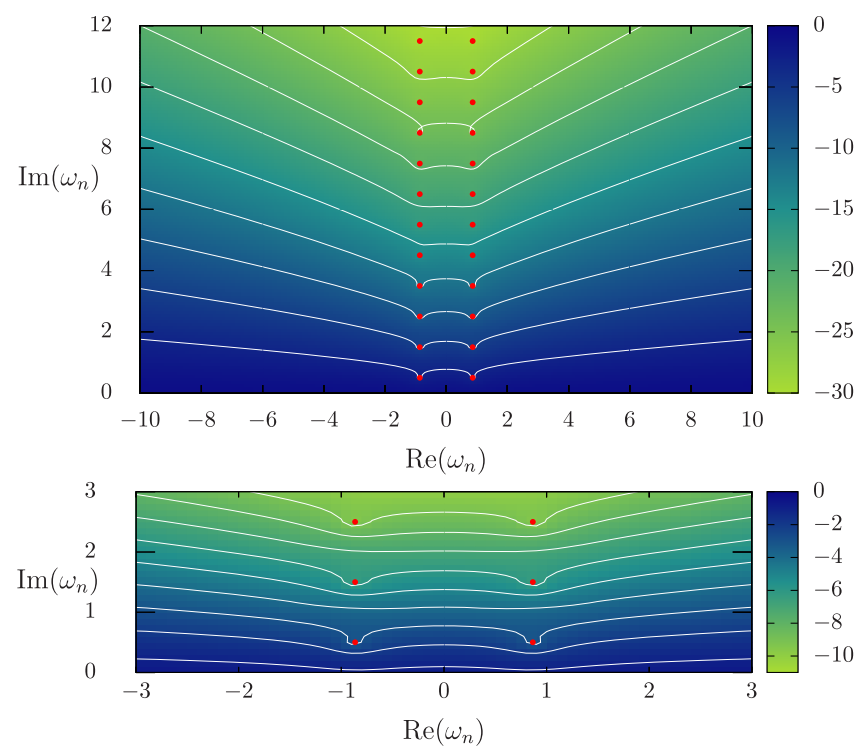

FIG. 5. Top panel: pseudospectrum for the Pöschl-Teller potential. QNMs (red circles) from Fig. 3 are superimposed, for reference, on their (in)stability. The color log scale corresponds to $\log _{10} \epsilon$, with white lines indicating the boundaries of $\epsilon$-pseudospectra sets $\sigma^{\epsilon}$, whose interior extends upwards in the $\omega$ complex plane. Bottom panel: zoom into the region around the fundamental QNM and first overtones.

namely, Eq. (31), of the $\epsilon$-pseudospectrum of the operator $L$, the set $\sigma^{\epsilon}(L)$ is the collection of all complex numbers $\omega \in \mathbb{C}$ that are actual eigenvalues for some operator $L+\delta L$, where $\delta L$ is a small perturbation of "size" smaller than a given $\epsilon>0$. Consequently and crucially, adding a perturbation $\delta L$ with $\|\delta L\|_{E}<\epsilon$ entails an actual ("physical") change in the eigenvalues $\omega_{n}$ that can reach up to the boundary of the $\sigma^{\epsilon}(L)$ set, marked by white lines in Fig. 5. The key question is to assess if $\epsilon$-pseudospectra for small $\epsilon$ can extend to large areas of $\mathbb{C}$ or not, which is tightly related to condition numbers $\kappa_{n}$ controlling eigenvalue spectral instabilities, as explicitly estimated by the Bauer-Fike relation (39) between $\epsilon$-pseudospectra sets and "tubular neighborhoods" $\Delta_{\kappa \epsilon}$ of radii $\epsilon \kappa_{n}$ around the spectrum. Let us first discuss a self-adjoint test case and, in a second stage, the actual non-self-adjoint case [105].

\section{Pseudospectrum: Self-adjoint case}

As discussed in Sec. II D, setting $L_{2}=0$ in Eq. (10)while keeping $L_{1}$ as in Eq. (54) - leads to a self-adjoint operator $L$ [106]. Therefore, the associated spectral problem is stable, cf. Sec. III A. A typical pseudospectrum in the self-adjoint (more generally, "normal") case is illustrated in Fig. 6: a "flat" pseudospectrum with large values of $\epsilon$ for $\epsilon$ pseudospectra sets, when moving "slightly" (in the $\mathbb{C}$ plane) away from the eigenvalues. Note also, in this case, the horizontal contour lines far from the spectrum, indicating that all eigenvalues share the same stability properties in the energy norm. 

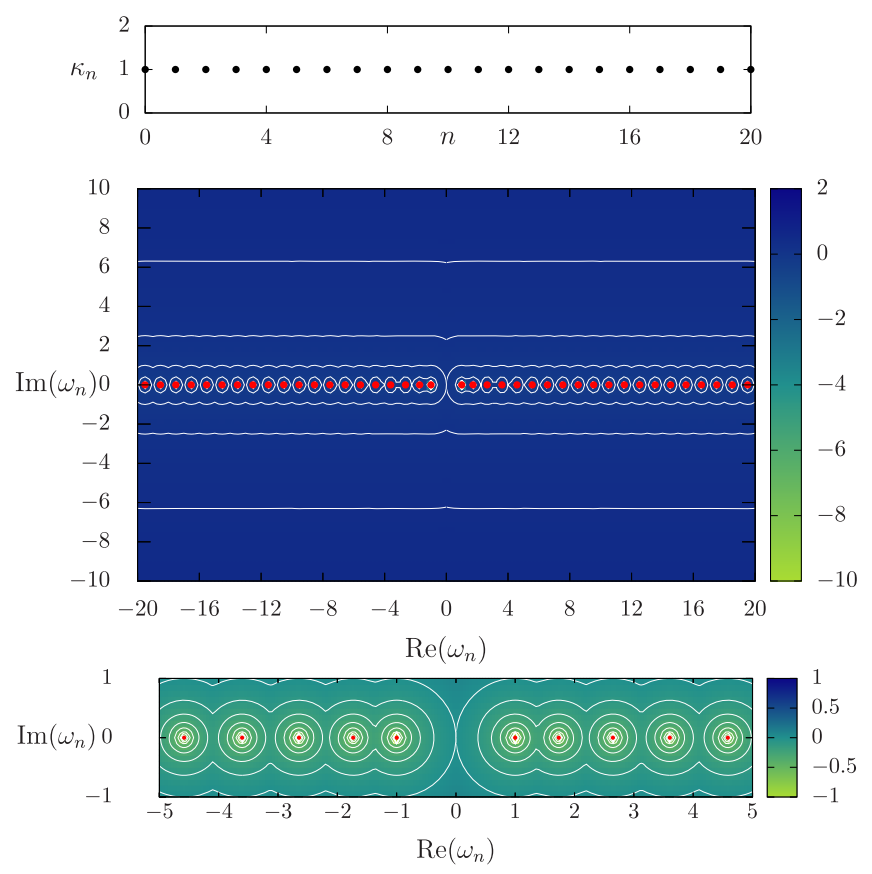

FIG. 6. Pseudospectrum and eigenvalue condition numbers of a self-adjoint operator (Pöschl-Teller potential with $L_{2}=0$ ). Top panel: condition numbers, $\kappa_{n}=1$ for $\omega_{n}(0 \leq n \leq 20)$. Middle panel: pseudospectrum, "flat" pattern typical of a spectrally stable (normal) operator. Bottom panel: zoom near the spectrum, with concentric circles ("radius $\epsilon$ " tubular regions around eigenvalues) characteristic of stability.

Let us describe Fig. 6 in more detail. Boundaries of $\epsilon$ pseudospectra $\sigma^{\epsilon}(A)$ are marked by white lines, with the $\epsilon$ 's corresponding to the values in the color log scale. Pseudospectra $\sigma^{\epsilon}(L)$ are, by construction, "nested sets" around the spectrum (red points in Fig. 6), the latter corresponding to the "innermost set" $\sigma^{\epsilon}(L)$ when $\epsilon \rightarrow 0$. In this self-adjoint case, condition numbers in Eq. (30) must satisfy $\kappa_{n}=1$, as we have verified and explicitly shown in the top panel of Fig. 6. Then, and consistently with Eq. (38), the corresponding nested sets $\sigma^{\epsilon}(L)$ are actually tubular regions $\Delta_{\epsilon}(L)$ of radius $\epsilon$ around the spectrum, so a change $\delta L$ with a norm of order $\epsilon$ in the operator $L$ entails a maximum change in the eigenvalues of the same order $\epsilon$. Specifically, $\epsilon$-pseudospectra sets show concentric circles around the spectra that quickly reach large-epsilon values, i.e., $\epsilon \sim O(1)$, when moving away from eigenvalues. As a consequence, one would need perturbations in the operator of the same order to dislodge the eigenvalues slightly away from their original values: Then, we say that $L$ is spectrally stable. Pseudospectra sets with small $\epsilon$ are then "tightly packed" in "thin throats" around the spectrum; thus, light green colors are indeed so close to spectrum "red points" that they are not visible in the scale of Fig. 6, giving rise to a typical "flat" pseudospectrum figure of a "single color."

Horizontal boundaries of $\epsilon$-pseudospectra, when far from the spectrum, are a consequence (in this particular problem) of using the energy norm. If another norm is used, e.g., the standard one induced from the $L^{2}$ norm in $\mathbb{C}^{n}$, the global "flatness" of the pseudospectrum is still recovered, especially when comparing with the corresponding scales in Fig. 5, indicating a much more stable situation than the general $L_{2} \neq 0$ case. But when refining the scale, one would observe that pseudospectra contour lines far from the spectrum are not horizontal but present a slope, growing with the frequency. This behavior indicates that, under perturbations of the same size in that $L^{2}$ norm, higher frequencies can move further than low frequencies, which is in tension with the equal stability of all the eigenvalues. What is going on is the effect commented on in Sec. III B 4 concerning the impact of the norm choice on the notions of big or small: When using the $L^{2}$ norm, we would mark, with the same small $\epsilon$, different perturbations among which there exist $\delta L$ instances that actually strongly excite the high frequencies, but such a feature is blind to the $L^{2}$ norm. However, if using a norm sensitive to high-frequency effects, as is the case of the energy norm that has a $H^{1}$ character incorporating derivative terms, those same perturbations $\delta L$ would have a norm much larger than $\epsilon$, with the derivative terms in the energy norm indeed weighing more as the frequency grows. A small perturbation $\delta L$ in the norm $L^{2}$ turns out to be a big one in the energy norm, so stronger modifications in the eigenvalues are indeed consistent with stability. In practice, in order to construct a given $\epsilon$-pseudospectrum set, such high-energy perturbations $\delta L$ need to be renormalized to keep $\epsilon$ fixed, something that the energy norm does automatically. This example shows how the choice of the norm affects the assessment of spectral stability, and, in particular, it shows the importance of the energy norm in the present work, namely, for high-frequency issues.

Figure 6 may appear uninteresting, but it is actually a tight and constraining test of our construction, both at the analytical and at the numerical level. First, the panels in Fig. 6 correspond to different calculations: The top panel results from an eigenvalue calculation (actually two, one for $L$ and another for $L^{\dagger}$ ), whereas the "map" in the middle and bottom panels is the result of calculating the energy norm of the resolvent $R_{L}(\omega)=(\omega \mathrm{Id}-L)^{-1}$ at each point $\omega \in \mathbb{C}$. Both calculations depend on the construction of the Gram matrix $G^{E}$ but are indeed different implementations. The $\kappa_{n}=1$ values in the top panel constitute a most stringent test since modifications in either the analytical structure of the scalar product (55) or the slightest mistake in the discrete counterpart (C23) spoil the result. As discussed at the end of Sec. V B 3, this provides a strong test of both the analytical treatment and the numerical discretization of the differential operator and scalar product. On the other hand, the plain flatness of the pseudospectrum in the middle panel is a strong test of the self-adjoint character of $L$ when $L_{2}=0$, which, given the subtleties of the spectral discretization explained in Appendix $\mathrm{C}$, provides a reassuring nontrivial test for the whole numerical scheme. 


\section{Non-self-adjoint case: Pöschl-Teller pseudospectrum}

In contrast with the self-adjoint case, when considering the actual $L_{2} \neq 0$ of the Pöschl-Teller case, pseudospectra sets $\sigma^{\epsilon}(L)$ with small $\epsilon$ extend in Fig. 5 into large regions of $\mathbb{C}$ (with typical sizes much larger than $\epsilon$ ); therefore, the operator $L$ is spectrally unstable: Very small (physical) perturbations $\delta L$, with $\|\delta L\|_{E}<\epsilon$, can produce large variations in the eigenvalues up to the boundary of the now largely extended region $\sigma^{\epsilon}(L)$. Such strong variations of the spectrum are not a numerical artifact, related, e.g., to machine precision; rather, they correspond to an actual structural property of the nonperturbed operator. Indeed, large values of the condition numbers $\kappa_{n}$ in the top panel of Fig. 3 show that the tubular sets $\Delta_{\epsilon \kappa}(L)$ in Eq. (36) now extend into large areas in $\mathbb{C}$. This fact about the $\kappa_{n}$ 's is consistent with the large regions in Fig. 5 corresponding to $\sigma^{\epsilon}(L)$ sets with very small $\epsilon$ 's. Such a nontrivial pattern of $\epsilon$-pseudospectra is a strong indication of spectral instability, although without a neat identification of the actual nature of the perturbations triggering instabilities.

\section{Reading pseudospectra: "Topographic maps" of the resolvent}

In practice, if one wants to read from pseudospectrasuch as those in Figs. 5 or 6 - the possible effect of a physical perturbation of (energy) norm of order $\epsilon$ on QNMs, one must first determine the white line corresponding to that $\epsilon$ (using the $\log$ scale). Then, eigenvalues can potentially move in the whole region bounded by that line (namely, the $\epsilon$-pseudospectrum set for the nonperturbed operator $L$ ), which, in Fig. 5, corresponds to the region above the white line.

Pseudospectra can actually be seen as a map of the analytical structure of the resolvent $R_{L}(\omega)=(\omega \mathrm{Id}-L)^{-1}$ of the operator $L$, taken as a function of $\omega$. This interpretation corresponds to the characterization in Definition 2 of the pseudospectrum, Eq. (32), which is indeed the one used to effectively construct the pseudospectrum [specifically, its realization (43) in the energy norm; cf. Appendix B 3 for details). From this point of view, the boundaries of the $\epsilon$-pseudospectra (white lines in Figs. 5 and 6) can be seen as contour lines of the height function $\left\|R_{L}(\omega)\right\|_{E}$, namely, the norm of the resolvent $R_{L}(\omega)$. In quite a literal sense, the pseudospectrum can then be read as a topographic map, with stability characterized by very steep throats around eigenvalues quickly reaching flat zones away from the spectrum, whereas instability corresponds to nontrivial topographic patterns extending into large regions of the map far away from the eigenvalues.

In summary, this topographic perspective makes it apparent that there is a stark contrast between the flat pattern of the self-adjoint case of Fig. 6, corresponding to stability, and the nontrivial pattern of the (non-self-adjoint) Pöschl-Teller pseudospectrum in Fig. 5-in particular, indicating a (strong) QNM sensitivity to perturbations that increases as damping grows.

\section{Pöschl-Teller perturbed QNM spectra}

Pseudospectra inform about the spectral stability and instability of an operator, but they do not identify the specific type of perturbation triggering instabilities. Therefore, in a second stage, it is illuminating to complement the pseudospectrum information with the exploration of spectral instability with perturbative probes into the operator, always under the perspective acquired with the pseudospectrum. A link between both pseudospectra and perturbation strategies is provided by the Bauer-Fike theorem [6], as expressed in Eq. (39).

\section{Physical instabilities: Perturbations in the potential $V$}

Not all possible perturbations of the $L$ operator are physically meaningful. One example, in the setting of our numerical approach, are the machine precision error perturbations $\delta L^{N}$ to the $L^{N}$ matrix. As discussed in Sec. V B 2, machine precision errors indeed trigger large deviations in the spectrum, consistently with the nontrivial pattern of the pseudospectrum in Fig. 5, but clearly, we should not consider such effects to be physical. They are a genuine numerical artifact since the structure of the perturbation $\delta L^{N}$ does not correspond to any physical or geometrical element in the problem.

The methodology we follow to address this issue is as follows: (i) Given a grid resolution $N$, we first set the machine precision to a value sufficiently high so as to guarantee that all nonperturbed eigenvalues are correctly recovered; (ii) we then add a prescribed perturbation with the specific structure corresponding to the physical aspect we are studying.

In the present work, we focus on a particular kind of perturbation, namely, perturbations to the potential $V$ and, more specifically, perturbations $\delta \tilde{V}$ to the rescaled potential $\tilde{V}$ in Eq. (21). This approach is similar to the study in Ref. [44]. In other words, we consider perturbations $\delta L$ to the $L$ operator of the form

$$
\delta L=\frac{1}{i}\left(\begin{array}{c|c}
0 & 0 \\
\hline \delta \tilde{V} / w(x) & 0
\end{array}\right)
$$

We note that, at the matrix level, the $\delta \tilde{V} / w(x)$ submatrix is just a diagonal matrix. Therefore, the structure of $\delta L$ in Eq. (62) is a very particular one. The pseudospectrum in Fig. 5 tells us that $L$ is spectrally unstable, and we know that machine precision perturbations trigger such instabilities. However, nothing guarantees that $L$ is actually unstable under a perturbation of the particular form in Eq. (62). It is a remarkable fact, crucial for our physical 
discussion, that $L$ is indeed unstable under such perturbations and, therefore, under perturbations of the potential $V$.

\section{Random and high-frequency perturbations in the potential $V$}

We have considered two types of generic, but representative, perturbations $\delta L$ of the form given in Eq. (62):

(i) Random perturbations $\delta \tilde{V}_{\mathrm{r}}$ : We set the perturbation according to a normal Gaussian distribution on the collocation points of the grid. By construction, this is a high-frequency perturbation. Random perturbations are a standard tool [6] to explore generic properties of spectral instability, and there indeed exists a rich interplay between pseudospectra and random perturbations [9].

(ii) Deterministic perturbations $\delta \tilde{V}_{\mathrm{d}}$ : We have chosen

$$
\delta \tilde{V}_{\mathrm{d}} \sim \cos (2 \pi k x),
$$

in order to address the specific impact of high- and lowfrequency perturbations in QNM spectral stability, by exploring the effect of changing the wave number $k$. Perturbations $\delta \tilde{V}$ are then rescaled so as to guarantee $\|\delta L\|_{E}=$ $\epsilon$ (abusing notation, in the following we write $\|\delta \tilde{V}\|_{E}$ instead of $\|\delta L\|_{E}$, in order to emphasize that the operator perturbation is restricted to the potential). The impact on QNM frequencies resulting from adding these perturbations is shown in Fig. 7. In both random and deterministic cases, the sequence ofimages in Fig. 7 shows a high-frequency instability of QNM overtones that "migrate" towards new QNM branches. The fundamental (slowest-decaying) QNM is, however, stable under these perturbations. More generally, such QNM instability is sensitive with respect to both the perturbation size and frequency.

Before further discussing the details of the QNM instability, namely, the nature of the new QNM branches, an important point must be addressed: whether the values obtained correspond to the actual eigenvalues of the new, perturbed operator $L+\delta L$, or whether they are an artifact of some numerical noise. As in the nonperturbed case discussed in Sec. V B 2, and as explained above when introducing the employed methodology, results are obtained with a high internal accuracy $(10 \times$ machine precision), so any numerical noise is below the range of values shown. Proceeding systematically, Fig. 8 presents the convergence tests for a few eigenvalues resulting from the deterministic perturbation (random perturbations do not admit this kind of test) with norm $\left\|\delta \tilde{V}_{\mathrm{d}}\right\|_{E}=10^{-8}$ and frequency $k=20$ (bottom-right panel of Fig. 7). The relative error is calculated as

$$
\mathcal{E}_{n}^{(N)}=\left|1-\frac{\omega_{n}^{(N)}}{\omega_{n}^{(N=400)}}\right| ;
$$

i.e., in the absence of exact results, we take, as reference, the values with a high resolution $N=400$. As representative QNMs, we have chosen the following: (a) The last "unperturbed" overtone, whose value is actually very close to the (truly) unperturbed QNM $\omega_{4}$.

(b) The first new QNM on the imaginary axis.

(c) Three QNMs along the new branch with values spread in the ranges $1 \lesssim \operatorname{Re}\left(\omega_{n}\right) \lesssim 10$ and $5 \lesssim \operatorname{Im}\left(\omega_{n}\right) \lesssim 8$.

One observes a systematic convergence, with the relative error dropping circa 10 orders of magnitude when the numerical resolution increases [107] from $N=150$ to $N=400$. This result confirms that the spectrum indeed corresponds to the new, perturbed operator and is not a numerical artifact, which neatly shows the unstable nature of the QNM spectrum of the unperturbed Pöschl-Teller operator: Eigenvalues indeed migrate to new branches under very small perturbations.

\section{Perturbed QNM branches and pseudospectrum}

High-frequency perturbations trigger the migration of QNM overtone frequencies to new perturbed QNM branches. Figure 9 displays the perturbed QNM spectra on top of the pseudospectra for the unperturbed operator. The remarkable predictive power of the pseudospectrum becomes apparent: Perturbed QNMs follow the boundaries of pseudospectrum sets. In other words, QNM overtones migrate to new branches closely tracking the $\epsilon$-pseudospectra contour lines. This happens for both random and deterministic high-frequency perturbations. Crucially, no such instability is observed for low-frequency deterministic perturbations, with small wave number $k$. Consequently, in the following, we refer to this effect as an ultraviolet instability of QNM overtones.

Remarkably, such high-frequency QNM instability is not limited to highly damped QNMs but indeed reaches the lowest overtones, with the random perturbations being more effective in reaching the slowest-decaying overtones for a given norm $\|\delta \tilde{V}\|_{E}=\epsilon$. This result is qualitatively consistent with the analyses in Refs. [45,52] for Dirac-delta potentials (compare, e.g., perturbed QNM branches in Fig. 9 here with Fig. 1 in Ref. [52]). These findings advocate the use of pseudospectra to probe QNM instability, demonstrating its capability to capture it already at the level of the nonperturbed operator. At the same time, pseudospectra are oblivious to the nature of the perturbation triggering instabilities. A complementary perturbation analysis, in particular, through random perturbations, is then necessary to identify the high-frequency nature of the instability, confirming its physicality in the sense of being associated with actual perturbations of the potential $V$.

\section{High-frequency stability of the slowest-decaying QNM}

The high-frequency instability observed for QNM overtones is absent in the fundamental QNM. The slowestdecaying QNM is therefore ultraviolet stable. Such a stability is already apparent in the pseudospectrum in Fig. 5, where the order of the $\epsilon$ 's corresponding to $\epsilon$-pseudospectra sets around the fundamental QNM reaches 

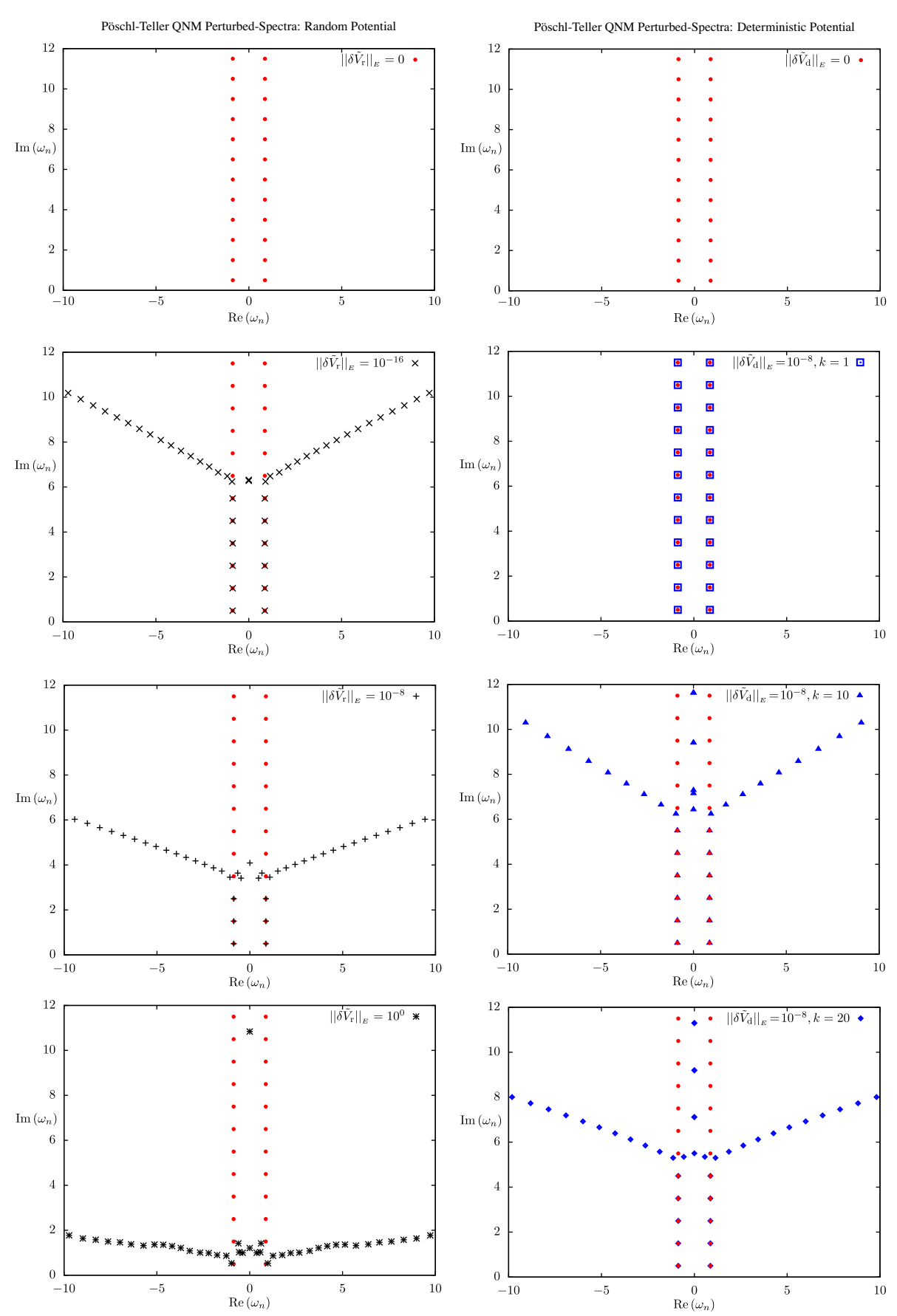

FIG. 7. Left column: sequence of QNM spectra for the Pöschl-Teller potential subject to a random perturbation $\delta \tilde{V}_{\mathrm{r}}$ of increasing size (in energy norm). The sequence shows how switching on a perturbation makes the QNMs migrate to a new branch (that actually closely follows a pseudospectrum contour line, compare with Fig. 5), in such a way that the instability starts appearing at highly damped QNMs and descends in the spectrum as the perturbation grows (unperturbed values, in red, are kept along the sequence for comparison). The top panel corresponds to the nonperturbed potential shown in Fig. 3, the second panel shows how a random perturbation of (energy) norm $\left\|\delta \tilde{V}_{\mathrm{r}}\right\|_{E}=10^{-16}$ already reaches the sixth QNM overtone, and, in the third panel, a perturbation with $\left\|\delta \tilde{V}_{\mathrm{r}}\right\|_{E}=10^{-8}$ already reaches the third overtone. This confirms the instability already detected in the pseudospectrum, indicating its high-frequency nature. Crucially, to reach the fundamental mode, a perturbation of the same order $O(1)$ as the variation of the eigenvalue is required, thus demonstrating the stability of the fundamental QNM in agreement with the pseudospectrum in Fig. 5. Right panel: sequence of QNM spectra for the Pöschl-Teller potential subject to a deterministic perturbation $\delta \tilde{V}_{\mathrm{d}} \sim \cos (2 \pi k x)$. The first panel again shows the unperturbed potential, whereas the second one shows that a low-frequency $(k=1)$ perturbation leaves the spectrum unperturbed, in spite of the $\left\|\delta \tilde{V}_{\mathrm{d}}\right\|_{E}=10^{-8}$ norm (compare with the random case with the same norm): This illustrates the harmless character of low-frequency perturbations. The third panel shows how keeping the norm of the perturbation but increasing its frequency indeed switches on the instability, confirming the high-frequency insight gained from random perturbations. The fourth panel shows how the instability increases with the frequency but less efficiently than with random perturbations of the same norm. 


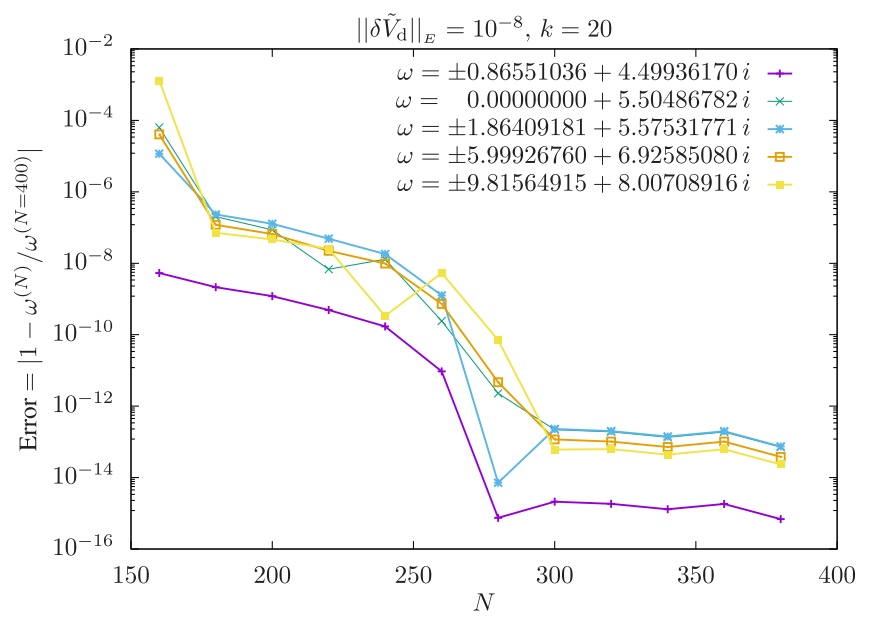

FIG. 8. Convergence test for five significant QNMs of the Pöschl-Teller potential perturbed under a deterministic highfrequency perturbation $\delta \tilde{V}_{\mathrm{d}}$ (cf. text). This behavior demonstrates that the large QNM "migrations" observed in Fig. 7 are not a numerical artifact, but actually, very small perturbations of the potential can result in large variations of the QNM spectrum, consistent with the pseudospectrum in Fig. 5.

the values in the stable self-adjoint case in Fig. 6. This highfrequency stability is then confirmed in the perturbation analysis. Indeed, Fig. 9 demonstrates the need for large perturbations in the operator in order to reach the fundamental QNM, namely, (random) perturbations with a size $\|\delta \tilde{V}\|_{E}$ of the same order as the induced variation in $\omega_{0}^{ \pm}$. This behavior is a manifestation of spectral stability.

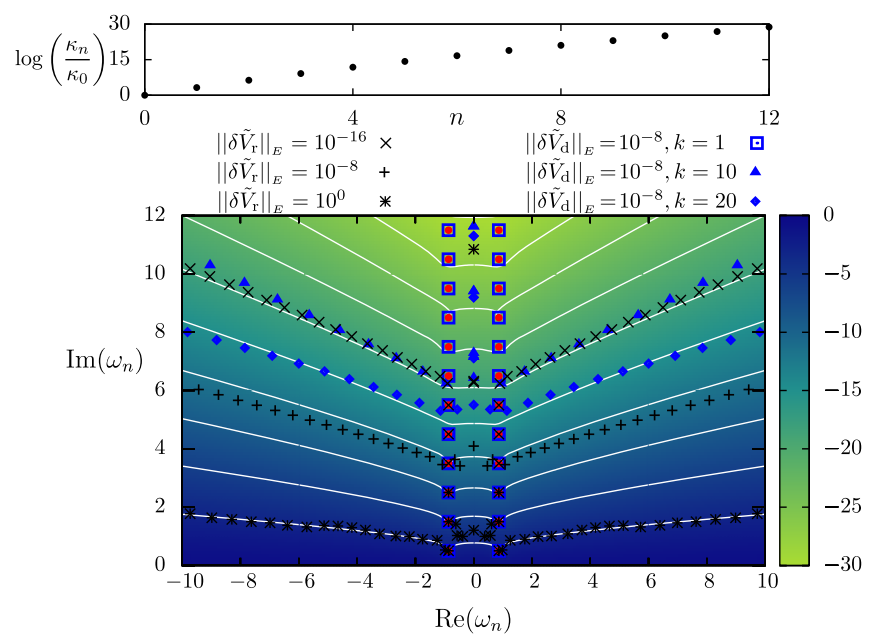

FIG. 9. QNM spectral instability of Pöschl-Teller potential. Combination of Figs. 3, 5, and 7, corresponding to three independent calculations, respectively: condition number ratios $\kappa_{n} / \kappa_{0}$ (top panel), pseudospectrum, and perturbed QNM spectra (bottom panel). The bottom panel demonstrates the high-frequency nature of the spectral instability, as well as the migration of Pöschl-Teller QNMs towards pseudospectrum contour lines under high-frequency perturbations.
The contrast between the high stability of $\omega_{0}^{ \pm}$and the instability of overtone resonances $\omega_{n \geq 1}^{ \pm}$has already been evoked in Sec. V B 2, when referring to the large condition number ratios $\kappa_{n} / \kappa_{0}$, in particular, referring to Bindel and Zworski's discussion in Refs. [99,100]. This high-frequency stability of the fundamental mode is in tension with the instability found by Nollert in Ref. [44] for the slowest-decaying mode for Schwarzschild spacetime. We will revisit this point in Sec. VID 3. For the time being, we simply emphasize that the observed stability relies critically on the faithful treatment of the asymptotic structure of the potential, which is built into the adopted hyperboloidal approach, allowing us to capture the long-range structure of the potential up to null infinity $\mathcal{I}^{+}$. It is only when we enforce a modification of the potential at large distances that the lowfrequency fundamental QNM is affected. This phenomenon is illustrated in Fig. 10 (see also Refs. [47,108]), corresponding to a Pöschl-Teller potential set to zero beyond a compact interval $\left[x_{\min }, x_{\max }\right]$ : Such a cut introduces high frequencies that migrate the overtones to the new branches, and, crucially, it alters the asymptotic structure so that the fundamental QNM is also modified. Such an "infrared" effect is, however, compatible with the spectral stability of the fundamental QNM since such a cut of the potential does not correspond to a small perturbation in $\delta L$.

\section{Regularization effect of random perturbations}

Before proceeding to the $\mathrm{BH}$ case, let us briefly comment on an apparently paradoxical phenomenon resulting from the interplay between random perturbations and the pseudospectrum. In contrast with what one might expect,

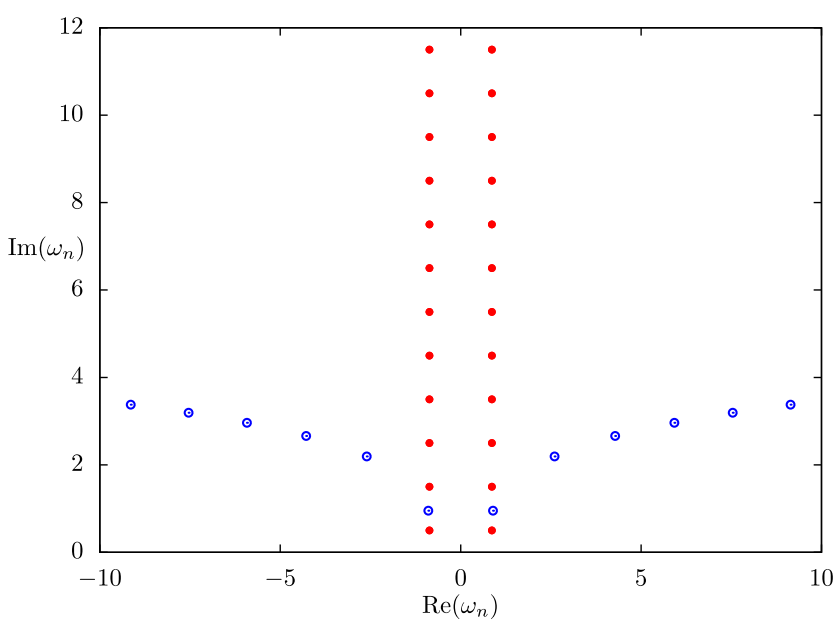

FIG. 10. QNMs of the Pöschl-Teller cut potential. Setting the Pöschl-Teller potential to zero outside an interval $\left[x_{\min }, x_{\max }\right]$ introduces high-frequency perturbations that make QNM overtones migrate towards pseudospectrum contour lines, as well as an "infrared" modification that alters the fundamental QNM frequency. Whereas the latter tends to the nonperturbed PöschlTeller value as $x_{\min } \rightarrow-\infty$ and $x_{\max } \rightarrow \infty$, QNM overtones always remain strongly perturbed. 
the addition of a random perturbation to a spectrally unstable operator $L$ does not worsen the regularity properties of $L$ but, on the contrary, it improves the analytical behavior of its resolvent $R_{L}(\omega)$ [9,109-118]. This is illustrated in Fig. 11, which shows a series of pseudospectra corresponding to random perturbations of the Pöschl-Teller potential with increasing $\left\|\delta \tilde{V}_{\mathrm{r}}\right\|_{E}$. In addition to the migration of QNM overtones towards pseudospectra contour lines, we observe two phenomena: (i) $\epsilon$-pseudospectra sets with $\epsilon>\left\|\delta \tilde{V}_{\mathrm{r}}\right\|_{E}$ are not affected by the perturbation, whereas (ii) the pseudospectrum structure for $\epsilon<\left\|\delta \tilde{V}_{\mathrm{r}}\right\|_{E}$ is smoothed into a flat pattern. As we have discussed in
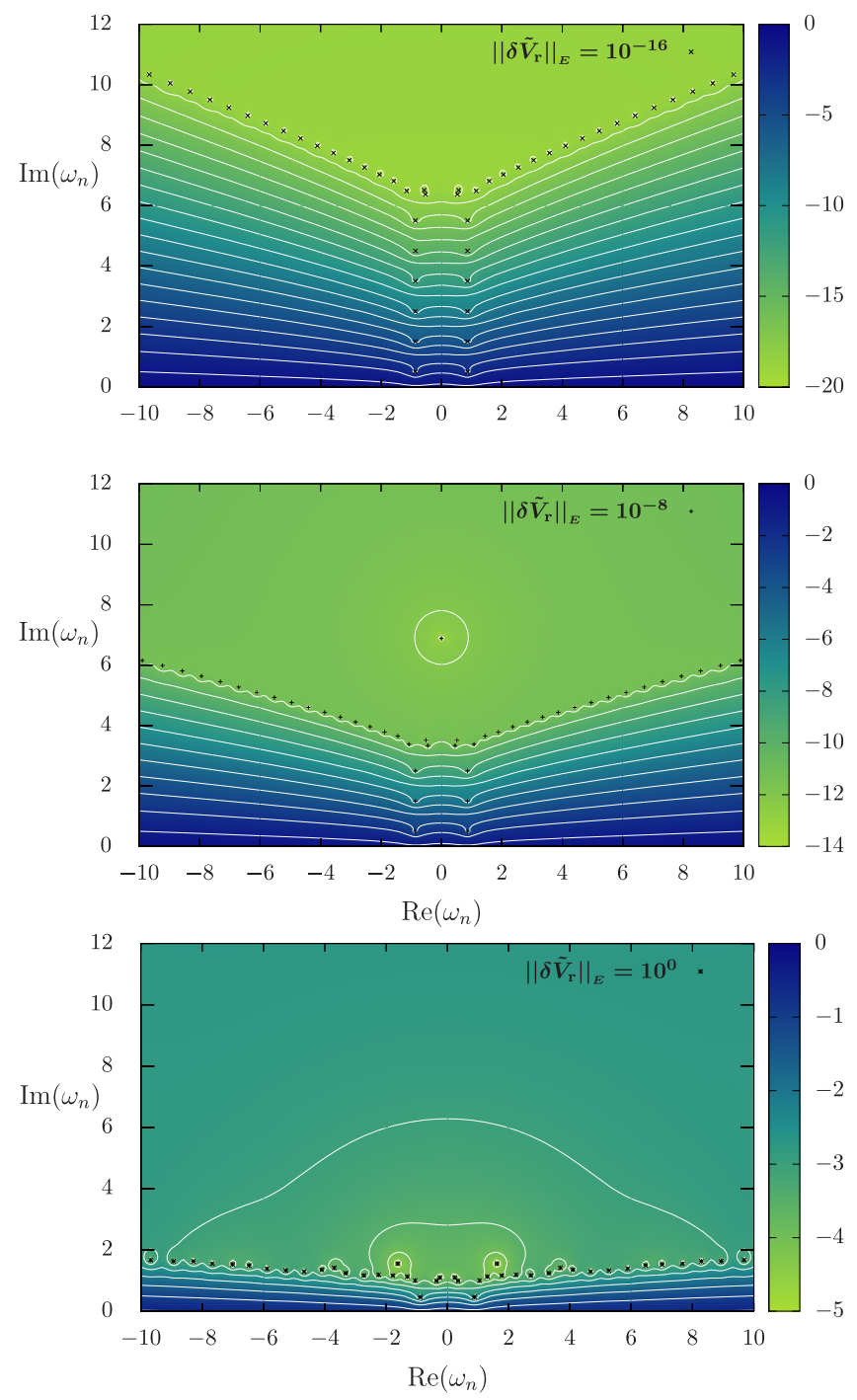

FIG. 11. Pseudospectra of the Pöschl-Teller potential under random perturbations $\delta \tilde{V}_{\mathrm{r}}$ of increasing norm, demonstrating the regularizing effect of random perturbations. Pseudospectra sets $\sigma^{\epsilon}$ bounded by the contour line reached by perturbed QNMs become flat, a signature of improved analytic behavior of the resolvent, as illustrated in Fig. 6. Pseudospectra sets not attained by the perturbation remain unchanged. Regularization of $R_{L+\delta L}(\omega)$ increases as $\left\|\delta \tilde{V}_{\mathrm{r}}\right\|_{E}$ grows.
Fig. 6, such flat pseudospectra patterns are the signature of spectral stability, a feature of regularity of the resolvent $R_{L}(\omega)$. The resulting improvement in the spectral stability of $L+\delta L$, as compared to $L$, is indeed consistent with the convergence properties of the respective QNM spectra, as illustrated by the contrast between the corresponding convergence tests in Figs. 8 and 4. In summary, random perturbations improve regularity, an intriguing effect seemingly intimately related to a Weyl law occurring in the large- $n$ asymptotics of QNMs [100,119], with suggestive physical implications in the QNM setting, e.g., in (semi) classical limits to smooth spacetimes from (random) structures at Planck scales.

\section{SCHWARZSCHILD QNM (IN)STABILITY}

We now address the physical $\mathrm{BH}$ case, namely, the stability of QNMs in Schwarzschild spacetime. Whereas the previous section has been devoted, to a large extent, to discussing some of the technical issues in QNM stability, the spirit in this section is to focus more on the physical implications, in particular, in the perspective of assessing the pioneering work in Refs. $[44,45]$.

\section{A. Hyperboloidal approach in Schwarzschild spacetime}

The attempt to implement the QNM stability analysis in the coordinate system employed for the Pöschl-Teller potential, namely, the Bizon-Mach chart (46), was unsuccessful because of the bad analytic behavior at null infinity of the Schwarzschild potential(s) in the corresponding coordinate $x$. Instead, we resort to the minimal gauge slicing $[75,76,120]$, devised to improve regularity in the Schwarzschild(-like) case.

We start by considering standard Schwarzschild $(t, r)$ coordinates in the line element (2), with $f(r)=$ $(1-2 M / r)$ and a BH horizon at $r=2 M$. Axial and polar Schwarzschild gravitational parities are described by the wave equation (4) with, respectively, Regge-Wheeler $V_{\ell}^{\mathrm{RW}, s}(r)$ and Zerilli $V_{\ell}^{\mathrm{Z}}(r)$ potentials $[12,13,82,121,122]$. Specifically, we have

$V_{\ell}^{\mathrm{RW}, s}(r)=\left(1-\frac{2 M}{r}\right)\left(\frac{\ell(\ell+1)}{r^{2}}+\left(1-s^{2}\right) \frac{2 M}{r^{3}}\right)$,

for the axial case, where $s=0,1,2$ correspond to the scalar, electromagnetic, and (linearized) gravitational cases, and

$$
\begin{aligned}
V_{\ell}^{\mathrm{Z}}(r)= & \left(1-\frac{2 M}{r}\right) \\
& \times\left(\frac{2 n^{2}(n+1) r^{3}+6 n^{2} M r^{2}+18 n M^{2} r+18 M^{3}}{r^{3}(n r+3 M)^{2}}\right),
\end{aligned}
$$


with

$$
n=\frac{(\ell-1)(\ell+2)}{2}
$$

for the polar case.

To construct horizon-penetrating coordinates reaching null infinity, one defines a height function $h$ in Eq. (6) by first considering an advanced time coordinate built on the rescaled tortoise coordinate $\bar{x}=r^{*} / \lambda$, with $r_{*}=r+2 M \ln (r / 2 M-1)$, so that the BH horizon is at $\bar{x} \rightarrow-\infty$; one then enforces a deformation of the Cauchy slicing into a hyperboloidal one through the choice of a minimal gauge, prescribed under the guideline of preserving a good analytic behavior at $\mathcal{I}^{+}$. In a second stage, the function $g$ in Eq. (6) implementing the compactification along hyperboloidal slices is implicitly determined by [note that instead of $x$ in Eq. (6), we rather use $\sigma$ for the spatial coordinate, so as to keep the standard usage in Refs. [75,76,120] ]

$$
r=\frac{2 M}{\sigma} .
$$

Choosing $\lambda=4 M$ in the rescaling $\bar{x}=r^{*} / \lambda$ of Eq. (5), the steps above result in (see details in Refs. $[75,76,120]$ ) the minimal-gauge hyperboloidal coordinates for the transformation (6),

$$
\left\{\begin{array}{l}
\bar{t}=\tau-\frac{1}{2}\left(\ln \sigma+\ln (1-\sigma)-\frac{1}{\sigma}\right) \\
\bar{x}=\frac{1}{2}\left(\frac{1}{\sigma}+\ln (1-\sigma)-\ln \sigma\right),
\end{array}\right.
$$

which, upon the addition of the $\mathrm{BH}$ horizon and $\mathcal{I}^{+}$points, maps $\bar{x} \in[-\infty, \infty]$ to the compact interval $\sigma \in[a, b]=$ $[0,1]$, with the BH horizon at $\sigma=1$ and future null infinity at $\sigma=0$.

Implementing transformation (69) in the first-order reduction in time in Eqs. (8) and (9), we get, for $w(\sigma)$, $p(\sigma), q_{\ell}(\sigma)$ (now explicitly depending on $\ell$ ), and $\gamma(\sigma)$ in Eq. (12),

$$
\begin{array}{cc}
w(\sigma)=2(1+\sigma), & p(\sigma)=2 \sigma^{2}(1-\sigma), \\
q_{\ell}(\sigma)=\frac{(4 M)^{2} V_{\ell}}{2 \sigma^{2}(1-\sigma)}, & \gamma(\sigma)=1-2 \sigma^{2},
\end{array}
$$

leading to the $L_{1}$ and $L_{2}$ operators building $L$ in Eq. (10),

$$
\begin{aligned}
& L_{1}=\frac{1}{2(1+\sigma)}\left[\partial_{\sigma}\left(2 \sigma^{2}(1-\sigma) \partial_{\sigma}\right)-\tilde{V}_{\ell}\right], \\
& L_{2}=\frac{1}{2(1+\sigma)}\left(2\left(1-2 \sigma^{2}\right) \partial_{\sigma}-4 \sigma\right),
\end{aligned}
$$

where the rescaled potential $\tilde{V}_{\ell}(\sigma):=q_{\ell}(\sigma)$ results, in the respective axial and polar cases, in the explicit expressions

$$
\begin{aligned}
\tilde{V}_{\ell}^{\mathrm{RW}, s} & =2\left(\ell(\ell+1)+\left(1-s^{2}\right) \sigma\right), \\
\tilde{V}_{\ell}^{\mathrm{Z}} & =2\left(\sigma+\frac{2 n}{3}\left(1+4 n \frac{3+2 n}{(2 n+3 \sigma)^{2}}\right)\right) .
\end{aligned}
$$

Finally, from Eqs. (70) and (22), the energy scalar product is

$$
\begin{aligned}
\left\langle u_{1}, u_{2}\right\rangle_{E}= & \left\langle\left(\begin{array}{c}
\phi_{1} \\
\psi_{1}
\end{array}\right),\left(\begin{array}{c}
\phi_{2} \\
\psi_{2}
\end{array}\right)\right\rangle_{E} \\
= & \int_{0}^{1}\left((1+\sigma) \bar{\psi}_{1} \psi_{2}+\sigma^{2}(1-\sigma) \partial_{x} \bar{\phi}_{1} \partial_{x} \phi_{2}\right. \\
& \left.+\frac{\tilde{V}_{\ell}}{2} \bar{\phi}_{1} \phi_{2}\right) d \sigma,
\end{aligned}
$$

where the weight $\tilde{V}_{\ell}$ is fixed by Eq. (72) for each polarization.

\section{B. Schwarzschild QNM spectrum}

As discussed in Sec. II C 1, outgoing boundary conditions have been translated into regularity conditions on eigenfunctions. Specifically, as we have seen in the PöschlTeller case, the operator $L_{1}$ in Eq. (71) is a singular SturmLioville operator; namely, the function $p(\sigma)=2 \sigma^{2}(1-\sigma)$ vanishes at the boundaries of the interval $[a, b]=[0,1]$ consistently with Eq. (16). This result translates into the fact that no boundary conditions can be imposed if enough regularity is required.

However, there is a key difference between the PöschlTeller and the BH case: Whereas in the Pöschl-Teller case the function $p(x)=(1-x)(1+x)$ vanishes linearly at the boundaries, and therefore $x= \pm 1$ are regular singular points, in the Schwarzschild case this is true for $\sigma=1$ (BH horizon) but not for $\sigma=0\left(\mathcal{I}^{+}\right)$because of the quadratic $\sigma^{2}$ term. Null infinity is then an irregular singular point, which is the counterpart, in our compactified hyperboloidal formulation, of the power-law decay of Schwarzschild potentials responsible for the branch cut in the Green function of Eq. (4), with its associated "tails" in late decays of scattered fields. In the context of our spectral problem for the operator $L$, this translates into the appearance of a (branch-cut) continuous part in the spectrum. This has an important impact on the numerical approach since the continuous branch cut is realized in terms of actual eigenvalues of the discretized approximates $L^{N}$. Such eigenvalues are not QNMs and can indeed be unambiguously identified, but their presence has to be taken into account when performing the spectral stability analysis, which becomes a more delicate problem than in the Pöschl-Teller case. In this context, the latter becomes a crucial benchmark to guide the analysis in the $\mathrm{BH}$ case.

The Schwarzschild (gravitational) QNM spectrum (for $\ell=2$ ) is shown in Fig. 12, which presents the result of the numerical calculation of the spectrum of the $L$ operator defined by Eq. (70). This result is obtained either for the 

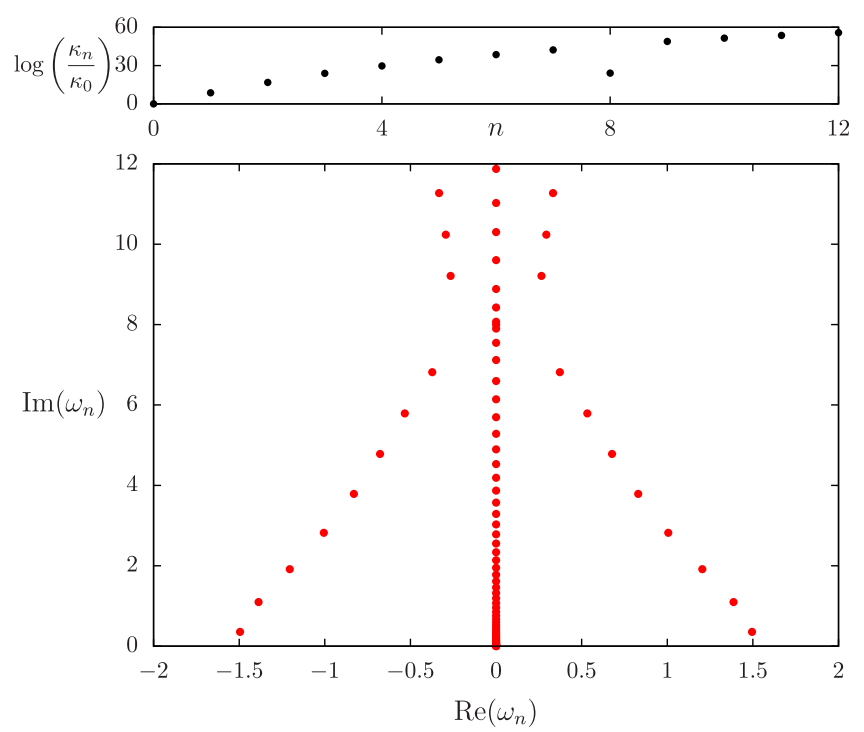

FIG. 12. Schwarzschild QNM problem. Bottom panel: QNMs for the $\ell=2$ axial and polar gravitational modes of Schwarzschild spacetime, corresponding, respectively, to the (isospectral) Regge-Wheeler and Zerilli potentials (eigenvalues along the imaginary upper half line are the numerical counterpart of the Schwarzschild branch cut, but also the algebraically special QNM $\omega_{n=8}$; see Ref. [75] for a discussion of this). Note the normalization $4 M \omega_{n}$, consistent with $\lambda=4 M$ after Eq. (68). Top panel: condition numbers $\kappa_{n}$ normalized to the condition number $\kappa_{0}$ of the fundamental QNM. Note the relative enhanced stability of the algebraically special QNM.

Regge-Wheeler or the Zerilli rescaled potentials in Eq. (72), corresponding, respectively, to potentials (65) and (66), thus providing a crucial internal consistency check for the analytical and numerical construction since both potentials are known to be QNM isospectral (see below in Sec. VI D 2). The branch-cut structure is apparent in the eigenvalues along the upper imaginary axis. Such branch-cut points can be easily distinguished from the special QNM corresponding to $\omega_{n=8}$, also in the imaginary axis, simply by changing the resolution: Branch points move randomly along the vertical axis, whereas $\omega_{n=8}$ stays at the same frequency (see Sec. VID 1 for a more systematic approach to establish the nonbranch nature of $\omega_{n=8}$, where we consider highfrequency perturbations to QNMs). Moreover, eigenfunctions associated with algebraically special modes are polynomials, as shown in the detailed studies of these modes for Schwarzschild and Kerr spacetimes in Refs. [75,123].

Because of the lack of an exact expression for the Schwarzschild QNMs, one must compare the obtained values against those available in the literature via alternative approaches-see, for instance, Refs. [13-16,124-127]. An estimative for the errors when QNMs are calculated with the methods from this work is found in Ref. [120]. From a practical perspective, and regardless of the numerical methods, it is well known that the difficulty to accurately calculate numerically a given QNM overtone $\omega_{n}^{ \pm}$increases significantly with $n$. For instance, convergence and machine precision issues similar to the ones commented above are reported in Refs. [128-130], with a control of the internal roundoff accuracy being required. Alternatively, iterative algorithms such as Leaver's continued fraction method [131] require an initial seed relatively near a given QNM, which must be carefully adapted when dealing with the overtones [132]. The bottom line is that the calculation of BH QNM overtones is a challenging and very delicate issue.

In our understanding, the latter challenge is not a numerical hindrance but the consequence of a structural feature of the underlying analytical problem, namely, the spectral instability of the Schwarzschild QNM problem. This is manifested already at the present stage of analysis, namely, the calculation of QNM frequencies of nonperturbed Schwarzschild's potential, in the eigenvalue condition numbers $\kappa_{n}$ 's shown in the top panel of Fig. 12: We again encounter the pattern found in the Pöschl-Teller case, cf. Fig. 3, with a growth of the spectral instability as the damping increases, with the notable anomaly of an enhanced stability for the algebraically special QNM frequency, with $n=8$. We devote the rest of the section to exploring this spectral instability with the tools employed for the Pöschl-Teller potential.

\section{Schwarzschild pseudospectrum}

The pseudospectrum of Schwarzschild spacetime is presented in Fig. 13. As illustrated in the Pöschl-Teller potential, the pseudospectrum provides a systematic and global tool to address QNM spectral instability, already at the level of the unperturbed potential. In a topographic map of the analytic structure of the resolvent, regions associated with small $\epsilon$-pseudospectra (light green) correspond to strong spectral instability, whereas regions with large $\epsilon$ [namely, $O(\epsilon) \sim 1$, dark blue] indicate spectral stability. The superposition of the QNM spectrum shows the respective spectral stability of QNM frequencies.

We can draw the following conclusions from Fig. 13:

(i) The Schwarzschild pseudospectrum indicates a strong instability of QNM overtones, an instability that grows quickly with the damping. White-line boundaries corresponding to $\epsilon$-pseudospectra with very small $\epsilon$ 's extend in large regions of the complex plane, which is compatible with the results in Ref. [44], providing a rationale_-already at the level of the unperturbed potential-for the QNM overtone instability discovered by Nollert.

(ii) The slowest-decaying QNM is spectrally stable. Figure 13 tells us that changing the fundamental QNM frequency requires perturbations in the operator of order $\|\delta L\|_{E} \sim 1$, which corresponds to spectral stability and is in tension with the results in Ref. [44], where the fundamental QNM is found to be unstable. We will address this point below. 


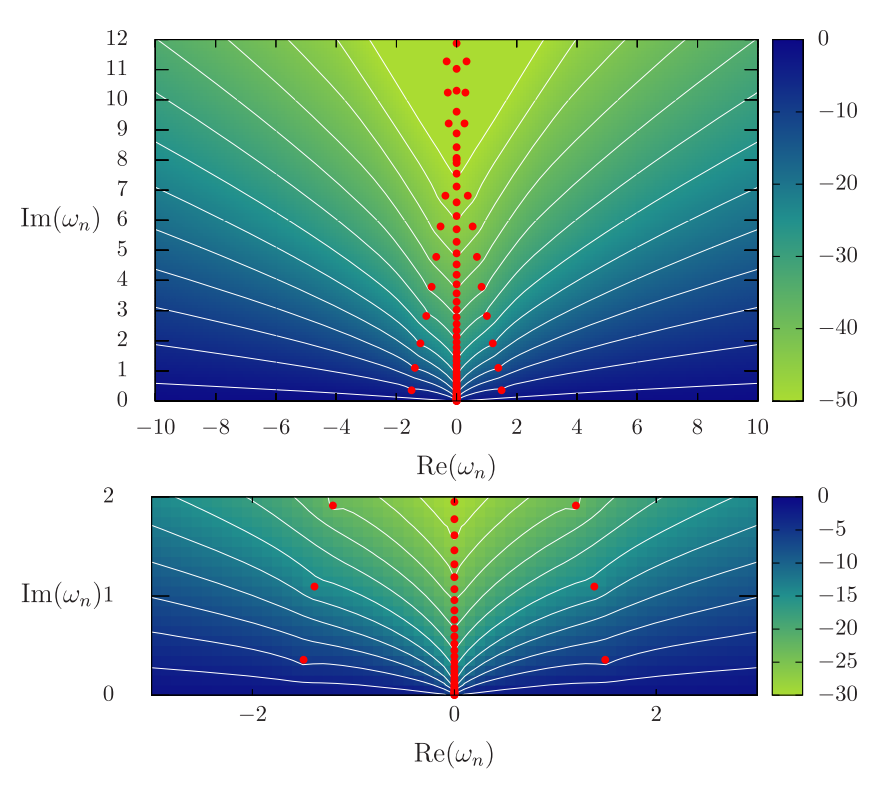

FIG. 13. Top panel: pseudospectrum of Schwarzschild spacetime ( $\ell=2$ gravitational modes, from Regger-Wheeler potential, and similar for Zerilli). Again, QNM frequencies (red circles) from Fig. 12 are superimposed for reference on their (in)stability. The pattern of $\epsilon$-pseudospectra sets $\sigma^{\epsilon}$ is qualitatively similar to the Pöschl-Teller one (cf. Fig. 5), though presenting an enhanced spectral instability indicated by the smaller $\epsilon$ values of $\epsilon$ pseudospectra contour lines (cf. range in color log scale for $\log _{10} \epsilon$ in Fig. 5). Bottom panel: zoom into the region around the fundamental QNM and first overtones.

(iii) Schwarzschild and Pöschl-Teller potentials show qualitatively the same pseudospectrum pattern, with large "green regions" producing patterns in stark contrast with the flat self-adjoint case. On the one hand, this shared behavior reinforces the usage of the Pöschl-Teller potential as a convenient guideline for understanding the stability structure of $\mathrm{BH}$ QNMs; on the other hand, it points towards an instability mechanism independent, at least in some measure, on certain details of the potential.

We can conclude that Fig. 13 demonstrates-at the level of the unperturbed operator - the main features of the stability structure of the BH QNM spectrum, namely, the QNM overtone instability and the stability of the fundamental QNM. However, the pseudospectrum does not inform us about the particular type of perturbations that trigger the instabilities, which is addressed in the following subsection.

\section{Perturbations of the Schwarzschild potential}

Once the Schwarzschild pseudospectrum, together with the condition numbers $\kappa_{n}$, have presented evidence of QNM spectral instability at the level of the unperturbed operator, we address the question about the actual physical character of perturbations triggering such instabilities.

\section{Ultraviolet instability of BH QNM overtones}

The qualitative agreement between Pöschl-Teller and Schwarzschild pseudospectra, cf. Figs. 5 and 13, together with the experience gained in the study of Pöschl-Teller perturbations regarding the high-frequency instability of all QNM overtones and the stability of the fundamental QNM, guide our steps in the analysis of the $\mathrm{BH}$ setting.

Random perturbations: spoils from the branch cut.-The presence of a branch cut in the Schwarzschild spectrum, discussed in Sec. VIB, translates into a methodological subtlety when considering random perturbations in the $\mathrm{BH}$ case, as compared with the Pöschl-Teller one. The difficulty stems from the fact that not only the QNM eigenvalues, but also the eigenvalues associated with the discretized version of the branch cut, are sensitive to random perturbations $\delta \tilde{V}_{\mathrm{r}}$ of the potential. As a consequence, the possible contamination from eigenvalues from the branch cut complicates the analysis of the impact of random perturbations on QNM frequencies, which is an artifact of our particular numerical approach and not a problem of the differential operator itself; however, it limits our capability to assess the triggering by random perturbations of the QNM migration to new branches, which was observed in the Pöschl-Teller case (cf. left column of Fig. 7). Other tools, either numerical refinements and/or analytical methodologies, are required to address this specific issue in the Schwarzschild case.

In spite of this, random perturbations are still useful in our $\mathrm{BH}$ discussion. An illustrative example is the study of the stability of the algebraically special Schwarzschild QNM $\omega_{n=8}$. Whereas random perturbations move branch-cut eigenvalues away from the imaginary axis, the algebraically special QNM stays stable. This methodology provides a powerful and efficient tool to probe the physicality of specific eigenvalues in very general settings (cf. e.g., Fig. 4 in Ref. [133]).

Deterministic perturbations.-Given the limitations for random $\delta \tilde{V}_{\mathrm{r}}$ 's, in the present study we have focused on the class of deterministic perturbations to the potential $\delta \tilde{V}_{\mathrm{d}}$ provided by Eq. (63). Crucially, such perturbations do not perturb the branch eigenvalues as (much as) random $\delta \tilde{V}_{\mathrm{r}}$ do, thus bypassing the associated spectral instability contamination. Despite their simplicity, they provide a good toy model to explore the effects of astrophysically motivated perturbations (assessment of long-range and low-frequency versus small-scale and high-frequency perturbations), as well as those arising from generic approaches to quantum gravity (small-scale and highfrequency effective fluctuations). Therefore, they are conveniently suited to address these instability issues.

The left column in Fig. 14 depicts (with $\left\|\delta \tilde{V}_{\mathrm{d}}\right\|_{E} \sim 10^{-8}$ ) the stability of the first overtones against low-frequency perturbations ( $k=1$, top-left panel), in contrast with the instability resulting from high-frequency perturbations ( $k=20$, bottom-left panel). Along this line, the right 

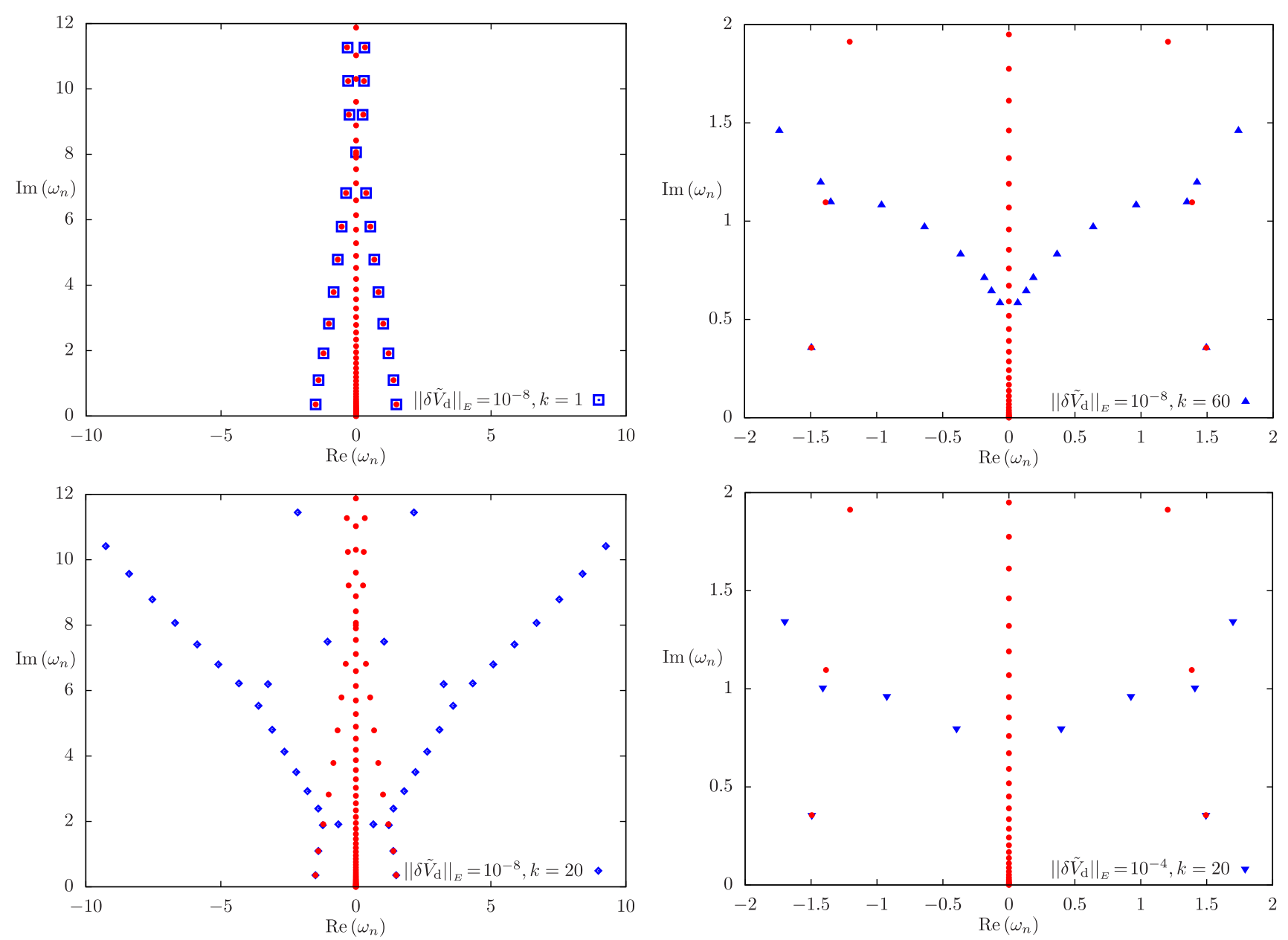

FIG. 14. QNM spectra for deterministic perturbations $\delta \tilde{V}_{\mathrm{d}}$ of Schwarzschild $\ell=2$ gravitational modes (here Regge-Wheeler, similar behaviour for Zerilli, cf. Fig. 15), superimposed over the unperturbed values (red). Left column: stability under low-frequency perturbation (top panel) versus high-frequency instability of QNM overtones (bottom panel). Right column: zoom into the first QNM overtones, showing the instability of the first overtone by increasing (i) the frequency of the perturbation (top panel) and (ii) the (energy) norm of the perturbation (bottom panel).

column in Fig. 14 zooms in to study the very first overtones, which are paramount for the incipient field of black hole spectroscopy. Assessing the (in)stability of the very first overtones is therefore crucial for current research programs in gravitational astronomy. It becomes apparent that the first overtones, including the very first overtone, are indeed affected without any extraordinary or fine-tuned perturbations $\delta \tilde{V}_{\mathrm{d}}$. In particular, and taking the left column as a reference, the first overtone is reached: either by considering (i) a slightly more intense perturbation $\left(\left\|\delta \tilde{V}_{\mathrm{d}}\right\|_{E} \sim 10^{-4}, k=20\right)$ or (ii) perturbations with sufficiently high frequency $\left(\left\|\delta \tilde{V}_{\mathrm{d}}\right\|_{E} \sim 10^{-8}, k=60\right)$.

From this perturbation analysis of the $\mathrm{BH}$ potential, we make the following conclusions: (i) All QNM overtones are ultraviolet unstable, as in the Pöschl-Teller case, with the instability reaching the first overtone for sufficiently high frequency; (ii) QNMs are stable under low-frequency perturbations, illustrating that spectral instability does not mean instability under any perturbation, in particular, long-wave perturbations not affecting the QNM spectrum; (iii) the slowest-decaying QNM is ultraviolet stable, a result that is in tension with the instability of the fundamental QNM found in Ref. [44]. We revisit this point in Sec. VID 3 below.

\section{Isospectrality loss: Axial versus polar spectral instability}

Regge-Wheeler and Zerilli potentials for axial and polar perturbations are known to be isospectral in the QNM spectrum (cf. Refs. [12,134-136]; see also Ref. [82]). In particular, Chandrasekhar identified (cf. point 28 in Ref. [12]) a necessary condition for two (one-dimensional) potentials $V_{1}(\bar{x})$ and $V_{2}(\bar{x})$, with $\left.\bar{x} \in\right]-\infty, \infty[$ as it is the case for the rescaled tortoise coordinate, to have the same transmission amplitude and present the same QNM spectrum. Specifically, both potentials must render the same values when evaluating an infinite hierarchy of integrals, 


$$
C_{n}=\int_{-\infty}^{\infty} v_{n}(\bar{x}) d \bar{x}
$$

with

$$
\begin{aligned}
& v_{1}=V, \quad v_{3}=2 V^{3}+V^{\prime 2}, \\
& v_{5}=5 V^{4}+10 V V^{\prime 2}+V^{\prime \prime 2}, \quad v_{2 n+1}=\ldots
\end{aligned}
$$

These quantities are the conserved quantities of the Korteweg-de Vries equation, and they connect the Schwarzschild QNM isospectrality problem to integrability theory through the inverse scattering transform of GelfandLevitan-Marchenko (GLM) theory (cf. Ref. [137]; see Ref. [136] for an alternative approach in terms of Darboux transformations).

The key point for our spectral stability analysis of $L$ is that axial and polar QNM isospectrality is the consequence of a subtle and delicate integrability property of stationary $\mathrm{BH}$ solutions, so we do not expect it to be robust under generic perturbations of $V$. In particular, given the nonlinear dependence in $V$ of the conserved quantities $C_{n}$ in Eq. (74), we would expect changes in $V$ under either random $\delta \tilde{V}_{\mathrm{r}}$ or deterministic $\delta \tilde{V}_{\mathrm{d}}$ perturbations to render different values of $C_{n}$, therefore resulting in a loss of QNM isospectrality. Figure 15 confirms this expectation: Whereas the fundamental QNM mode remains stable under high-frequency perturbations, isospectrality is broken for the overtones with a slight, but systematic, enhanced damping in the axial case. Other mechanisms for $\mathrm{BH}$ isospectrality loss have been envisaged, e.g., in the study of the imprints of modified gravity theories [50], whereas ultraviolet QNM overtone instability would provide a possible mechanism inside general relativity. In summary, isospectrality loss provides an interesting probe into QNM instability, with potential observable consequences, and it will be the subject of a specifically devoted study elsewhere.

\section{Infrared instability of the fundamental QNM}

Both the pseudospectrum and the explicit perturbations of the potential indicate a strong spectral stability of the slowest-decaying Schwarzschild QNM, which is tension with the results in Refs. [44,45], where the instability affects the whole QNM spectrum, including the slowestdecaying QNM. This point is fundamental to establish since it directly impacts the dominating frequency in the late $\mathrm{BH}$ ringdown signal.

In our understanding, and as was the case of the PöschlTeller potential discussed in Sec. V D 4, the instability of the fundamental QNM frequency found by Ref. [44] is an artifact of the implemented perturbations, namely, steplike approximations to the Schwarzschild potential (in particular, Regge-Wheeler, but the same applies for Zerilli) that modify the potential at large distances. Specifically, $V_{\ell}$ is set to zero beyond $\left[x_{\min }, x_{\max }\right]$, fundamentally altering the

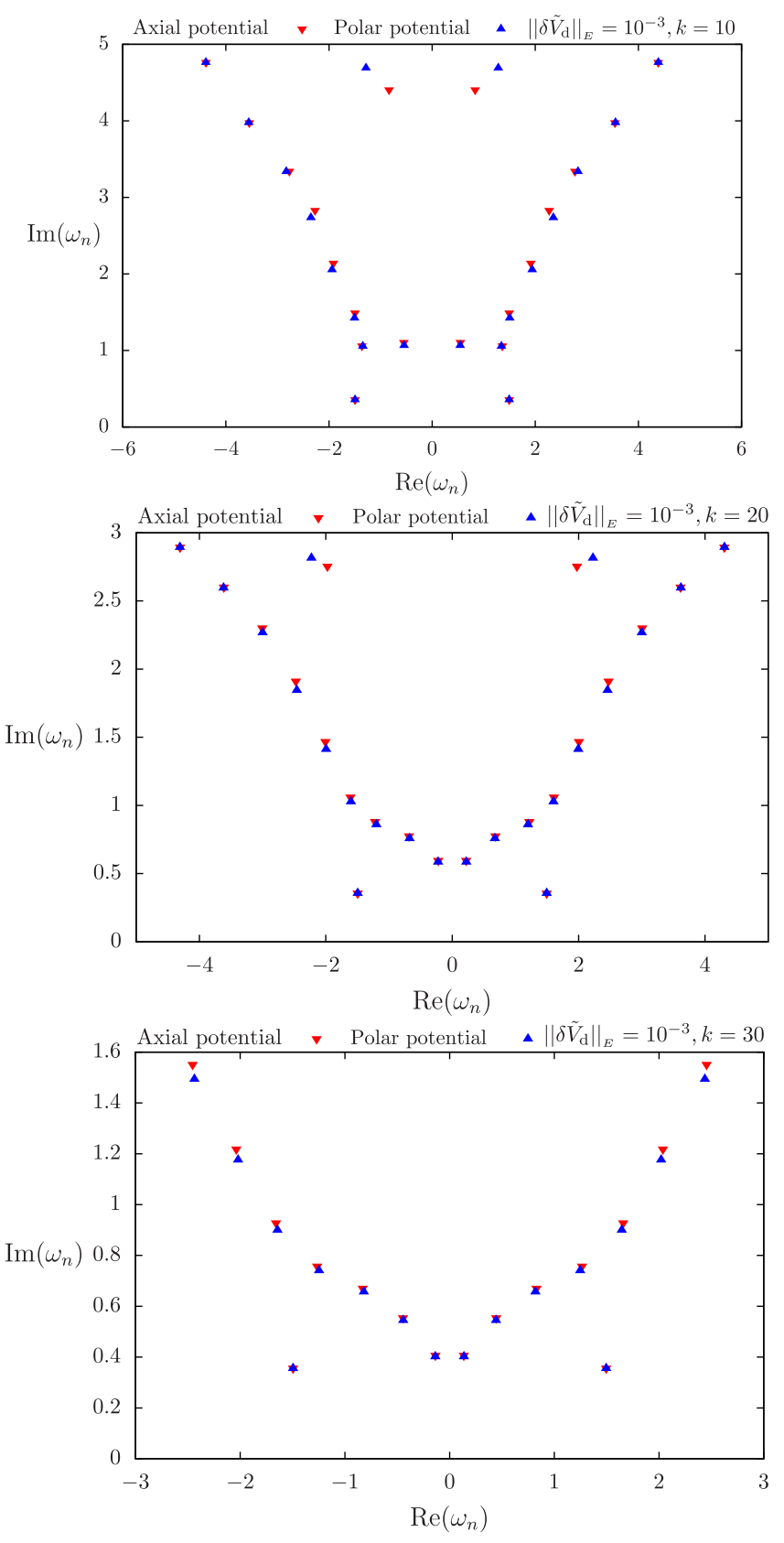

FIG. 15. Loss of QNM isospectrality in Schwarzschild spacetime, under high-frequency perturbations. The sequence of figures shows a zoom into the perturbation of lowest $\ell=2$ axial and polar QNM overtones (the branch cut has been removed), with $\delta \tilde{V}_{\mathrm{d}}$ fixed to a value reaching the first overtone, and then increasing the frequency. The breaking of axial and polar isospectrality is demonstrated, with perturbed axial overtones slightly more damped than polar perturbed counterparts, though both are over the same perturbed QNM branches (actually tracking the pseudospectra contour lines, cf. Fig. 17 below). The fundamental QNM remains unchanged, consistently with its stability, so the dominating ringdown frequency remains "isospectral."

long-range nature of the Schwarzschild potential that is then of compact support. What we observe in Fig. 14 is that using a faithful treatment of the asymptotic structure at 
infinity through the compactified hyperboloidal approach maintains spectral stability.

To test this idea (cf. also the recent work [47], as well as Ref. [108]), as we did in the Pöschl-Teller case, we have implemented a "cut Schwarzschild" potential in our hyperboloidal approach, setting the potential to zero from a given distance (both towards null infinity and the BH horizon). The result is shown in Fig. 16, showing a similar qualitative behavior to the Pöschl-Teller case in Fig. 10. Overtones are strongly perturbed into the QNM branches already observed in Fig. 14, consistently with the high frequencies introduced by the Heaviside cut. But, crucially, now the fundamental QNM is indeed also modified, in contrast with its stable behavior under high-frequency perturbations. This reinforces the understanding of this effect as a consequence of the "suppression" of the large-scale asymptotics of the potential [138]. However, the observed modification of the fundamental QNM frequency is not as dramatic as the one in Ref. [44]. We do not have a good explanation for this, but it may relate to the fact that the analysis in Refs. [44,45] deals directly with Eq. (4), in particular, in the setting of a Cauchy slicing getting to spatial infinity $i^{0}$. Such an asymptotic framework may be more sensitive to the modification of the potential than the hyperboloidal one, related to null infinity $\mathcal{I}^{+}$. In this setting, and lacking a better expression, we refer to this effect as an infrared instability of the fundamental QNM.

Enforcing the compact support nature of $V$ is naturally motivated in physical contexts such as optical cavities and will be studied systematically in such settings [108]. In gravitation, the physicality of such an effect is more difficult to assess since gravity is a long-range interaction that, in contrast to the electromagnetic one, is not screened.

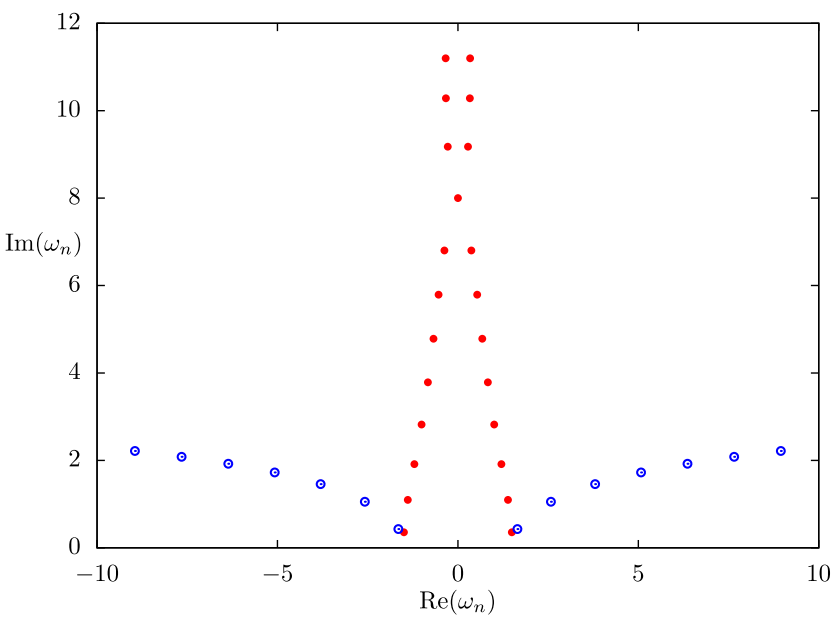

FIG. 16. Infrared modification of the Schwarzschild fundamental QNM. As in the Pöschl-Teller case, cutting the Schwarzschild potential ( $\ell=2$, either Regge-Wheeler or Zerilli) outside a compact interval $\left[x_{\min }, x_{\max }\right]$ modifies the fundamental QNM, thus accounting for its instability as found in Ref. [44]. All QNM overtones are strongly perturbed because of the high frequencies in the Heaviside cut, whereas (only) the fundamental QNM is recovered as $x_{\min }, x_{\max } \rightarrow \mp \infty$.
In any case, insofar as a pertinent gravitational scenario may be envisaged for such a cut potential, then the infrared instability shown for the first time in Ref. [44] would constitute a physical effect.

\section{E. Nollert-Price BH QNM branches: Instability and universality}

We revisit the results in Refs. [44,45] (see also Refs. [46,47]), under the light of the elements introduced for the study of QNM spectral stability. Figure 2 in Ref. [44] presents the migration of Schwarzschild QNMs to new branches, as a result of perturbing the (Regge-Wheeler) Schwarzschild potential with a steplike approximation with an increasing number $N_{\mathrm{st}}$ of steps (cf. Fig. 1 in Ref. [44]). A salient feature of Nollert's Fig. 2, further analyzed with Price in Ref. [45], is that the new QNM branches are distributed in a perfectly structured family of curves in the complex plane, unbounded in the real part of the frequency, that "move down" in the complex plane as $N_{\text {st }}$ (i.e., the frequency in the perturbation) increases [139]. A comparison with Schwarzschild's pseudospectrum in our Fig. 13 shows two remarkable features: (i) The pattern of the new branches found and studied by Nollert and Price is qualitatively similar to the contour lines of $\epsilon$-pseudospectra, and (ii) the effect of increasing the frequency perturbation indeed corresponds to an increment in the $\epsilon$ of the corresponding contour line (namely, the energy norm of the perturbation that, as a $H^{1}$ norm, includes the frequency). In other words, Nollert and Price's BH QNM branches indeed seem to be closely related to $\epsilon$-pseudospectrum contour lines.

In order to test this picture, we use our perturbation analysis from Sec. VID. Figure 17 presents the superposition of perturbed QNM spectra in Fig. 14 onto the Schwarzschild pseudospectrum in Fig. 13. As in the PöschlTeller case, perturbed QNMs closely track $\epsilon$-pseudospectra lines, demonstrating the insight gained above on Nollert's QNM instability by using the pseudospectrum: NollertPrice QNM branches are identified as actual probes into the analytical structure of the nonperturbed wave operator. Moreover, the correlation of $\epsilon$-contour lines with the size and frequency of the perturbations endows the pseudospectrum not only with an explicative but also with a predictive power, as a tool to calibrate the relation between spacetime perturbations and QNM frequency changes. The conceptual frame encoded in Fig. 17 is, in our understanding, the main contribution in this work.

\section{QNM structural stability, universality, and asymptotic analysis}

Building on Nollert and Price's work, our analysis strongly suggests that BH QNM overtones are indeed structurally unstable under high-frequency perturbations: BH QNM branches migrate to a qualitatively different class of QNM branches. Noticeably and in contrast with this, the pseudospectrum analysis combined with the perturbation tools also 


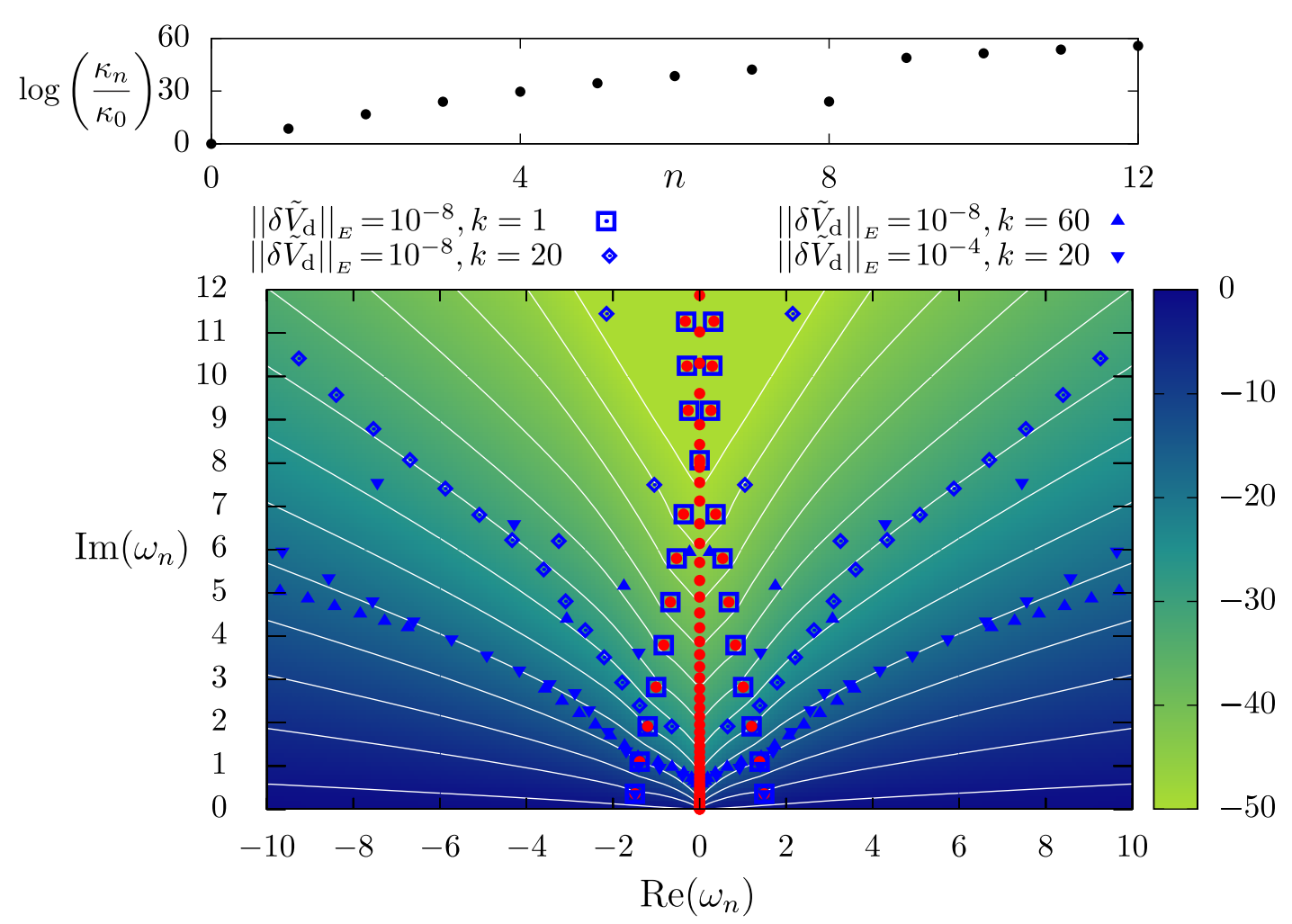

FIG. 17. Gravitational QNM spectral (in)stability in Schwarzschild spacetime (here, $\ell=2$ axial case corresponding to the ReggeWheeler potential, same behaviour for polar modes with Zerilli potential). The figure shows the superposition of the pseudospectrum of Fig. 13, perturbed QNM spectra in Fig. 14, together with exact QNMs and condition numbers $\kappa_{n}$ from Fig. 12. Employed norms follow from the energy scalar product in Eq. (73), i.e., energy defines "big" and "small". The pseudospectrum pattern, with $\epsilon$-pseudospectra sets with small $\epsilon$ extending into large regions of the complex plane, indicates spectral instability of QNM overtones, consistently with the fastly growing $\kappa_{n}$ 's. Perturbations in the potential demonstrate the high-frequency (ultraviolet) instability of all overtones and their stability under low-frequency perturbations. Both pseudospectrum and perturbations in the potential show the ultraviolet stability of the fundamental QNM. Ultraviolet instability induce QNM overtones to migrate towards $\epsilon$-pseudospectra contour lines, a pattern consistent with "Nollert-Price QNM branches" [44,45] here illustrated up to the lowest overtone. Universality of this pattern is further supported by comparison with the Pöschl-Teller potential in Fig. 9.

suggests that the new class of Nollert-Price BH QNM branches presents structural stability features pointing to a kind of universality in the QNM overtone migration pattern.

Universality in the high-frequency perturbations.-The QNM migration pattern seems to be independent of the detailed nature of the high-frequency perturbation in the Schwarzschild potential. First, such universality is manifested by a similar QNM perturbation pattern produced by very different perturbations: steplike perturbations in Ref. [44], the sinusoidal deterministic ones shown in Fig. 17, and also random perturbations (not presented here because of blurring issues, which are a consequence of the branch-cut contamination). Second, the new branches closely follow the pseudospectra contour lines, a key point in this universality discussion since it is completely prior to and independent of perturbations.

Universality in the potential.-Perhaps more importantly, universality seems to go beyond the insensitivity to the nature of the perturbation: It seems to be shared by a whole class of potentials. First, the same pattern of perturbed branches is found in the Pöschl-Teller potential, cf. Fig. 9. More dramatically, Nollert and Price's analysis in Ref. [45] is particularly illuminating in this respect. They considered a toy model capturing the effect of a (Dirac-delta) highfrequency perturbation on a BH-like potential, referred to as the truncated dipole potential (TDP), that contains only two QNMs. Adding the singular (high-frequency) "spike" creates an infinite number of QNMs, again following a QNM branch pattern compatible with our pseudospectra contour lines (cf. Fig. 5 in Ref. [45] and see below).

But more noteworthy, and again noticed by Nollert [44], beyond the $\mathrm{BH}$ setting, the new BH QNM branches are strikingly similar to (curvature) $w$ modes in neutron-star QNMs (cf. e.g., Fig. 3 in Ref. [13] and the systematic study in Ref. [140]). This result is remarkable, suggesting that exact but unstable BH QNMs migrate to perturbed but stable QNM branches whose qualitative pattern may be shared by generic compact objects [141]. 
Asymptotic analysis and universality.-How does one systematically address a possible universality in the qualitative pattern of the perturbed QNM branches? Asymptotic analysis provides a sound approach. The study of the spiked TDP QNMs by Nollert and Price [45] provides an excellent illustration, with the identification of the large- $n$ asymptotic form of perturbed QNM branches, according to the logarithm dependence

$$
\operatorname{Im}\left(\omega_{n}\right) \sim C_{1}+C_{2} \ln \left(\operatorname{Re}\left(\omega_{n}\right)+C_{3}\right), \quad n \gg 1,
$$

with $C_{1}, C_{2}$, and $C_{3}$ being appropriate constants (note that $C_{3}$ can be put to zero for sufficiently high $n$, as in Ref. [45], since $\operatorname{Re}\left(\omega_{n}\right) \rightarrow \infty$ as $n \rightarrow \infty$; we prefer to keep it to account for intermediate asymptotics [57]). It is suggestive that this makes direct contact with the possible universality of perturbed $\mathrm{BH}$ QNMs and (nonperturbed) QNMs of compact objects evoked above. Indeed, as shown in Ref. [140], $w$ modes of (a class of) neutron stars present exactly this logarithm pattern [150]. Moreover, this makes (unexpected) contact with the Pöschl-Teller potential, where the spectral instability discussed in Sec. VB 2 is explained $[59,60,99,100]$ in terms of so-called broad Regge resonances (not to be confused with Regge poles), precisely described by such a logarithmic dependence [151] and explained in terms of the loss of continuity at a $p$ th order derivative, i.e., in terms of an underlying reduced $C^{p}$ regularity (with $p<\infty$ ). Along these lines of $C^{p}$ regularity, and in a WKB semiclassical analysis, such logarithmic branches have also been recovered in Ref. [47] in their recent discussion of Nollert's original work [44]. Therefore, it would be tempting to refer to the perturbed BH QNM branches as Nollert-Price-(Regge) QNMs, but this requires an elucidation of the role of the reduced $C^{p}$ regularity in the generic perturbations we have studied here, which, in particular, include $C^{\infty}$ regular (high-frequency) sinusoidal deterministic perturbations (63). In summary, the asymptotic pattern (76) provides a starting point to probe, in gravitational-wave signals, the physical properties (e.g., energy and frequency) of small-scale perturbations [57].

Beyond specific models, this kind of universal behavior, independent of the detailed nature of the high-frequency perturbation and valid for a large class of potentials, calls for systematic semiclassical analyses of highly damped scattering resonances, in terms of the wave-operator principal part [152], including boundary behaviors. In the spirit adopted in this work, we expect asymptotic tools in the semiclassical analysis of the pseudospectrum to provide a systematic approach to assess the universality of perturbed BH QNM branches [153].

\section{Overall perspective on Schwarzschild QNM instability}

The main result of this article is summarized in Fig. 17. Specifically, it combines Figs. 12-14 to demonstrate QNM spectral (in)stability through their three, respective, distinct calculations: (i) the calculation of the eigenfunctions of the exact spectral problem to calculate condition numbers $\kappa_{n}$, (ii) the evaluation of operator (matrix) norms to generate the pseudospectrum, and (iii) the calculation of eigenvalues of the perturbed spectral problem. Calculations (i) and (ii) work at the level of the unperturbed problem, whereas (iii) deals with the perturbed problem. The three calculations fit consistently through the Bauer-Fike theorem that constrains, through Eq. (39), the relation between the pseudospectrum and the tubular regions around the spectrum. They lead to these main results:

(i) QNM overtones

(i.1) QNM overtones are ultraviolet unstable, including the lowest overtones. The pseudospectrum provides a systematic explanatory and predictive framework for QNM spectral instability, confirming the result by Nollert and Price $[44,45]$. Such instability is indeed realized by physical high-frequency perturbations in the effective potential $V$, reaching the first overtone for sufficiently high frequencies and/or amplitudes in the perturbation.

(i.2) QNM overtones are stable under low-frequency perturbations. No instability appears for low- or intermediate-frequency perturbations of $V$, consistently with studies $[48-50,52,93]$ on astrophysical $\mathrm{BH}$ environments.

(ii) Slowest-decaying (fundamental) QNM

(ii.1) The slowest-decaying QNM is ultraviolet stable. This behavior holds, in Schwarzschild spacetime, for each $\ell$-fixed branch. This feature critically relies on keeping a faithful description of the asymptotic structure at infinity through the compactified hyperboloidal approach. This result is in contrast with conclusions in Refs. [44,45], but no contradiction appears since the latter implements a step-potential approximation fundamentally modifying $V$ at large distances, resulting, rather, in an infrared probe into QNMs.

(ii.2) The slowest-decaying QNM is stable under low-and intermediate-frequency perturbations in the potential. This property is shared by the whole QNM spectrum.

(ii.3) The slowest-decaying QNM is infrared unstable. The instability of the fundamental QNM observed in Refs. [44,45] is physical inasmuch as fundamental modifications of the large-distance structure of the potential are allowed.

(iii) Structural stability and QNM isospectrality

(iii.1) Nollert-Price BH QNM branches track pseudospectrum contour lines. The BH QNM spectrum is ultraviolet structurally unstable, migrating to perturbed branches tracking $\epsilon$-contour lines of pseudospectra. Such a migration pattern is largely independent of the detailed nature of high-frequency perturbations and potentials. Once on such NollertPrice branches, QNMs are spectrally stable. These 
structural stability properties result in the universality of perturbed QNM branches.

(iii.2) QNM isospectrality ultraviolet loss. High-frequency perturbations spoil the integrability of ReggeWheeler and Zerilli potentials, resulting (for perturbations reaching the first overtone) in a slightly enhanced damping of axial modes with respect to polar ones.

\section{CONCLUSIONS AND PERSPECTIVES}

\section{A. Conclusions}

We have demonstrated the following: (i) Fundamental BH QNMs are stable under high-frequency (ultraviolet) perturbations while unstable under (infrared) modifications of the asymptotics, the latter being consistent with Ref. [44]; (ii) (all) BH QNM overtones are unstable under high-frequency (ultraviolet) perturbations, instabilities being quantifiable in terms of the energy content (norm) of the perturbation, extending results in Refs. [44,45] to show isospectrality loss; and (iii) pseudospectrum contour lines provide the rationale underlying the structurally stable pattern of perturbed Nollert-Price BH QNM branches. Pseudospectra, together with tools from the analysis of non-self-adjoint operators, have revealed the analytic structure underlying such (in)stability properties of $\mathrm{BH}$ QNMs, offering an integrating and systematic approach to encompass a priori disparate phenomena. The soundness of the results relies on the use of a compactified hyperboloidal approach to QNMs, with the key identification of the relevant scalar product in the problem as associated with the physical energy, combined with accurate spectral numerical methods.

\section{Caveats in the current approach to QNM instability}

Beyond the soundness of the results, key questions remain:

(i) How much does the instability depend on the hyperboloidal approach? In other words, is the instability a property of the equation or rather of the employed scheme to cast it? This question is legitimate and crucial, requiring a specific investigation. In spite of this, we are confident in the soundness of our conclusions: As discussed in detail, the same qualitative behavior is found systematically by other studies not relying on the hyperboloidal approach, in particular, Nollert and Price's pioneer work. Details may change from scheme to scheme, but the (in) stability properties seem robust.

(ii) A numerical demonstration is not a proof. Moreover, numerical discretizations introduce their own difficulties and limitations. In particular, spectral issues in the passage from matrix approximations to the actual differential operator are a delicate problem. Again, we are confident in our results, as a consequence of mutual consistency of existing results and nontrivial tests like the ones described in the text. Proofs will definitely require the use of other methods and techniques.

(iii) Could the observed QNM spectral instability be an effect of regularity loss, namely, a $C^{p}$ effect? It may be, but it is difficult to conclude at this stage. Indeed, $C^{p}$ regularity provides a sufficient condition for logarithmic branches (76) that can be traced to works by Regge [151], Berry [155,156], or Zworski [100] and manifests in our setting in Nollert and Price's analysis of BH QNM instability [45] (complemented in Ref. [47]), in broad Regge resonances in Pöschl-Teller QNM instability [59,60,99], or in neutron star $w$ modes [140] (cf. also Ref. [148] dealing with related Regge poles). But we also attest to the same instability phenomenon for regular sinusoidal perturbations of sufficiently high frequency. Moreover, the pseudospectrum already informs us of the instability (cf. contour lines) at the unperturbed regular stage. If high frequency is actually the basic mechanism, then $C^{p}$ regularity would provide a sufficient, but not necessary condition for QNM instability. This point must be addressed.

\section{B. Perspectives}

While the pseudospectrum framework is already employed in physics (cf. e.g., Refs. [2,6,8,9,11]), there seems to be (to our knowledge) no systematic application in the gravitational context. The introduction of pseudospectra in gravitational physics opens an avenue to blend the study of instability and transients with other domains in physics (and beyond), by using pseudospectrum analysis as a common methodological frame. In the following, we mention some possible lines of exploration in different gravitational settings, from astrophysics and fundamental gravity physics to mathematical relativity, closing the discussion with a perspective beyond gravity.

\section{Astrophysics and cosmology}

The astrophysical status of the ultraviolet QNM overtone instability, which reaches the lowest overtones for generic perturbations of sufficiently high frequency and energy, requires us to assess whether actual astrophysical (and/or fundamental spacetime) perturbations are capable of triggering it. Some problems in which this question is relevant are the following:

(a) BH spectroscopy. If such instability is actually present, this should be taken into account in current approaches to $\mathrm{BH}$ spectroscopy. The stability of the slowestdecaying QNM guarantees that the dominating ringdown frequency is unaltered. But regarding QNM overtones, note that we have not referred at all to latetime ringdown frequencies, but to QNM frequencies: Since such sets of frequencies can actually decouple [44-47,53-56] and, as already noticed by Nollert [44], 
the propagating (scattered) field itself is not much affected by high-frequency perturbations, finding the signature of perturbed QNMs in the gravitationalwave signal may pose a very challenging problem [57]. Awareness of this potential effect in the GW signal may, however, lead to specifically tailored data analysis tools.

(b) BH environment. The arrangement of perturbed QNM branches along (a priori known) $\epsilon$-contour lines of pseudospectra opens the possibility of probing, in an inverse scattering spirit, environmental $\mathrm{BH}$ perturbations. One can envisage reading the size of the physical perturbations by comparing observational QNM data with the a priori calibrated pseudospectrum. This may help, for instance, to assess dry versus wet $\mathrm{BH}$ mergers, a point of cosmological relevance in LISA science.

(c) Universality of compact object QNMs. The combination of the universality of the perturbed Nollert-Price BH QNM branches with Nollert's remark on their similarity to neutron star $w$ modes, together with the demonstrated loss of BH QNM axial-polar isospectrality, poses a natural question: Do QNM spectra of all generic compact objects share the same pattern?

Schemes such as Ref. [23] may provide a systematic framework for the analysis of the astrophysical implications.

(d) BH QNM (in)stability in generic BHs. A natural and necessary extension of the present work is the study of QNM (in)stability in the full BH Kerr-Newman family, in particular, understanding how it intertwines with superradiance instability and the approach to extremality. Beyond asymptotic flatness, the study of QNM (in)stability should be extended to asymptotically de Sitter and Anti-De Sitter BHs.

\section{Fundamental gravitational physics}

We note some possible prospects at the fundamental level:

(a) (Sub)Planckian-scale physics. Planck scale spacetime fluctuations seem to be a robust prediction of different models of quantum gravity. They represent irreducible ultraviolet perturbations potentially providing a probe into Planck scale physics that, given the universality of BH QNM overtone instability, may be agnostic to an underlying theory of quantum gravity. Such a search of quantum gravity signatures in $\mathrm{BH}$ gravitationalwave physics is akin to Ref. [157]. Actually, it would suffice that a Planck scale cutoff induces an effective $C^{p}$ regularity in the otherwise smooth low-energy spacetime description, to trigger the instability phenomenon. BH QNM instability might then provide a particular probe into discreteness of spacetime (e.g., Ref. [158] are references therein).

(b) QNMs and (strong) cosmic censorship. In the setting of cosmological BHs, the assessment of the extendibility through the Cauchy horizon in Reissner-
Nordström-de Sitter spacetime is controlled by the parameter $\beta=\alpha / \kappa_{-}$, where $\alpha$ is the spectral gap (the imaginary part of the fundamental QNM in our setting) and $\kappa_{-}$is the surface gravity of the Cauchy horizon [29,159]. Therefore, a good understanding in this setting [cf. also point 1.(d) above] of the (in) stability properties of the slowest-decaying QNM, and, more generally, of the QNM spectrum, may be enlightening in the assessment of the thresholds for Cauchy horizon stability.

(c) Random perturbations and spacetime semiclassical limit. The regularization effect of random perturbations $[9,109-118]$ in the analytic structure of the scattering Green's function is an intriguing phenomenon that may play a role in the transition to a semiclassical, smooth, effective description of fundamental gravitational degrees of freedom described in a more basic (quantum) theory, possibly including an irreducible randomness ingredient. Again, the universality of the phenomenon may play a key role.

\section{Mathematical relativity}

The presented numerical evidence needs to be transformed into actual proofs. Some mathematical issues to address are as follows:

(a) Regularity conditions and QNM characterization. The mathematical study of QNMs entails subtle functional analysis issues. In the present hyperboloidal approach, this involves, in particular, the choice of appropriate regularity conditions and the associated functional space. This point connects our pseudospectrum study with the identification in Ref. [75] of the full uppercomplex plane as the actual QNM spectrum, if general $C^{\infty}$ eigenfunctions are allowed. More regularity must therefore be enforced. An analysis along the lines in Refs. [78-80], where Gevrey classes are identified as the proper functional spaces to define QNMs, is therefore required. Likewise, a systematic comparison with QNM stability in the framework of Refs. [29,77] is needed (cf. also Refs. [59,60]).

(b) Semiclassical analysis and QNM (in)stability. The interest of asymptotic tools, in the study of QNM stability, is twofold. On the one hand, an asymptotic reasoning [154] built on the semiclassical analysis of QNMs (a subject taken to full maturity in Sjöstrand's works [160-164]), with a small parameter defined in terms of highly damped QNM frequencies, can help us to assess universality patterns of perturbed NollertPrice BH QNM branches. On the other hand, asymptotic analysis provides powerful tools to rigorously prove spectral instability and nontrivial pseudospectra (cf. e.g., Ref. [165]). In particular, the recent work [166] provides an explicit example of scattering resonance (or QNM) instability, sharing much of the spirit of the discussion in this work. 


\section{Beyond gravitation: Gravity as a crossroad in physics}

The disclosure of BH QNM instability [44] resulted from the fluent interchange between gravitational and optical physics [48,102-104,167], again a key "flow channel" in our work, e.g., to understand the infrared instability of the fundamental QNM [108]. In this spirit, the present work can offer some hints for further boosting such kinds of transversal research in physics.

The hyperboloidal approach, with its explicit formulation of the dynamics in terms of a non-self-adjoint operator, provides a scheme of interest whenever dealing with an open physical system with losses at a radiation zone, a recurrent situation throughout physics (e.g., in optics, acoustics, and physical oceanography, to cite some settings). A specific lesson of the present work, to be exported to other physical contexts, is the identification of the relevant scalar product in terms of the system's energy, thus casting an a priori technical issue into neat physical terms. Moreover, when studying QNMs, the normalizability of the QNM eigenfunctions in the hyperboloidal approach may open an alternative avenue to the characterization of the so-called mode volume $V_{n}$ of a QNM, which is relevant, e.g., in the setting of photonic-plasmonic resonances [62]: Together with the notion of quality factor $Q_{n}$, given in terms of the ratio between the real and imaginary parts of a QNM (see, e.g., Ref. [168] for its connection with BH gravity physics), it characterizes the Purcell factor $F_{n} \sim Q_{n} / V_{n}$ controlling the enhancement of spontaneous emission of a quantum system, a key notion in cavity quantum electrodynamics [169].

Regarding the pseudospectrum, this notion is relevant whenever a non-Hermitian (or, more generally, non-selfadjoint operator) enters into the scene, as is typically the case in open systems [1]. In the context of non-Hermitian quantum mechanics, it has been proposed [8] to endow the pseudospectrum with a guiding central role in the theory, in a setting in which spectral instability makes insufficient the standard notion of the spectrum to fully characterize the relevant operators. Apart from spectral instability, the pseudospectrum underlies purely dynamical phenomena $[2,6]$, in particular, accounting for so-called nonmodal instability [170] in the setting of hydrodynamic stability theory and turbulence. Beyond hydrodynamics, the latter feature turns the pseudospectrum into a powerful tool for studying both spectral and dynamical stability issues in (open) physical systems that trace over a part of the total degrees of freedom and, as a result, are governed by non-self-ajoint operators. Such systems occur in many areas of physics (e.g., condensed matter, optics, plasmonics, acoustics, nanophysics, etc., [1]), offering a natural arena for extending the already large range of applications of pseudospectra [10].

Gravitational physics is remarkable in its capacity to "provide a framework that calls for the interchange of ideas, concepts and methodologies from very different communities" [171] in physics. The hyperboloidal approach and the pseudospectrum discussed here realize an instance of this understanding of "gravity as a crossroad in physics" [171].

\section{ACKNOWLEDGMENTS}

We thank M. Ansorg, P. Bizoń, O. Reula, and J. Sjöstrand for key insights. We also thank J. Olmedo, C. Barceló, L. Garay (and the rest of the Carramplas-2019 participants), L. Andersson, A. Ashtekar, E. Berti, N. Besset, I. Booth, Y. Boucher, V. Cardoso, G. Colas des Francs, M. Colbrook, A. Coutant, G. Cox, T. Daudé, K. Destounis, G. Dito, J. Frauendiener, H. Friedrich, D. Gajic, S. Guérin, D. Häfner, M. Hitrik, A. Iantchenko, H. R. Jauslin, J. Jezierski, B. Krishnan, J. Lampart, J. Lewandowski, M. Maliborski, M. Mokdad, J.-P. Nicolas, I. Racz, B. Raffaelli, A. Rostworowski, B. Sah, O. Sarbach, B. S. Sathyaprakash, J. Slipantschuk, A. Soumaila, J. A. Valiente-Kroon, and A. Zenginoglu. This work was supported by the French "Investissements d'Avenir" program through project ISITE-BFC (ANR-15-IDEX-03), the ANR "Quantum Fields interacting with Geometry" (QFG) project (ANR-20-CE40-0018-02), the EIPHI Graduate School (ANR-17-EURE-0002), the Spanish FIS201786497-C2-1 project (with FEDER contribution), the European Research Council Grant No. ERC-2014-StG 639022-NewNGR, "New frontiers in numerical general relativity," and the European Commission Marie Skłodowska-Curie Grant No. 843152 (Horizon 2020 programme). The project used Queen Mary's Apocrita HPC facility, supported by QMUL Research-IT, and CCuB computational resources (Université de Bourgogne).

\section{APPENDIX A: ENERGY SCALAR PRODUCT AND ADJOINT OPERATOR $L^{\dagger}$}

\section{Energy scalar product}

We start by considering the energy contained in the hyperboloidal slice $\Sigma_{\tau}$, defined by $\tau=$ const in Eq. (6), and associated with a mode $\phi_{\ell m}$ satisfying the effective equation (4), namely, propagation in $1+1$ Minkowski spacetime with a potential $V_{\ell}$ (see also Ref. [83]). In this stationary situation, this energy is given [67] by Eq. (18),

$$
E=\int_{\Sigma_{\tau}} T_{a b} t^{a} n^{b} d \Sigma_{\tau}
$$

The stress-energy tensor $T_{a b}=T_{a b}\left(\phi_{\ell m}, \nabla \phi_{\ell m}\right)$ of a (generally complex) scalar field is given by Eq. (17), with $\eta_{a b}$ the Minkowski metric in arbitrary coordinates [dropping $(\ell, m)]$,

$$
T_{a b}=\frac{1}{2}\left(\nabla_{a} \bar{\phi} \nabla_{b} \phi-\frac{1}{2} \eta_{a b}\left(\nabla^{c} \bar{\phi} \nabla_{c} \phi+V \bar{\phi} \phi\right)+\text { c.c. }\right),
$$

with "c.c." denoting "complex conjugate." Coming back to Eq. (A1), and using coordinates $(\tau, x)$ adapted to $\Sigma_{t}$ and defined in Eq. (6), the timelike Killing vector is $t^{a}=\partial_{t}=\frac{1}{\lambda} \partial_{\tau}$, and we have 


$$
\begin{aligned}
n^{a} & =\frac{1}{\sqrt{g^{\prime 2}-h^{\prime 2}}}\left(\frac{g^{\prime 2}-h^{\prime 2}}{\left|g^{\prime}\right|} \partial_{\tau}-\frac{h^{\prime}}{\left|g^{\prime}\right|} \partial_{x}\right) \\
& =\frac{1}{\sqrt{g^{\prime 2}-h^{\prime 2}}}\left(w(x) \partial_{\tau}-\gamma(x) \partial_{x}\right),
\end{aligned}
$$

for the timelike normal $n^{a}$, with $w(x)$ and $\gamma(x)$ defined in Eq. (12). Finally, the radial part of the metric integration measure $d \Sigma_{\tau}$ induced in the hyperboloidal slice $\Sigma_{\tau}$ (see details in Ref. [83] for the handling of the angular terms) is given by

$$
d \Sigma_{\tau}=\lambda \sqrt{g^{\prime 2}-h^{\prime 2}} d x .
$$

Inserting these elements in Eq. (A1), a straightforward calculation leads to Eq. (19), which we can rewrite as

$$
\begin{aligned}
E & =\frac{1}{2} \int_{a}^{b}\left(\frac{g^{\prime 2}-h^{\prime 2}}{\left|g^{\prime}\right|} \partial_{\tau} \bar{\phi} \partial_{\tau} \phi+\frac{1}{\left|g^{\prime}\right|} \partial_{x} \bar{\phi} \partial_{x} \phi+\left|g^{\prime}\right| \hat{V} \bar{\phi} \phi\right) d x \\
& =\frac{1}{2} \int_{a}^{b}\left(w(x) \partial_{\tau} \bar{\phi} \partial_{\tau} \phi+p(x) \partial_{x} \bar{\phi} \partial_{x} \phi+q(x) \bar{\phi} \phi\right) d x .
\end{aligned}
$$

Identifying $\psi=\partial_{\tau} \phi$, and taking $E$ for the square of the norm of the vector $u=(\phi, \psi)$, i.e., prescribing $\|u\|_{E}^{2}:=E$, we recover expression (20). Considering only the $\phi$ part, this "energy norm" is an $H^{1}$-like norm, so it takes into account the frequency (actually the wave number) of the mode $\phi$, a most important ingredient in our setting, given the role of high-frequency perturbations in the ultraviolet instability of QNM overtones. Finally, considering the whole $u=(\phi, \psi)$ vector, this norm is an $L^{2}$ norm coming from the energy scalar product $\langle\cdot, \cdot\rangle_{E}$ [for $q(x)>0$ ],

$$
\begin{aligned}
& \left\langle\left(\begin{array}{l}
\phi_{1} \\
\psi_{1}
\end{array}\right),\left(\begin{array}{l}
\phi_{2} \\
\psi_{2}
\end{array}\right)\right\rangle_{E} \\
& =\frac{1}{2} \int_{a}^{b}\left(w(x) \bar{\psi}_{1} \psi_{2}+p(x) \partial_{x} \bar{\phi}_{1} \partial_{x} \phi_{2}+q(x) \bar{\phi}_{1} \phi_{2}\right) d x,
\end{aligned}
$$

which coincides with Eq. (22) upon identification, $q(x)=\tilde{V}$. Note that $\gamma(x)$ plays no role in the energy scalar product $\langle\cdot, \cdot\rangle_{E}$.

\section{Adjoint operator $\boldsymbol{L}^{\dagger}$}

A very important object in our discussion of QNM spectral instability and the pseudospectrum construction is the adjoint $L^{\dagger}$ of the operator $L$. The definition of $L^{\dagger}$ depends on the choice of scalar product, and we shall adopt here the energy scalar product (A7). The full construction of the adjoint $L^{\dagger}$ requires a discussion of its domain of dependence. This is a delicate question, which is intimately linked with the boundary and regularity conditions determining the functional space on which $L$ and $L^{\dagger}$ are defined. This functional analysis issue will be addressed elsewhere, and here we focus on the construction of the so-called formal adjoint, formally satisfying the relation

$$
\left\langle L^{\dagger}\left(\begin{array}{l}
\phi_{1} \\
\psi_{1}
\end{array}\right),\left(\begin{array}{l}
\phi_{2} \\
\psi_{2}
\end{array}\right)\right\rangle_{E}=\left\langle\left(\begin{array}{l}
\phi_{1} \\
\psi_{1}
\end{array}\right), L\left(\begin{array}{l}
\phi_{2} \\
\psi_{2}
\end{array}\right)\right\rangle_{E},
$$

for all $u_{1}=\left(\phi_{1}, \psi_{1}\right)$ and $u_{2}=\left(\phi_{2}, \psi_{2}\right)$. Taking into account the definition in Eq. (10) of the operator $L$, we write

$$
\begin{aligned}
\left\langle L^{\dagger}\left(\begin{array}{l}
\phi_{1} \\
\psi_{1}
\end{array}\right),\left(\begin{array}{c}
\phi_{2} \\
\psi_{2}
\end{array}\right)\right\rangle_{E} & =\left\langle\left(\begin{array}{l}
\phi_{1} \\
\psi_{1}
\end{array}\right), \frac{1}{i}\left(\begin{array}{cc}
0 & 1 \\
\hline L_{1} & L_{2}
\end{array}\right)\left(\begin{array}{c}
\phi_{2} \\
\psi_{2}
\end{array}\right)\right\rangle_{E} \\
& =\left\langle\left(\begin{array}{c}
\phi_{1} \\
\psi_{1}
\end{array}\right), \frac{1}{i}\left(\begin{array}{c}
\psi_{2} \\
L_{1} \phi_{2}+L_{2} \psi_{2}
\end{array}\right)\right\rangle_{E} \\
& =\left\langle\left(\begin{array}{l}
\phi_{1} \\
\psi_{1}
\end{array}\right), \frac{1}{i}\left(\frac{1}{w(x)}\left(\partial_{x}\left(p(x) \partial_{x} \phi_{2}\right)-q(x) \phi_{2}+2 \gamma(x) \partial_{x} \psi_{2}+\partial_{x} \gamma(x) \psi_{2}\right)\right)\right\rangle_{E},
\end{aligned}
$$

where we have used the expressions for $L_{1}$ and $L_{2}$ in Eq. (11). Using the energy scalar product (A7) and integrating by parts,

$$
\begin{aligned}
& \left\langle L^{\dagger}\left(\begin{array}{c}
\phi_{1} \\
\psi_{1}
\end{array}\right),\left(\begin{array}{c}
\phi_{2} \\
\psi_{2}
\end{array}\right)\right\rangle_{E}=\left\langle\frac{1}{i}\left(\begin{array}{c}
\psi_{1} \\
\frac{1}{w(x)}\left(\partial_{x}\left(p(x) \partial_{x} \phi_{1}\right)-q(x) \phi_{1}+2 \gamma(x) \partial_{x} \psi_{1}+\partial_{x} \gamma(x) \psi_{1}\right)
\end{array}\right),\left(\begin{array}{c}
\phi_{2} \\
\psi_{2}
\end{array}\right)\right\rangle_{E} \\
& +\frac{1}{i}\left(2 \gamma(b) \bar{\psi}_{1}(b) \psi_{2}(b)-2 \gamma(a) \bar{\psi}_{1}(a) \psi_{2}(a)\right) \\
& =\left\langle\frac{1}{i}\left(\begin{array}{c|c}
0 & 1 \\
\hline L_{1} & L_{2}
\end{array}\right)\left(\begin{array}{l}
\phi_{1} \\
\psi_{1}
\end{array}\right),\left(\begin{array}{l}
\phi_{2} \\
\psi_{2}
\end{array}\right)\right\rangle_{E}+\int_{a}^{b} w(x)\left(\overline{\frac{1}{i} 2 \frac{\gamma(x)}{w(x)}(\delta(x-a)-\delta(x-b)) \psi_{1}}\right) \psi_{2} d x,
\end{aligned}
$$

where we have used $p(a)=p(b)=0$, the real character of $w(x), p(x), q(x)$, and $\gamma(x)$, and the Dirac-delta $\delta(x)$ distribution to formally evaluate the boundary terms. Thus, we can rewrite 


$$
\left\langle L^{\dagger}\left(\begin{array}{c}
\phi_{1} \\
\psi_{1}
\end{array}\right),\left(\begin{array}{c}
\phi_{2} \\
\psi_{2}
\end{array}\right)\right\rangle_{E}=\left\langle\frac{1}{i}\left(\begin{array}{c|c}
0 & 1 \\
\hline L_{1} & L_{2}+2 \frac{\gamma(x)}{w(x)}(\delta(x-a)-\delta(x-b))
\end{array}\right)\left(\begin{array}{c}
\phi_{1} \\
\psi_{1}
\end{array}\right),\left(\begin{array}{c}
\phi_{2} \\
\psi_{2}
\end{array}\right)\right\rangle_{E}
$$

so that, introducing the operator $L_{2}^{\partial}$ as in Eq. (25),

$$
L_{2}^{\partial}=2 \frac{\gamma(x)}{w(x)}(\delta(x-a)-\delta(x-b)),
$$

we can write the formal adjoint in Eqs. (23) and (24),

$$
L^{\dagger}=L+L^{\partial}, \quad L^{\partial}=\frac{1}{i}\left(\begin{array}{c|c}
0 & 0 \\
\hline 0 & L_{2}^{\partial}
\end{array}\right) .
$$

In general, $\gamma(x)$ does not vanish at the boundaries, so $L$ is not even symmetric and therefore cannot be self-adjoint. Equation (A13) neatly identifies the loss of self-adjointness with such nonvanishing $\gamma(x)$, specifically linking spectral instability with a boundary phenomenon, formally cast through the presence of the Dirac-delta terms. This form also explains the (formal) self-adjoint case $L_{2}=0$ discussed in Sec. V C 2.

More generally, evaluation of adjoints plays a key role in all aspects of our discussion of spectral instability: (i) calculation of conditions numbers $\kappa_{n}$, involving the spectral problem of the adjoint $L^{\dagger}$, cf. Eq. (26); (ii) evaluation of the pseudospectrum, involving the calculation of (generalized) singular values of $R_{L}(\omega)$ and therefore the spectral problem of $R_{L}^{\dagger}(\omega) R_{L}(\omega)$, cf. Eqs. (43) and (B21); and (iii) the prescription of the norm $\|\delta L\|_{E}$ (that we have systematically denoted $\|\delta \tilde{V}\|_{E}$ ) to $\epsilon$ in the exploration of perturbed spectral QNM problems, again involving the spectral problem of the operator $\delta L^{\dagger} \delta L$. Details of the calculation of adjoints in our discretized approach are given in Appendixes B and C.

\section{APPENDIX B: PSEUDOSPECTRUM IN THE ENERGY NORM}

Here, we derive the relevant expressions for the construction of pseudospectra in the discretized version of the energy norm.

\section{Scalar product and adjoint}

Let us consider a general Hermitian-scalar product in $\mathbb{C}^{n}$ as

$$
\langle u, v\rangle_{G}=\left(u^{*}\right)^{i} G_{i j} v^{j}=u^{*} \cdot G \cdot v,
$$

with $G$ a positive-definite Hermitian matrix,

$$
G^{*}=G, \quad x^{*} \cdot G \cdot x>0 \quad \text { if } x \neq 0,
$$

where ${ }^{*}$ denotes conjugate-transpose, i.e., $u^{*}=\bar{u}^{t}$ and $G^{*}=\bar{G}^{t}$ (we notice that, in the problem studied in this work, the Hermitian positive-definite matrix $G$ is actually a real symmetric positive-definite matrix $G^{t}=G$, but we keep the discussion in full generality). Using Eqs. (B1) and (B2) in the relation

$$
\left\langle A^{\dagger} u, v\right\rangle_{G}=\langle u, A v\rangle_{G},
$$

characterizing the adjoint $A^{\dagger}$ of $A$ with respect to the scalar product (B1), we immediately get

$$
A^{\dagger}=G^{-1} A^{*} G
$$

\section{Induced matrix norm from a scalar product norm}

The (vector) norm $\|\cdot\|_{G}$ in $\mathbb{C}^{n}$ associated with the scalar product $\langle\cdot, \cdot\rangle_{G}$ in Eq. (B1), namely,

$$
\|v\|_{G}=\left(\langle v, v\rangle_{G}\right)^{\frac{1}{2}}
$$

induces a matrix norm $\|\cdot\|_{G}$ in $M_{n}(\mathbb{C})$ defined as

$$
\|A\|_{G}=\max _{\|x\|_{G}=1, x \in \mathbb{C}^{n}}\left\{\|A x\|_{G}\right\}, A \in M_{n}(\mathbb{C}) .
$$

A more useful characterization of this $L^{2}$ induced matrix norm is given in terms of the spectral radius $\rho\left(A^{\dagger} A\right)$ of $A^{\dagger} A$, where

$$
\rho(M)=\max _{\lambda \in \sigma(M)}\{|\lambda|\}
$$

Indeed, we can write

$$
\begin{aligned}
\|A\|_{G}^{2} & =\left(\max _{\|x\|_{G}=1, x \in \mathbb{C}^{n}}\left\{\left(\langle A x, A x\rangle_{G}\right)^{\frac{1}{2}}\right\}\right)^{2} \\
& =\max _{\|x\|_{G}=1, x \in \mathbb{C}^{n}}\left\{\langle A x, A x\rangle_{G}\right\} \\
& =\max _{\|x\|_{G}=1, x \in \mathbb{C}^{n}}\left\{\left\langle A^{\dagger} A x, x\right\rangle_{G}\right\} .
\end{aligned}
$$

The rest of the argument essentially follows from the Rayleigh-Ritz formula for self-adjoint operators. Explicitly, the (self-adjoint) matrix $A^{\dagger} A$ is unitarily diagonalizable and non-negative definite (that is, $\left.\left\langle x, A^{\dagger} A x\right\rangle_{G} \geq 0, \forall x \in \mathbb{C}^{n}\right)$, so we can find an orthonormal basis of eigenvectors $\left\{e_{i}\right\}$, 


$$
A^{\dagger} A e_{i}=\lambda_{i} e_{i}, \quad\left\langle e_{i}, e_{j}\right\rangle_{G}=\delta_{i j},
$$

with real non-negative eigenvalues $\lambda_{i}$ that we order as

$$
0 \leq \lambda_{1} \leq \lambda_{2} \ldots \leq \lambda_{n} .
$$

Expanding $x=\sum_{i} x^{i} e_{i}$ for an arbitrary $x \in \mathbb{C}^{n}$, we write

$$
\left\langle A^{\dagger} A x, x\right\rangle_{G}=\sum_{i} \lambda_{i}\left|x^{i}\right|^{2} \leq \lambda_{n} \sum_{i}\left|x^{i}\right|^{2}=\lambda_{n}\|x\|_{G}^{2},
$$

which we can recast as

$$
\left\langle A^{\dagger} A \frac{x}{\|x\|_{G}}, \frac{x}{\|x\|_{G}}\right\rangle_{G} \leq \lambda_{n}=\rho\left(A^{\dagger} A\right) .
$$

Inserting this into Eq. (B8), we conclude

$$
\|A\|_{G}^{2} \leq \rho\left(A^{\dagger} A\right) \text {. }
$$

To prove that the inequality is actually saturated, it suffices to show that there exists a vector $x,\|x\|_{G}=1$, that realizes the equality, i.e., $\|A x, A x\|_{G}^{2}=\rho\left(A^{\dagger} A\right)$. If we consider $x=e_{n}$,

$$
\begin{aligned}
\left\|A e_{n}\right\|_{G}^{2} & =\left\langle A e_{n}, A e_{n}\right\rangle_{G}=\left\langle A^{\dagger} A e_{n}, e_{n}\right\rangle_{G} \\
& =\lambda_{n}=\rho\left(A^{\dagger} A\right),
\end{aligned}
$$

and we can finally conclude

$$
\|A\|_{G}=\left(\rho\left(A^{\dagger} A\right)\right)^{\frac{1}{2}} .
$$

\section{Characterization of the pseudospectrum}

Given an invertible matrix $A \in M_{n}(\mathbb{C})$ and a nonvanishing eigenvalue $\lambda$, then $1 / \lambda$ is an eigenvalue of $A^{-1}$ and

$$
\max _{\lambda \in \sigma\left(A^{-1}\right)}\{|\lambda|\}=\left(\min _{\lambda \in \sigma(A)}\{|\lambda|\}\right)^{-1}
$$

Then, for an invertible $M \in M_{n}(\mathbb{C})$, we can write for the squared norm $\|\cdot\|_{G}$ of its inverse $M^{-1}$,

$$
\begin{aligned}
\left\|M^{-1}\right\|_{G}^{2} & =\rho\left(\left(M^{-1}\right)^{\dagger} M^{-1}\right)=\rho\left(\left(M M^{\dagger}\right)^{-1}\right) \\
& =\left(\min _{\lambda \in \sigma\left(M M^{\dagger}\right)}\{\lambda\}\right)^{-1} \\
& =\left(\min _{\lambda \in \sigma\left(M^{\dagger} M\right)}\{\lambda\}\right)^{-1}
\end{aligned}
$$

where in the passage from the first line to the second, we have used Eq. (B16) and the definition (B7) of the spectral radius, whereas in the last equality, we have used that a matrix $A B$ has the same eigenvalues as a matrix $B A$.

We now consider the $\epsilon$-pseudospectrum characterization in Definition 2, namely, Eq. (32), applied to the discretized energy norm $\|\cdot\|_{G}$,

$$
\sigma_{G}^{\epsilon}(A)=\left\{\lambda \in \mathbb{C}:\left\|(\lambda \mathrm{Id}-A)^{-1}\right\|_{G}>1 / \epsilon\right\} .
$$

Using Eq. (B17), with $M=\lambda \mathrm{Id}-A$, we can write

$$
\left\|(\lambda \mathrm{Id}-A)^{-1}\right\|_{G}>1 / \epsilon \Leftrightarrow \epsilon>\left(\min _{\lambda \in \sigma\left(M^{\dagger} M\right)}\{\lambda\}\right)^{\frac{1}{2}} .
$$

Finally, $\sigma_{G}^{\epsilon}(A)$ can be written as

$$
\sigma_{G}^{\epsilon}(A)=\left\{\lambda \in \mathbb{C}: s_{G}^{\min }(\lambda \mathrm{Id}-A)<\epsilon\right\},
$$

where $s_{G}^{\min }(M)$ is the minimum of a set of generalized singular values of $M$, related to the $\langle\cdot, \cdot\rangle_{G}$ scalar product

$$
s_{G}^{\min }(M):=\min \left\{\sqrt{\lambda}: \lambda \in \sigma\left(M^{\dagger} M\right)\right\} .
$$

When choosing the energy scalar product in Sec. IV, that is, with $G=G^{E}$ (see explicit expression in Appendix C), we recover expression (43) for $\sigma_{E}^{\epsilon}(A)$. When using the canonical $L^{2}$ product, we recover the standard $\sigma_{2}^{\epsilon}(A)$ in Eq. (40), where

$$
s_{2}^{\min }(M)=\min \left\{\sqrt{\lambda}: \lambda \in \sigma\left(M^{*} M\right)\right\}=: \sigma^{\min }
$$

is the smallest of the singular values $\sigma_{i}(M)=\sqrt{\lambda_{i}}$, $\lambda_{i} \in \sigma\left(M^{*} M\right)$, in the standard singular value decomposition of $M$.

\section{APPENDIX C: ELEMENTS IN THE CHEBYSHEV DISCRETIZATION}

\section{Chebyshev spectral decomposition}

The Chebyshev's polynomial of order $k$ is given by

$$
T_{k}(x)=\cos (k \arccos x), \quad x \in[-1,1] .
$$

Chebyshev's polynomials provide an orthogonal basis for functions $f \in L^{2}([-1,1], w(x) d x)$, with $w(x)=1 / \sqrt{1-x^{2}}$, so we can write the spectral expansion

$$
f(x)=\frac{c_{0}}{2}+\sum_{k=1}^{\infty} c_{k} T_{k}(x) .
$$

For sufficiently regular functions $f(x)$, coefficients $c_{k}$ decay exponentially in $k$. An $f_{N}(x)$ approximate of $f(x)$ is obtained by truncating the series to order $N$,

$$
f_{N}(x)=\frac{c_{0}}{2}+\sum_{k=1}^{N} c_{k} T_{k}(x) .
$$

The function $f$ is therefore approximated by the vector $\left(c_{0}, c_{1}, \ldots, c_{N}\right)$ in $\mathbb{C}^{n}$, with $n=N+1$. In particular, we can evaluate the integral of $f$ in the interval $[-1,1]$ as 


$$
\int_{-1}^{1} f_{N}(x) d x=c_{0}-\sum_{k=1}^{\left\lfloor\frac{N}{2}\right\rfloor} \frac{c_{2 k}}{4 k^{2}-1}
$$

\section{Collocation methods: Chebyshev-Lobatto grid}

When dealing with the product of functions, as is the case in our setting, the description in terms of spectral coefficients $c_{i}$ is not convenient. Instead, one constructs a Chebyshev's interpolant $f_{N}(x)$ from the evaluation of $f(x)$ on points $x_{i}$,

$$
f_{N}\left(x_{i}\right)=f\left(x_{i}\right), \quad i \in\{0,1, \ldots, N\},
$$

where $x_{i} \in[-1,1]$ define an appropriately chosen $n$-point quadrature grid. For concreteness, in the following, we focus on the Chebyshev-Lobatto collocation grid including the interval boundaries $x= \pm 1$, in the spirit of including horizon and null infinity points in our compactified picture. The Chebyshev-Lobatto $(N+1)$ grid is given by the extrema of $T_{N}(x)$ [i.e., the $N-1$ zeros of $T_{N}^{\prime}(x)$ ] together with both extreme points $x_{0}=1$ and $x_{N}=-1$, resulting in the values

$$
x_{i}=\cos \left(\frac{\pi i}{N}\right), \quad i \in\{0,1, \ldots, N\} .
$$

We can enforce Eq. (C5) on this grid by constructing an $f_{N}(x)$ interpolant in the functional form (C3), with coefficients [172]

$c_{i}=\frac{2-\delta_{i N}}{2 N}\left[f\left(x_{0}\right)+(-1)^{i} f\left(x_{N}\right)+2 \sum_{j=1}^{N-1} f\left(x_{j}\right) T_{i}\left(x_{j}\right)\right]$,

with $i \in\{0,1, \ldots, N\}$. In the construction of our differential operator $L$, the interpolant of the product of two functions $f$ and $g$ is obtained by multiplication on grid points, that is,

$$
(f g)_{N}\left(x_{i}\right)=f_{N}\left(x_{i}\right) g_{N}\left(x_{i}\right) \text {. }
$$

In addition, we need an expression for the interpolant of the derivative $f_{N}^{\prime}(x)=(d f / d x)_{N}(x)$, which is determined by

$$
f_{N}^{\prime}\left(x_{i}\right)=\sum_{j=0}^{N} \mathbb{D}_{i j}^{N} f_{N}\left(x_{j}\right)
$$

with

$$
\mathbb{D}_{i j}^{N}=\left\{\begin{array}{lc}
-\frac{2 N^{2}+1}{6} & i=j=N \\
\frac{2 N^{2}+1}{6} & i=j=0 \\
-\frac{x_{j}}{2\left(1-x_{j}\right)^{2}} & 0<i=j<N \\
\frac{\alpha_{i}}{\alpha_{j}} \frac{(-1)^{i-j}}{x_{i}-x_{j}} & i \neq j,
\end{array}\right.
$$

where

$$
\alpha_{i}= \begin{cases}2 & i \in\{0, N\} \\ 1 & i \in\{1, \ldots, N-1\} .\end{cases}
$$

\section{Energy scalar product: Gram matrix $G^{E}$}

Let us first consider the integral

$$
I_{\mu}(f, g)=\int_{-1}^{1} f(x) g(x) d \mu,
$$

with $d \mu=\mu(x) d x$. We can get a quadrature approximation $I_{\mu}^{N}(f, g)$ to $I_{\mu}(f, g)$ by using expression (C4) for $N$ interpolants $f_{N}$ and $g_{N}$ in Eq. (C3) combined with the particular expression (C7) for coefficients in the Chebyshev-Lobatto grid and the grid multiplication in Eq. (C8). We then obtain

$$
I_{\mu}^{N}(f, g)=f_{N}^{t} \cdot C_{\mu}^{N} \cdot g_{N},
$$

with $f_{N}^{t}=\left(f\left(x_{0}\right), \ldots, f\left(x_{N}\right)\right), g_{N}^{t}=\left(g\left(x_{0}\right), \ldots, g\left(x_{N}\right)\right)$ the $(N+1)$-grid approximates of $f$ and $g$, respectively, and $C_{\mu}^{N}$ the diagonal matrix given by

$$
\begin{aligned}
\left(C_{\mu}^{N}\right)_{i j} & =\left(C_{\mu}^{N}\right)_{i} \delta_{i j}, \\
\left(C_{\mu}^{N}\right)_{i} & =\frac{2 \mu\left(x_{i}\right)}{\alpha_{i} N}\left(1-\sum_{k=1}^{\left\lfloor\frac{N}{2}\right\rfloor} T_{2 k}\left(x_{i}\right) \frac{2-\delta_{2 k, N}}{4 k^{2}-1}\right),
\end{aligned}
$$

where we have used $T_{0}(x)=1, T_{k}(1)=1$, and $T_{k}(-1)=$ $(-1)^{k}$. Then, dropping the indices $N$, we can write the discrete version of the scalar product $\langle\cdot, \cdot\rangle_{E}$ in Eq. (22) as

$$
\begin{aligned}
\left\langle u_{1}, u_{2}\right\rangle_{E}= & \left\langle\left(\begin{array}{c}
\phi_{1} \\
\psi_{1}
\end{array}\right),\left(\begin{array}{c}
\phi_{2} \\
\psi_{2}
\end{array}\right)\right\rangle_{E} \\
= & \frac{1}{2}\left(\psi_{1}^{*} \cdot C_{w} \cdot \psi_{2}+\left(\mathbb{D} \phi_{1}\right)^{*} \cdot C_{p} \cdot \mathbb{D} \phi_{1}\right. \\
& \left.+\phi_{1}^{*} \cdot C_{\tilde{V}_{e}} \cdot \phi_{2}\right),
\end{aligned}
$$

which can be rewritten in matrix form as

$$
\begin{aligned}
\left\langle u_{1}, u_{2}\right\rangle_{E} & =u_{1}^{*} \cdot G^{E} \cdot u_{2} \\
& =\left(\bar{\phi}_{1}, \bar{\psi}_{1}\right)\left(\begin{array}{c|c}
G_{1}^{E} & 0 \\
\hline 0 & G_{2}^{E}
\end{array}\right)\left(\begin{array}{c}
\phi_{2} \\
\psi_{2}
\end{array}\right),
\end{aligned}
$$


with [here, the matrices $C_{\tilde{V}_{e}}, C_{p}$, and $C_{w}$ are given by Eq. (C14) for the respective functions $\mu(x)=$ $\left.\tilde{V}_{\ell}(x), p(x), w(x)\right]$

$$
\begin{aligned}
G_{1}^{E} & =\frac{1}{2}\left(C_{\tilde{V}_{\ell}}+\mathbb{D}^{t} \cdot C_{p} \cdot \mathbb{D}\right), \\
G_{2}^{E} & =\frac{1}{2} C_{w} .
\end{aligned}
$$

These expressions define the Gram matrix $G^{E}$ for the discretized version of the energy scalar product (22), in the basis determined from the Chebyshev-Lobatto spectral grid.

\section{a. Grid interpolation}

An important aspect to observe when performing the numerical integration is that Eq. (C4) is exact whenever the original function $f(x)$ is a polynomial of order less than or equal to $N$. With this in mind, and assuming that $f(x)$ and $g(x)$ are polynomials, Eq. (C13) is exact only for the case where the product $(f g)(x)$ yields polynomials of order less than or equal to $N$. In practical terms, the procedure described above hampers the accuracy of the scalar product's numerical integration whenever the order becomes greater than $N$.

As an illustrative example, take $f(x)=P_{\ell}(x)$ and $g(x)=P_{\ell^{\prime}}(x)$, with $P_{\ell}(x)$ the Legendre polynomials. Then, the integral (C12) - with $\mu(x)=1$ omitted from the expression-yields $I(f, g)=2 \delta_{\ell, \ell^{\prime}} /(2 \ell+1)$. If we now consider the discrete version $I^{N}(f, g)$ given by Eq. (C13), one observes that the exact result is obtained only for the cases $\ell+\ell^{\prime} \leq N$, even though each individual function $f(x)$ or $g(x)$ is exactly represented for $\ell \leq N$ and $\ell^{\prime} \leq N$, respectively.

To mitigate this issue, we modify the integration matrix $C_{\mu}^{N}$-or, equivalently, the Gram matrix $G^{E}$-by incorporating the following interpolation strategy.

Given an interpolant vector $f_{N}\left(x_{i}\right)$ associated with a Chebyshev-Lobatto grid $\left\{x_{i}\right\}_{i=0}^{N}$, one can obtain a second interpolant vector $f_{\bar{N}}\left(\bar{x}_{i}\right)$ associated with another Chebyshev-Lobatto grid $\left\{\bar{x}_{i}\right\}_{i=0}^{\bar{N}}$ with a resolution $N \neq \bar{N}$ via

$$
f_{\bar{N}}\left(\bar{x}_{i}\right)=\sum_{i=0}^{N} \mathbb{I}_{\bar{i} i} f_{N}\left(x_{i}\right)
$$

Components $\mathbb{I}_{\bar{i} i}$ of the interpolation matrix $\mathbb{I}$ are obtained by evaluating Eq. (C3) at the grid $\left\{\bar{x}_{i}\right\}_{i=0}^{\bar{N}}$, with the coefficients $\left\{c_{i}\right\}_{i=0}^{N}$ expressed in terms of $f_{N}\left(x_{i}\right)$ via Eq. (C7). Then,

$$
\mathbb{I}_{\overline{i i}}=\frac{1}{\alpha_{i} N}\left(1+\sum_{j=1}^{N}\left(2-\delta_{j, N}\right) T_{j}\left(\bar{x}_{i}\right) T_{j}\left(x_{i}\right)\right) .
$$

Note that the interpolation matrix $\mathbb{I}$ has size $\bar{N} \times N$, which reduces to a square matrix only if $\bar{N}=N$. In this case, Eq. (C19) is actually the identity matrix, as expected.

Then, for a fixed $N$, we consider the discrete integration (C13) in terms of a higher resolution $\bar{N}=2 N$ and interpolate the expression back to the original resolution $N$. In other words, defining $\mathcal{I}_{\mu}^{N}(f, g):=I_{\mu}^{\bar{N}}(f, g)$, we can consider the new, grid-interpolated, discrete integration

$$
\mathcal{I}_{\mu}^{N}(f, g)=f_{N}^{t} \cdot \mathcal{C}_{\mu}^{N} \cdot g_{N}
$$

where $\mathcal{C}_{\mu}^{N}=\mathbb{I}^{t} \cdot C_{\mu}^{\bar{N}} \cdot \mathbb{I}$ or, in terms of its components

$$
\left(\mathcal{C}_{\mu}^{N}\right)_{i j}=\sum_{\bar{i}=0}^{\bar{N}} \sum_{\bar{j}=0}^{\bar{N}}\left(\mathbb{I}^{t}\right)_{i \bar{i}}\left(C_{\mu}^{\bar{N}}\right)_{\bar{i}} \overline{\mathbb{j}}_{\bar{j} j}
$$

Going back to the illustrative example where $f(x)=$ $P_{\ell}(x)$ and $g(x)=P_{\ell^{\prime}}(x)$, we now obtain $\mathcal{I}^{N}(f, g)=$ $2 \delta_{\ell, \ell^{\prime}} /(2 \ell+1)$ exactly whenever $\ell, \ell^{\prime} \leq N$.

In the same way, we grid interpolate the Gram matrices

$$
\mathcal{G}_{1}^{E}=\mathbb{I}^{t} \cdot G_{1}^{E} \cdot \mathbb{I}, \quad \mathcal{G}_{2}^{E}=\mathbb{I}^{t} \cdot G_{2}^{E} \cdot \mathbb{I},
$$

which allows us to perform the scalar product $(\mathrm{C} 16)$ via

$$
\begin{aligned}
\left\langle u_{1}, u_{2}\right\rangle_{E} & =u_{1}^{*} \cdot \mathcal{G}^{E} \cdot u_{2} \\
& =\left(\bar{\phi}_{1}, \bar{\psi}_{1}\right)\left(\begin{array}{c|c}
\mathcal{G}_{1}^{E} & 0 \\
\hline 0 & \mathcal{G}_{2}^{E}
\end{array}\right)\left(\begin{array}{c}
\phi_{2} \\
\psi_{2}
\end{array}\right) .
\end{aligned}
$$

\section{APPENDIX D: PÖSCHL-TELLER QNMS AND REGULARITY}

Here, we give the derivation of Pöschl-Teller QNM frequencies (and QNM eigenfunctions in our setting), which we do for completeness and, more importantly, to illustrate, with an explicit example, the role of regularity in the enforcement of outgoing boundary conditions in the hyperboloidal scheme.

We start from the Fourier transform in time of the PöschlTeller wave equation in Bizoń-Mach coordinates, i.e., Eq. (56),

$$
\left(\left(1-x^{2}\right) \frac{d^{2}}{d x^{2}}-2(i \omega+1) x \frac{d}{d x}-i \omega(i \omega+1)-1\right) \phi=0 .
$$

This equation can be solved in terms of hypergeometric functions. Making the change $x=1-2 z$, it is rewritten as 


$$
\begin{gathered}
\left(z(1-z) \frac{d^{2}}{d z^{2}}+((1+i \omega)-2(1+i \omega) z) \frac{d}{d z}\right. \\
-(i \omega(i \omega+1)+1)) \phi=0
\end{gathered}
$$

namely, Euler's hypergeometric differential equation

$$
\left(z(1-z) \frac{d^{2}}{d z^{2}}+(c-(a+b+1) z) \frac{d}{d z}-a b\right) \phi=0,
$$

for the values

$$
\begin{aligned}
& c=1+i \omega, \\
& a=\frac{(2 i \omega+1) \pm i \sqrt{3}}{2}, \\
& b=(2 i \omega+1)-a=\frac{(2 i \omega+1) \mp i \sqrt{3}}{2} .
\end{aligned}
$$

For each choice of $\omega$, this equation admits two linearly independent solutions that can be built from the Gauss hypergeometric function ${ }_{2} F_{1}(a, b ; c ; z)$. It is only when we enforce some regularity in the solution that the spectral parameter $\omega$ is discretized and we recover the QNM frequencies. In this particular case, it is when we truncate the hypergeometric series ${ }_{2} F_{1}(a, b ; c ; z)$ to a polynomial that we recover Pöschl-Teller QNM frequencies. Such truncation occurs when either $a$ or $b$ is a nonpositive integer. From Eq. (D4), we can write

$$
\omega=\mp \frac{\sqrt{3}}{2}+i\left(-a+\frac{1}{2}\right)= \pm \frac{\sqrt{3}}{2}+i\left(-b+\frac{1}{2}\right) .
$$

Therefore, imposing either $a=-n$ or $b=-n$, with $n \in \mathbb{N} \cup\{0\}$, we finally get

$$
\omega_{n}^{ \pm}= \pm \frac{\sqrt{3}}{2}+i\left(n+\frac{1}{2}\right)
$$

Choosing the $a=-n$ version, the corresponding eigenvectors can be written as Jacobi polynomials $P_{n}^{(\alpha, \beta)}(x)$, defined as

$$
P_{n}^{(\alpha, \beta)}(x)=\frac{(\alpha+1)}{n !}{ }_{2} F_{1}\left(-n, 1+\alpha+\beta ; \alpha+1 ; \frac{1-x}{2}\right),
$$

with $(y)_{n}$ the Pochhammer symbol [i.e., $(y)_{n}=\prod_{k=0}^{n-1}(y-k)$ ]. Inserting, for a given $n \in \mathbb{N} \cup\{0\}$, the values in Eqs. (D4) and (D6) into ${ }_{2} F_{1}(a, b ; c ; z)$, we get, upon comparison with Eq. (D7),

$$
\alpha=\beta=i \omega_{n},
$$

so the Pöschl-Teller QNM eigenfunctions can be written, in Bizoń-Mach coordinates, as

$$
\phi_{n}^{ \pm}(x)=P_{n}^{\left(i \omega_{n}^{ \pm}, i \omega_{n}^{ \pm}\right)}(x), \quad x \in[-1,1] .
$$

[1] Y. Ashida, Z. Gong, and M. Ueda, Non-Hermitian Physics, Adv. Phys. 69, 3 (2020).

[2] L. N. Trefethen, A. E. Trefethen, S. C. Reddy, and T. A. Driscoll, Hydrodynamic Stability without Eigenvalues, Science 261, 578 (1993).

[3] L. N. Trefethen, Pseudospectra of Linear Operators, SIAM Rev. 39, 383 (1997).

[4] E. B. Davies, Pseudospectra of Differential Operators, J. Oper. Theor. 43, 243 (2000), https://www.jstor.org/stable/ 24715241.

[5] J. Sjöstrand, Pseudospectrum for Differential Operators, Sémin. Équ. Dériv. Partielles 2002-2003, 1 (2003), http:// www.numdam.org/article/SEDP_2002-2003___A16_0 .pdf.

[6] L. Trefethen and M. Embree, Spectra and Pseudospectra: The Behavior of Nonnormal Matrices and Operators (Princeton University Press, Princeton, NJ, 2005).

[7] E. Davies, Linear Operators and Their Spectra, Cambridge Studies in Advanced Mathematics (Cambridge University Press, Cambridge, England, 2007).

[8] D. Krejčiř́ík, P. Siegl, M. Tater, and J. Viola, Pseudospectra in Non-Hermitian Quantum Mechanics, J. Math. Phys. (N.Y.) 56, 103513 (2015).

[9] J. Sjöstrand, Non-Self-Adjoint Differential Operators, Spectral Asymptotics and Random Perturbations. Pseudo-Differential Operators (Springer International Publishing, New York, 2019), https://www.springer.com/gp/ book/9783030108182.

[10] M. Embree and N. Trefethen, Pseudospectra Gateway, https://www.cs.ox.ac.uk/pseudospectra/index.html.

[11] M. J. Colbrook, B. Roman, and A. C. Hansen, How to Compute Spectra with Error Control, Phys. Rev. Lett. 122, 250201 (2019).

[12] S. Chandrasekhar, The Mathematical Theory of Black Holes, in Oxford Classic Texts in the Physical Sciences (Oxford University Press, New York, 2002).

[13] K. D. Kokkotas and B. G. Schmidt, Quasinormal Modes of Stars and Black Holes, Living Rev. Relativity 2, 2 (1999).

[14] H. P. Nollert, Topical Review: Quasinormal Modes: The Characteristic "Sound" of Black Holes and Neutron Stars, Classical Quantum Gravity 16, R159 (1999).

[15] E. Berti, V. Cardoso, and A. O. Starinets, Quasinormal Modes of Black Holes and Black Branes, Classical Quantum Gravity 26, 163001 (2009).

[16] R. A. Konoplya and A. Zhidenko, Quasinormal Modes of Black Holes: From Astrophysics to String Theory, Rev. Mod. Phys. 83, 793 (2011).

[17] E. Berti, V. Cardoso, and C. M. Will, On GravitationalWave Spectroscopy of Massive Black Holes with the Space Interferometer LISA, Phys. Rev. D 73, 064030 (2006).

[18] O. Dreyer, B. J. Kelly, B. Krishnan, L. S. Finn, D. Garrison, and R. Lopez-Aleman, Black Hole Spectroscopy: 
TestingGeneral Relativity through Gravitational Wave Observations, Classical Quantum Gravity 21, 787 (2004).

[19] V. Baibhav, E. Berti, V. Cardoso, and G. Khanna, Black Hole Spectroscopy: Systematic Errors and Ringdown Energy Estimates, Phys. Rev. D 97, 044048 (2018).

[20] M. Isi, M. Giesler, W. M. Farr, M. A. Scheel, and S. A. Teukolsky, Testing the No-Hair Theorem with GW150914, Phys. Rev. Lett. 123, 111102 (2019).

[21] M. Giesler, M. Isi, M. A. Scheel, and S. Teukolsky, Black Hole Ringdown: The Importance of Overtones, Phys. Rev. X 9, 041060 (2019).

[22] M. Cabero, J. Westerweck, C. D. Capano, S. Kumar, A. B. Nielsen, and B. Krishnan, The Next Decade of Black Hole Spectroscopy, Phys. Rev. D 101, 064044 (2020).

[23] E. Maggio, L. Buoninfante, A. Mazumdar, and P. Pani, How Does a Dark Compact Object Ringdown?, Phys. Rev. D 102, 064053 (2020).

[24] J. W. York, Dynamical Origin of Black-Hole Radiance, Phys. Rev. D 28, 2929 (1983).

[25] G. T. Horowitz and V. E. Hubeny, Quasinormal Modes of AdS Black Holes and the Approach to Thermal Equilibrium, Phys. Rev. D 62, 024027 (2000).

[26] C. Barcelo, S. Liberati, and M. Visser, Analogue Gravity, Living Rev. Relativity 8, 12 (2005).

[27] S. V. Dyatlov, Resonances in General Relativity. Ph.D. thesis, UC, Berkeley (2013).

[28] C. M. Warnick, On Quasinormal Modes of Asymptotically Anti-de Sitter Black Holes, Commun. Math. Phys. 333, 959 (2015).

[29] P. Hintz and A.Vasy, Analysis of Linear Waves Near the Cauchy Horizon of Cosmological Black Holes, J. Math. Phys. (N.Y.) 58, 081509 (2017).

[30] H. P. Nollert, Quasinormal Modes of Schwarzschild Black Holes: The Determination of Quasinormal Frequencies with Very Large Imaginary Parts, Phys. Rev. D 47, 5253 (1993).

[31] S. Hod, Bohr's Correspondence Principle and the Area Spectrum of Quantum Black Holes, Phys. Rev. Lett. 81, 4293 (1998).

[32] O. Dreyer, Quasinormal Modes, the Area Spectrum, and Black Hole Entropy, Phys. Rev. Lett. 90, 081301 (2003).

[33] L. Motl, An Analytical Computation of Asymptotic Schwarzschild Quasinormal Frequencies, Adv. Theor. Math. Phys. 6, 1135 (2002).

[34] A. Corichi, On Quasinormal Modes, Black Hole Entropy, and Quantum Geometry, Phys. Rev. D 67, 087502 (2003).

[35] G. Kunstatter, d-Dimensional Black Hole Entropy Spectrum from Quasinormal Modes, Phys. Rev. Lett. 90, 161301 (2003).

[36] L. Motl and A. Neitzke, Asymptotic Black Hole Quasinormal Frequencies, Adv. Theor. Math. Phys. 7, 307 (2003).

[37] M. Domagala and J. Lewandowski, Black Hole Entropy from Quantum Geometry, Classical Quantum Gravity 21, 5233 (2004).

[38] M. Maggiore, Physical Interpretation of the Spectrum of Black Hole Quasinormal Modes, Phys. Rev. Lett. 100, 141301 (2008).

[39] A. J. M. Medved, On the Kerr Quantum Area Spectrum, Classical Quantum Gravity 25, 205014 (2008).
[40] E. C. Vagenas, Area Spectrum of Rotating Black Holes via the New Interpretation of Quasinormal Modes, J. High Energy Phys. 11 (2008) 073.

[41] J. Babb, R. Daghigh, and G. Kunstatter, Highly Damped Quasinormal Modes and the Small Scale Structure of Quantum Corrected Black Hole Exteriors, Phys. Rev. D 84, 084031 (2011).

[42] J. Olmedo and P. Diener (private communication).

[43] S. Carneiro, Quasinormal Modes and Horizon Area Quantisation in Loop Quantum Gravity, arXiv:2012.00227.

[44] H. P. Nollert, About the Significance of Quasinormal Modes of Black Holes, Phys. Rev. D 53, 4397 (1996).

[45] H. P. Nollert and R. H. Price, Quantifying Excitations of Quasinormal Mode Systems, J. Math. Phys. (N.Y.) 40, 980 (1999).

[46] R. G. Daghigh, M. D. Green, and J. C. Morey, Significance of Black Hole Quasinormal Modes: A Closer Look, Phys. Rev. D 101, 104009 (2020).

[47] W. L. Qian, K. Lin, C. Y. Shao, B. Wang, and R. H. Yue, On Asymptotical Quasinormal Mode Spectrum for Piecewise Approximate Effective Potential, Phys. Rev. D 103, 024019 (2021).

[48] P. T. Leung, Y. T. Liu, W. M. Suen, C. Y. Tam, and K. Young, Perturbative Approach to the Quasinormal Modes of Dirty Black Holes, Phys. Rev. D 59, 044034 (1999).

[49] E. Barausse, V. Cardoso, and P. Pani, Can Environmental Effects Spoil Precision Gravitational-Wave Astrophysics?, Phys. Rev. D 89, 104059 (2014).

[50] V. Cardoso, M. Kimura, A. Maselli, E. Berti, C. F. B. Macedo, and R. McManus, Parametrized Black Hole Quasinormal Ringdown: Decoupled Equations for Nonrotating Black Holes, Phys. Rev. D 99, 104077 (2019).

[51] R. McManus, E. Berti, C. F. B. Macedo, M. Kimura, A. Maselli, and V. Cardoso, Parametrized Black Hole Quasinormal Ringdown. II. Coupled Equations and Quadratic Corrections for Nonrotating Black Holes, Phys. Rev. D 100, 044061 (2019).

[52] L. Hui, D. Kabat, and S. S. C. Wong, Quasinormal Modes, Echoes and the Causal Structure of the Green's Function, J. Cosmol. Astropart. Phys. 12 (2019) 020.

[53] G. Khanna and R. H. Price, Black Hole Ringing, Quasinormal Modes, and Light Rings, Phys. Rev. D 95, 081501 (2017).

[54] V. Cardoso, E. Franzin, and P. Pani, Is the GravitationalWave Ringdown a Probe of the Event Horizon?, Phys. Rev. Lett. 116, 171101 (2016); 117, 089902(E) (2016).

[55] R. Konoplya and A. Zhidenko, Wormholes versus Black Holes: Quasinormal Ringing at Early and Late Times, J. Cosmol. Astropart. Phys. 12 (2016) 043.

[56] R. A. Konoplya, Conformal Weyl Gravity via Two Stages of Quasinormal Ringing and Late-Time Behavior, Phys. Rev. D 103, 044033 (2021).

[57] J. L. Jaramillo, R. P. Macedo, and L. Al Sheikh, Gravitational Wave Signatures of Black Hole Quasi-normal Mode Instability, arXiv:2105.03451.

[58] M. Zworski, Resonances in Physics and Geometry, Not. Am. Math. Soc. 46, 319 (1999), https://mathscinet .ams.org/mathscinet-getitem?mr=1668841. 
[59] M. Zworski, Mathematical Study of Scattering Resonances, Bull. Math. Sci. 7, 1 (2017).

[60] S. Dyatlov and M. Zworski, Mathematical Theory of Scattering Resonances, in Graduate Studies in Mathematics (American Mathematical Society, Providence, 2019).

[61] C. Sauvan, J. P. Hugonin, I. Maksymov, and P. Lalanne, Theory of the Spontaneous Optical Emission of Nanosize Photonic and Plasmon Resonators, Phys. Rev. Lett. 110, 237401 (2013).

[62] P. Lalanne, W. Yan, K. Vynck, C. Sauvan, and J. Hugonin, Light Interaction with Photonic and Plasmonic Resonances, Laser Photonics Rev. 12, 1700113 (2018).

[63] R. Penrose, Asymptotic Properties of Fields and SpaceTimes, Phys. Rev. Lett. 10, 66 (1963).

[64] R. Geroch, Asymptotic Structure of Space-Time, edited by E. P. Esposito and L. Witten (Plenum Press, New York, 1976).

[65] A. Ashtekar, Asymptotic Structure of the Gravitational Field at Spatial Infinity, in General Relativity and Gravitation: One Hundred Years after the Birth of Albert Einstein, edited by A. Held (Plenum Press, 1980), Vol. 2, p. 37.

[66] A. Ashtekar, Asymptotic Properties of Isolated Systems: Recent Developments, in General Relativity and Gravitation, edited by B. Bertotti, F. de Felice, and A. Pascolini (Springer Netherlands, 1984), p. 37.

[67] R. M. Wald, General Relativity (Chicago University Press, Chicago, IL, 1984).

[68] H. Friedrich, Conformal Einstein Evolution, in The Conformal Structure of Spacetime: Geometry, Analysis, Numerics, Lecture Notes in Physics, edited by J. Frauendiener and H. Friedrich (Springer, New York, 2002), p. 1.

[69] J. A. V. Kroon, Conformal Methods in General Relativity (Cambridge University Press, Cambridge, England, 2016).

[70] H. Friedrich, On the Existence of $n$-Geodesically Complete or Future Complete Solutions of Einstein's Field Equations with Smooth Asymptotic Structure, Commun. Math. Phys. 107, 587 (1986).

[71] R. P. Macedo, Hyperboloidal Framework for the Kerr Spacetime, Classical Quantum Gravity 37, 065019 (2020).

[72] A. Zenginoglu, A Geometric Framework for Black Hole Perturbations, Phys. Rev. D 83, 127502 (2011).

[73] B. Schmidt, On Relativistic Stellar Oscillations, Gravity Research Foundation essay (1993).

[74] S. Dyatlov, Quasi-Normal Modes and Exponential Energy Decay for the Kerr-de Sitter Black Hole, Commun. Math. Phys. 306, 119 (2011).

[75] M. Ansorg and R. P. Macedo, Spectral Decomposition of Black-Hole Perturbations on Hyperboloidal Slices, Phys. Rev. D 93, 124016 (2016).

[76] R. P. Macedo, J. L. Jaramillo, and M. Ansorg, Hyperboloidal Slicing Approach to Quasi-Normal Mode Expansions: The Reissner-Nordstrom Case, Phys. Rev. D 98, 124005 (2018).

[77] D. Hafner, P. Hintz, and A. Vasy, Linear Stability of Slowly Rotating Kerr Black Holes, arXiv:1906.00860.

[78] D. Gajic and C. Warnick, Quasinormal Modes in Extremal Reissner-Nordström Spacetimes, arXiv:1910.08479.
[79] D. Gajic and C. Warnick, A Model Problem for Quasinormal Ringdown on Asymptotically Flat or Extremal Black Holes, arXiv:1910.08481.

[80] J. Galkowski and M. Zworski, Outgoing Solutions via Gevrey-2 Properties, arXiv:2004.07868.

[81] P. Bizoń, T. Chmaj, and P. Mach, A Toy Model of Hyperboloidal Approach to Quasinormal Modes, Acta Phys. Polon. B 51, 1007 (2020).

[82] M. Maggiore, Gravitational Waves, Astrophysics and Cosmology, Vol. 2 (Oxford University Press, Oxford, 2018).

[83] E. Gasperin and J. L. Jaramillo, Physical Scales and QNM Spectral Instability: The Role of the Scalar Product (to be published).

[84] In the matrix case, $w_{i}^{*} A=\lambda_{i} w_{i}^{*}$, with $w^{*}=\bar{w}^{t}$; i.e., $w_{i}$ are indeed left eigenvectors.

[85] Specifically, we consider "proper eigenvalues" in the sense of belonging to the point spectrum $\sigma_{p}(A)$ of $A$, in particular, not being part of the continuum spectrum $\sigma_{c}(A)$ of the operator. For simplicity, we consider eigenvalues of multiplicity 1 .

[86] T. Kato, Perturbation Theory for Linear Operators (Springer-Verlag, Berlin, 1995).

[87] Still, certain eigenvalues of a non-normal operator (but not all) can have condition number equal to 1 . A "normal eigenvalue" is defined as an eigenvalue $\lambda$ with $\kappa(\lambda)=1$. This notion can be helpful in the study of particular stable eigenvalues in the possibly unstable spectrum of a nonnormal operator.

[88] For the sake of simplicity and clarity, we stay at the matrix level [6]. For a discussion in general Hilbert spaces, cf. Ref. [9].

[89] L. N. Trefethen, Spectral Methods in MATLAB (SIAM: Society for Industrial and Applied Mathematics, Philadelphia, PA, 2000), https://doi.org/10.1137/1.9780898719598.

[90] C. Canuto, M. Hussaini, A. Quarteroni, and T. Zang, Spectral Methods: Fundamentals in Single Domains. Scientific Computation (Springer, Berlin, Heidelberg, 2007).

[91] Also known as the Eckart, Rosen-Morse, Morse-Feshbach potential; see Ref. [92] for a discussion of the terminology.

[92] P. Boonserm and M. Visser, Quasi-Normal Frequencies: Key Analytic Results, J. High Energy Phys. 03 (2011) 073.

[93] V. Ferrari and B. Mashhoon, New Approach to the Quasinormal Modes of a Black Hole, Phys. Rev. D 30, 295 (1984).

[94] H. R. Beyer, On the Completeness of the Quasinormal Modes of the Poschl-Teller Potential, Commun. Math. Phys. 204, 397 (1999).

[95] A. Medved and D. Martin, A Note on Quasinormal Modes: A Tale of Two Treatments, Gen. Relativ. Gravit. 37, 1529 (2005).

[96] J. C. Fabris, M. G. Richarte, and A. Saa, Quasinormal Modes and Self-Adjoint Extensions of the Schroedinger Operator, Phys. Rev. D 103, 045001 (2021).

[97] P. Bizoń and P. Mach, Global Dynamics of a Yang-Mills Field on an Asymptotically Hyperbolic Space, Trans. Am. Math. Soc. 369, 2029 (2017); 369, 3013(E) (2017).

[98] R. Donninger and I. Glogić, Strichartz Estimates for the One-Dimensional Wave Equation, Trans. Am. Math. Soc. 373, 4051 (2020). 
[99] D. Bindel and M. Zworski, Theory and Computation of Resonances in 1D Scattering, http://www.cs.cornell.edu/\% 7Ebindel/cims/resonant1d.

[100] M. Zworski, Distribution of Poles for Scattering on the Real Line, J. Funct. Anal. 73, 277 (1987).

[101] In fact, as far as we are aware of the historical development, the path towards the interest in QNM instability went in the opposite direction: Concerns about BH QNM spectra stability were raised only after modifications or approximations of the potential gave rise to unexpected results [44,45] (Nollert's study itself being motivated by developments in QNMs of leaky optical cavities [102-104], namely, the study of QNM completeness).

[102] P. T. Leung, S. Y. Liu, S. S. Tong, and K. Young, TimeIndependent Perturbation Theory for Quasinormal Modes in Leaky Optical Cavities, Phys. Rev. A 49, 3068 (1994).

[103] P. T. Leung, S. Y. Liu, and K. Young, Completeness and Orthogonality of Quasinormal Modes in Leaky Optical Cavities, Phys. Rev. A 49, 3057 (1994).

[104] E. S. C. Ching, P. T. Leung, W. M. Suen, and K. Young, Quasinormal Mode Expansion for Linearized Waves in Gravitational Systems, Phys. Rev. Lett. 74, 4588 (1995).

[105] More properly and generally [6], one should distinguish the "normal" (indeed, self-adjoint in the particular discussion in the present work) and the "non-normal" operator cases.

[106] Such an operator is relevant by itself since it actually corresponds to the azimuthal mode $m=0$ of a wave propagating on a sphere with a constant unit potential, indeed a conservative system. The eigenfunctions are nothing more than the Legendre polynomials $\phi_{n}(x)=P_{n}(x)$, with real eigenvalues $\omega_{n}^{ \pm}= \pm \sqrt{1+\ell(\ell+1)}$. This provides a robust test case.

[107] Compare this decrease of the error as the numerical resolution increases (the "expected" behavior) with the anomalous growth in Fig. 4, reflecting the fact that the "perturbed operator" has indeed improved spectral stability properties, as compared with the spectrally unstable "unperturbed" Pöschl-Teller operator.

[108] L. Al Sheikh and J. L. Jaramillo, A Geometric Approach to QNMs in Optics: Application to Pseudospectrum and Structural Stability (to be published).

[109] M. Hager, Thesis, Ecole Polytechnique X (2005).

[110] M. Hager, Instabilité Spectrale Semiclassique pour des Opérateurs Non-Autoadjoints. I: Un Modèle, Ann. Fac. Sci. Toulouse Math. 15, 243 (2006).

[111] M. Hager, Instabilité Spectrale Semiclassique d'Opérateurs Non-Autoadjoints. II, Ann. Henri Poincaré 7, 1035 (2006).

[112] M. Hager and J. Sjöstrand, Eigenvalue Asymptotics for Randomly Perturbed Non-Self-Adjoint Operators, arXiv: math/0601381.

[113] W. B. Montrieux, Loi de Weyl presque sûre et résolvante pour des opérateurs non-autoadjoints, Ecole Polytechnique X (2008), https://pastel.archives-ouvertes.fr/pastel00005367.

[114] W. B. Montrieux and J. Sjöstrand, Almost Sure Weyl Asymptotics for Non-Self-Adjoint Elliptic Operators on Compact Manifolds, Annales de la Faculte des Sciences de Toulouse, Mathematiques 19, 567 (2010).
[115] W. B. Montrieux, Almost Sure Weyl Law for a Differential System in Dimension 1, Ann. Henri Poincaré 1, 173 (2011).

[116] W. B. Montrieux, Estimation de Résolvante et Construction de Quasimode Près du Bord du Pseudospectre, arXiv:1301.3102.

[117] M. Vogel, Spectral Statistics of Non-Selfadjoint Operators Subject to Small Random Perturbations, in Séminaire Laurent Schwartz-EDP et Applications (20162017), Exposé no. 19, p. 24, https://doi.org/10.5802/ slsedp.113.

[118] S. Nonnenmacher and M. Vogel, Local Eigenvalue Statistics of One-Dimensional Random Non-Self-Adjoint Pseudo-Differential Operators, arXiv:1711.05850.

[119] J. Sjöstrand, Weyl Law for Semi-Classical Resonances with Randomly Perturbed Potentials, in Mémoires de la Société Mathématique de France 136 (Société Mathématique de France, 2014), https://doi.org/10.24033/ msmf.446.

[120] R. P. Macedo, Comment on Some Exact Quasinormal Frequencies of a Massless Scalar Field in Schwarzschild Spacetime, Phys. Rev. D 99, 088501 (2019).

[121] T. Regge and J. A. Wheeler, Stability of a Schwarzschild Singularity, Phys. Rev. 108, 1063 (1957).

[122] F. J. Zerilli, Gravitational Field of a Particle Falling in a Schwarzschild Geometry Analyzed in Tensor Harmonics, Phys. Rev. D 2, 2141 (1970).

[123] G. B. Cook and M. Zalutskiy, Purely Imaginary Quasinormal Modes of the Kerr Geometry, Classical Quantum Gravity 33, 245008 (2016).

[124] E. Berti (personal website).

[125] V. Cardoso (personal website).

[126] Black Hole Perturbation Toolkit, http://bhptoolkit.org.

[127] L. C. Stein, qnm: A Python Package for Calculating Kerr Quasinormal Modes, Separation Constants, and Spherical-Spheroidal Mixing Coefficients, J. Open Source Softw. 4, 1683 (2019).

[128] K. Lin and W. L. Qian, A Matrix Method for Quasinormal Modes: Schwarzschild Black Holes in Asymptotically Flat and (Anti-) de Sitter Spacetimes, Classical Quantum Gravity 34, 095004 (2017).

[129] A. Jansen, Overdamped Modes in Schwarzschild-de Sitter and a Mathematica Package for the Numerical Computation of Quasinormal Modes, Eur. Phys. J. Plus 132, 546 (2017).

[130] S. Fortuna and I. Vega, Bernstein Spectral Method for Quasinormal Modes and Other Eigenvalue Problems., arXiv:2003.06232.

[131] E. Leaver, An Analytic Representation for the QuasiNormal Modes of Kerr Black Holes, Proc. R. Soc. A 402, 285 (1985).

[132] N. Warburton et al., The Black Hole Perturbation Toolkit (to be published).

[133] P. Bizoń and M. Maliborski, Dynamics at the Threshold for Blowup for Supercritical Wave Equations Outside a Ball, arXiv:1909.01626.

[134] S. Chandrasekhar and S. Detweiler, The Quasi-Normal Modes of the Schwarzschild Black Hole, Proc. R. Soc. A 344, 441 (1975). 
[135] A. Anderson and R. H. Price, Intertwining of the Equations of Black Hole Perturbations., Phys. Rev. D 43, 3147 (1991).

[136] K. Glampedakis, A. D. Johnson, and D. Kennefick, Darboux Transformation in Black Hole Perturbation Theory, Phys. Rev. D 96, 024036 (2017).

[137] M. Dunajski, Solitons, Instantons, and Twistors (Oxford University Press, Oxford, England, 2010).

[138] Such suppression must be stronger than exponential since Pöschl-Teller shows the stability of the fundamental QNM.

[139] The Nollert case $N_{\mathrm{st}}=1$ in his method (iii) corresponds precisely to the cut potential in Sec. VI D 3 and may require a separate discussion. It also connects with Sec. V D 4 since method (iii) in Ref. [44] regularizes Schwarzschild with a Pöschl-Teller factor, cf. Eq. (7) in Ref. [44].

[140] Y. J. Zhang, J. Wu, and P. T. Leung, High-Frequency Behavior of w-Mode Pulsations of Compact Stars, Phys. Rev. D 83, 064012 (2011).

[141] Beyond $w$ modes of compact objects, such perturbed BH universal branches also share features with QNMs of convex obstacles, where the asymptotic form of QNM branches (under a pinched curvature assumption) can be established [58,142] as $\operatorname{Im}\left(\omega_{n}\right) \sim K\left|\operatorname{Re}\left(\omega_{n}\right)\right|^{\frac{1}{3}}+C$, for $n \gg 1$. Focusing on the spherical obstacle case [143] (see also Refs. [59,60]), if considering all angular $\ell$ modes and taking $\ell$ as the spectral parameter (while keeping $n$ fixed), a similar qualitative pattern between the corresponding branches and the perturbed BH QNM branches raises an intriguing question about a possible duality between QNM and Regge poles (cf. e.g., Refs. [144-147] for a complex angular-momentum setting). In particular, the asymptotic logarithm pattern of perturbed-BH [45] and compact object [140] QNMs is exactly recovered for Regge poles of compact objects in Ref. [148] (cf. Ref. [149] for related asymptotics).

[142] J. Sjöstrand and M. Zworski, Asymptotic Distribution of Resonances for Convex Obstacles, Acta Math. 183, 191 (1999).

[143] P. Stefanov, Sharp Upper Bounds on the Number of the Scattering Poles, J. Funct. Anal. 231, 111 (2006).

[144] Y. Decanini, A. Folacci, and B. Raffaelli, Unstable Circular Null Geodesics of Static Spherically Symmetric Black Holes, Regge Poles and Quasinormal Frequencies, Phys. Rev. D 81, 104039 (2010).

[145] Y. Decanini, A. Folacci, and B. Raffaelli, Fine Structure of High-Energy Absorption Cross Sections for Black Holes, Classical Quantum Gravity 28, 175021 (2011).

[146] B. Raffaelli, Strong Gravitational Lensing and Black Hole Quasinormal Modes: Towards a Semiclassical Unified Description, Gen. Relativ. Gravit. 48, 16 (2016).

[147] S. R. Dolan and A. C. Ottewill, On an Expansion Method for Black Hole Quasinormal Modes and Regge Poles, Classical Quantum Gravity 26, 225003 (2009).

[148] M. O. El Hadj, T. Stratton, and S. R. Dolan, Scattering from Compact Objects: Regge Poles and the Complex Angular Momentum Method, Phys. Rev. D 101, 104035 (2020).

[149] T. Daudé and F. Nicoleau, Local Inverse Scattering at a Fixed Energy for Radial Schrödinger Operators and
Localization of the Regge Poles, Ann. Inst. Henri Poincaré 17, 2849 (2015).

[150] We thank B. Raffaelli for pointing this out and also acknowledge Ref. [148].

[151] T. Regge, Analytic Properties of the Scattering Matrix, Il Nuovo Cimento (1955-1965) 8, 671 (1958).

[152] We thank N. Besset for pointing this out.

[153] Such an approach is very much in the spirit of the asymptotic reasoning advocated in Ref. [154], where asymptotic analysis is understood as an efficient and systematic tool to unveil structurally stable patterns underlying universality behavior.

[154] R. W. Batterman, The Devil in the Details: Asymptotic Reasoning in Explanation, Reduction, and Emergence (Oxford University Press, 2006), https://doi.org/10.1093/ 0195146476.001.0001.

[155] M. V. Berry, Semiclassically Weak Reflections Above Analytic and Non-Analytic Potential Barriers, J. Phys. A 15, 3693 (1982).

[156] M. V. Berry and K. E. Mount, Semiclassical Approximations in Wave Mechanics, Rep. Prog. Phys. 35, 315 (1972).

[157] I. Agullo, V. Cardoso, A. del Rio, M. Maggiore, and J. Pullin, Gravitational-Wave Signatures of Quantum Gravity, Phys. Rev. Lett. 126, 041302 (2021).

[158] A. Perez and D. Sudarsky, Dark Energy from Quantum Gravity Discreteness, Phys. Rev. Lett. 122, 221302 (2019).

[159] V. Cardoso, J. a. L. Costa, K. Destounis, P. Hintz, and A. Jansen, Quasinormal Modes and Strong Cosmic Censorship, Phys. Rev. Lett. 120, 031103 (2018).

[160] B. Helffer and J. Sjöstrand, Résonances en Limite SemiClassique, in Mémoires de la Société Mathématique de France, No. 24-25 (Société Mathématique de France, 1986), p. 232, https://doi.org/10.24033/msmf.327.

[161] J. Sjöstrand, Lectures on Resonances, available at https:// sjostrand.perso.math.cnrs.fr/Coursgbg.pdf.

[162] M. Dimassi and J. Sjöstrand, Spectral Asymptotics in the Semi-Classical Limit, London Mathematical Society Lecture Note Series (Cambridge University Press, Cambridge, England, 1999).

[163] N. Dencker, J. Sjöstrand, and M. Zworski, Pseudospectra of Semiclassical (Pseudo-) Differential Operators, Commun. Pure Appl. Math. 57, 384 (2004).

[164] E. B. Davies, Semi-Classical Analysis and PseudoSpectra, J. Diff. Eqs. 216, 153 (2005).

[165] E. B. Davies, Pseudo-Spectra, the Harmonic Oscillator and Complex Resonances, Proc. R. Soc. A 455, 585 (1999).

[166] J. F. Bony, S. Fujiie, T. Ramond, and M. Zerzeri, An Example of Resonance Instability, arXiv:2005.10035.

[167] E. S. C. Ching, P. T. Leung, A. M. van den Brink, W. M. Suen, S. S. Tong, and K. Young, Quasinormal-Mode Expansion for Waves in Open Systems, Rev. Mod. Phys. 70, 1545 (1998).

[168] D. Pook-Kolb, O. Birnholtz, J. L. Jaramillo, B. Krishnan, and E. Schnetter, Horizons in a Binary Black Hole Merger II: Fluxes, Multipole Moments and Stability, arXiv:2006 .03940 .

[169] H. Walther, B. T. H. Varcoe, B. G. Englert, and T. Becker, Cavity Quantum Electrodynamics, Rep. Prog. Phys. 69, 1325 (2006). 
[170] P. J. Schmid, Nonmodal Stability Theory, Annu. Rev. Fluid Mech. 39, 129 (2007).

[171] V. Aldaya, C. Barceló, and J. Jaramillo, Spanish Relativity Meeting (ERE 2010): Gravity as a Crossroad in Physics, J. Phys. Conf. Ser. 314, 011001 (2011).
[172] Note that the resulting associated interpolant $f_{N}(x)$ does not exactly coincide with the $N$-degree polynomial truncation from Eq. (C2) since $c_{k}$ 's in Eq. (C2) are obtained from the orthogonal projection of the exact $f$ on the full Chebyshev complete basis. Both sets of $c_{k}$ 's converge as $N \rightarrow \infty$. 